\title{
RESEARCH IN ELEMENTARY PARTICLE PHYSICS
}

\author{
Progress Report
}

Lai-Him Chan and Richard Haymaker

and

Richard Imlay, Roger McNeil, William Metcalf and Robert Svoboda

\section{Louisiana State Iniversity \\ Baton Rouge, LA}

March 1, 1992 to February 28, 1993

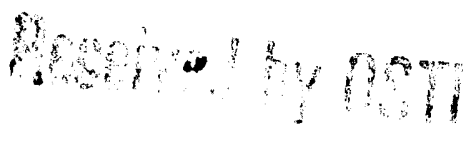

$\therefore \quad 1932$

\section{Prepared for the U.S. Department of Energy under Contract \#DE-FG05-91ER40617}

\section{DISCLAIMER}

\begin{abstract}
This report was prepared as an account of work sponsored by an agency of the United States Government. Neither the United States Government nor any agency thereof, nor any of their employees, makes any warranty, express or implied, or assumes any legal liability or responsibility for the accuracy, completeness, or usefulness of any information, apparatus, product, or process disclosed, or represents that its use would not infringe privately owned rights. Reference herein to any specific commercial product, process, or service by trade name, trademark, manufacturer, or otherwise does not necessarily constitute or imply its endorsement, recommendation, or favoring by the United States Government or any agency thereof. The views and opinions of authors expressed herein do not necessarily state or reflect those of the United States Government or any agency thereof.
\end{abstract}


TABLE OF CONTENTS

Page

I. ABSTRACT . . . . . . . . . . . . . . . . . . 1

II. THEORETICAL - TASK A

A. Low energy particle dynamics and effective field theories, L-H. Chan. . . . . . . . . . . . . . . . . . . 2

B. QCD Dynamics on Lattice, R. Haymaker. . . . . . . . . . . . . 24

C. Lattice Gunge Theory, Ken Yea . . . . . . . . . . . . . . . . 44

III. EXPERIMENTAL - TASKS B and C

A. Introduction. . . . . . . . . . . . . . . . . . . . . 47

B. Neutrino Physics at LAMPF . . . . . . . . . . . . . . . . . 49

C. The ZEUS Experiment at HERA . . . . . . . . . . . . . . 65

D. Non-Accelerator Physics . . . . . . . . . . . . . . 86

E. SSC Activity . . . . . . . . . . . . . . . . . . . 96

F. References .. . . . . . . . . . . . . . 104

IV. REPORTS . . . . . . . . . . . . . . . . . . . . . . 106

Reprints + Prepsints removed 


\section{Estimate of Unobligated Balances}

We do not expect to have a balance at the end of our current year, March 1, 1992 through February 28, 1993. 


\begin{abstract}
We describe theoretical work on effective action expansion on an effective low energy theory of hadrons and lattice gauge theories.

The high energy experimental group at Louisiana State University has analyzed data on a neutrino oscillation experiment at LAMPF. The LSND neutrino experiment is preparing to take data in 1993. IMB data has been analyzed. Preparations for a beam test at KEK for IMB are in progress. Dumand is preparing to test one string of the detector early next summer. The ZEUS electron proton colliding beam experiment has started to take data. Early results have been reported.
\end{abstract}




\section{THEORETICAL - TASK A}

\section{A Low-energy particle dynamics and effective field theories (Lai-Him Chan)}

The emphasis of theoretical particle physics has been centered on higher energy and shorter distance where the dynamics of particles and fields is presumably more fundamental and perhaps simpler. However particle phenomenology as observed in laboratories occurs at low energy. In many cases there is no first principle method of calculation to relate theories directly and experimental observations. Therefore it is vitally important to develop theoretical tools and conjectures which serve as intermediate stepping stones for the construction of an effective theory that would eventually lead the ultimate understanding of particle physics at the fundamental level.

In the past years I have pursued this endeavor successfully in a number of ways as will be described in more detail in the following sections. My general approaches are:

1. to use a set of acceptable principles or scenarios together with model-independent assumptions, which are either derivable or believed to be derivable from a more fundamental theory, to extrapolate from existing data predictions which may be useful either as relevant information for further data analysis or as a theoretical check on the validity of the general assumptions. The example for this approach is my work on the isospin mass splittings $[1,2,3]$.

2. to extract frum experimental observations some general properties or structures of the dynamics which may not be anticipated directly from the fundamental interactions. The recognition of such principles or structures may give insight to the ultimate solution to the dynamical problem. The example of my work in this direction is my conjecture on the scalar quark confinement which have been finally confirmed theoretically and experimentally only very recently after many years [13]. 
3. to develop mathematical tools which may be used to extract from a fundamental theory at shorter distance an effective field theory for low-energy dynarnics. A series of my recent papers on effective action expansions contributes toward this end $[5,6,7,8,9,10]$.

4. to develop an effective field theory for the low-energy hadron dynamics using effective action expansion and assumptions from QCD $[6,8]$.

In the following pages, I shall first describe some major innovation in mathematical tool in doing theoretical physics at LSU, Mathematica, a powerful symbolic manipulation computer program. Then I shall report the progress and the future direction on a few research programs.

\section{A.1 Symbolic manipulation programming}

In the last few decays, through the success of the standard model, theoretical particle physics has been transformed into a mature field of science. The important symptom is the slowness in making any significant progress, which causes a great deal of frustration among particle theorists. The reason of this has been mostly attributed to the lack of new and exciting experimental results. However more importantly in my own opinion, it is caused by the enormous amount of work in order to translate any interesting theoretical idea into a useful form ready to be tested. More seriously one is also facing a big risk that the hard work would be wasted if his idea may not necessarily work out.

With recent development of economically priced fast cpu workstation with large memory capacity, symbolic manipulation computation can very well provide the thrust desperately needed in particle theoretical physics. Various symbolic manipulation programs have been used for well-defined but tedious chores suck as calculations of Dirac Gamma matrices, Feymann's diagrams, scattering cross sections etc. but not for creative analytical works. If theoretical particle physics is to remain a viable intellectual science competitive to attract next generation talents, tremendous effort must be made to use symbolic manipulation computation as a tool to provide an entirely integrated environment for doing theoretical physics in much 
the same way as desktop computers with word processing, spreadsheet and other softwares replacing typewriters and adding machines to provide an modern office environment.

A symbolic manipulation Language, Mathematica,developed commerci.lly by former particle theorist Stephen Wolfram would furnish an ideal basis for such purpose. He is dedicated and committed to continuously upgrade his product with the goal for a total environment of doing mathematics. More importantly Mathematica has been a commercial success with would more or less guarantee continuous future support and development. However most of the packages of Mathematica are general purpose softwares. In order to use Mathematica to do creative research in particle physics and field theory, it is necessary to take the leadership to invest on the development of new tools and techniques, which can be very time consuming.

Most of previous use of symbolic manipulation languages has been concentrating on procedure programing for doing many time-consuming tedious chores. However the real power of Mathematica lies on its use in functional programming which has become a very exciting area in high-level computer language. In functional program one can implement algorithms directly.

The process of reorientation to this new environment of solving problems has been slow because the logic are very much different from the intuition we acquired through many years of experience and the software itself has been constantly upgraded. However the reward of the investment may be worthy of such effort. We shall briefly summarized our progress here.

The calculation of the derivative expansion is an excellent example of what symbolic manipulation programming can accomplish. Derivative expansion is an expansion of a nonlocal expression into an infinite series of local expressions. Effective action expansion is an example of the derivative expansion in quantum field theory. The effective potential is the leading term of this expansion. It was first introduced by Coleman and Weinberg and the calculation was substantially simplified by Jackiw. The two-derivative term for the $\phi^{4}$ model was later calculated by Illiopoulos and Itzykson in an very obscure way. The method was hard to follow and cannot be generalized for the calculation of higher-derivative terms. Not until ten 
years later the four-derivaîive terms were calculated. Using the background field technique I have proposed a very systematical and natural method to generate all higher-derivative terms. However the tensor algebra becomes more tedious as the number of terms increases very rapidly as the number of derivatives goes up. It is very obvious that it is not practical to do the computation by hand. The calculation of the higher-derivative terms is very important because unlike the ordinary expansion series, this functional series has never been studies mathematically. We do not know that any definite pattern for the higher-derivative terms. There is no natural expansion parameter. The convergency property has not been investigated before. There is no hint on how to analytical continue the series in order to extend the domain of the series. These knowledge is important in order that the derivative expansion can become truly useful for meaning applications in physics. The only way to achieve this is to calculate more terms in this series. Mathematica would be a natural environment to do this.

Since the calculations would involve substantial amount of tensor manipulation, it is necessary to have a program to handle tensor algebra efficiently. In particular the program must recognize not only the dummy summation notation but also the equivalence terms with different dummy indices. It is very difficult to find efficient algorithms to implement such capability. The program must also carry out tensor differentiation. In addition it should be general enough to treat operator or matrix tensors and nonabelian algebra as well as recognizing the trace properties. It is clear that no existing softwares would be sophisticate enough to do this job. After many trials finally I found a acceptable algorithms to implements all these criteria's. The program is listed in the next two pages for reference. The program has bern fully tested for abelean algebra and partially tested for nonabealen algebra, meaning that there can be still bugs in certain exceptional cases. The program is functional but cas be further polished for efficiency.

The calculation of the effective action expansion in one-loop quantum correction in most cases can be reduced to calculate the derivative expansion of $\operatorname{det}\left[\partial^{2}+U(x)\right]$, which procedure has been discussed in detail in Ref. In the nonabelian case, the function $U(x)$ may be a matrix in the internal symmetry space or in Dirac Gamma 
matrix. For the initial step, I specialize to the abelean case. The program is only one page long and is listed after the tensor program. The nontrivial part of the program is to simplify the equivalent terms by performating integration by parts to reduce the result to a minimum number of canonical indepenent terms. The final calculation is summarized in the page following the program. The entire calculation of the derivative expansion up to the $\mathrm{n}$-derivative term can be called by a single function DExpansion[n] and the complete computation from the beginning to the final step is carried out by the NeXt workstation without any interference of human being. The 2- and 4-derivative terms are identical to the published results and the 6 - and 8-derivative terms are completely new. The computing time is also shown. It takes $4.5 \mathrm{sec}, 1.5 \mathrm{~min}, .5 \mathrm{hr}$ and $9 \mathrm{hrs}$ to compute the 2-, 4-, 6- and 8-derivative terms respectively. Each successive higher derivative terms require approximately twenty-fold more computer time to complete, although the number of terms only goes up three fold each time. If faster computer is avaiable, the same program can be used to calculate 10 - and other higher derivative terms.

In the case of one dimension, cumbersome tensor algebra is not necessary. The computer time to compute each successive higher derivative terms increases only three-fold instead of twenty-fold. It becomes possible to compute the derivative expansion up to 22-derivative terms. Certain patterns can be extracted from this complicated functional series. At least some terms can be written as a well-defined infinite series and my speculation is that with some more work it may be possible to write the general form of the infinite functional series. In addition I have also found a new method to generate a new series from the derivative expansion series such that the new series has a definite expansion parameter and can be continued analytically. I have applied this method successfuly to calculate the quantum correction of the soliton where the normal derivative expansion is not only divergent but each derivative term actually becomes infinite within the range of integration.

In the near future I will extend this calculation to include the nonabelian algebra which would be extremely useful for some of the applications describe below. Some definite progress has been made in those direction, but within the limitation of space, I shall omitted the details here. 


\section{TENSOR}

\section{Metric}

g/:Attributes $[\sigma]=0 x d e r l e n s$;

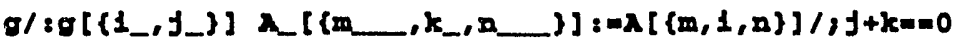

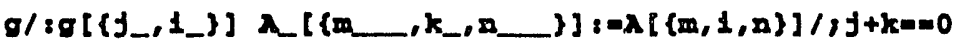

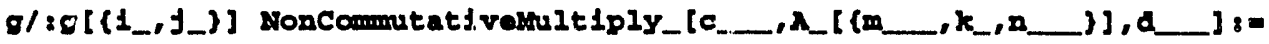
NonCommutat ivesultiply $[c, \lambda[(m, i, n)], d] /, j+k=0$

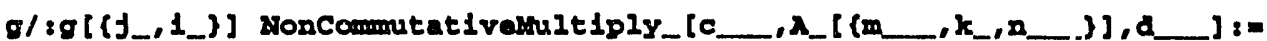
NonCommutat I vamit Iply $[c, \lambda[(m, 1, n)], d] /, j+k==0$

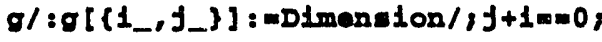

apeilon/:apeilon $\left.\left[\left\{\ldots, a_{-}, \ldots,-a_{-}, \ldots\right]\right\}\right]:=0$

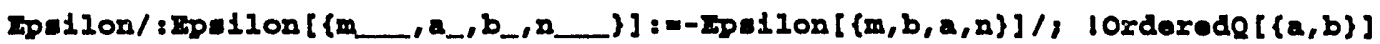

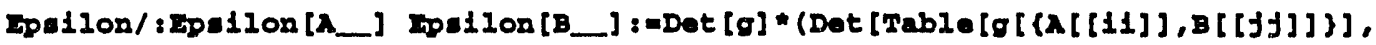

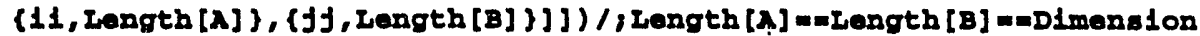

\section{- Rules for Noncommatative Multiplication}

Unprotect [NonComutat IVoultiply];

Clear [NonCommutat 1 vesult Iply]

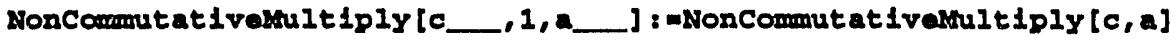

NonComutat 1 romaltiply $[1]=1$;

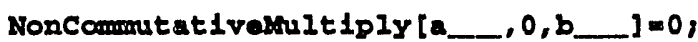

rormat [NonCommutat 1vakultiply [a__ ] ] : sequencerorm [ [",a, " ] "]

Protect [NonComoutativamitiply];

\section{Format}

( (Format $\left[\#\left[\left\{1 \_\right\}\right]\right]$: = SequenceForm [\#, Sequence of Thread $[$Index $\left.\left.[\{1\}]]\right]\right)$.

[1ndex [x_8ymbol] ->Superacript $[x]\} /$.

Index [x_string] - > Superacript [x]/. Index [x_Ttmes] - >Subscript $[-x] /$. Index [x_Integex] $\rightarrow$ Subecript $[x] /$. Index $[" D "]->(p] *) \& / \theta(0, \sigma, D))$;

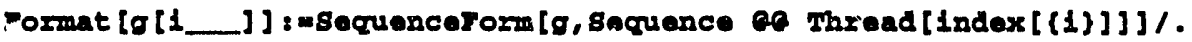

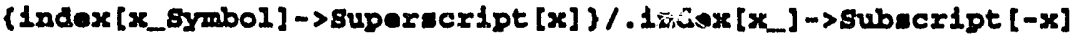

\section{Matching Dumray indices}

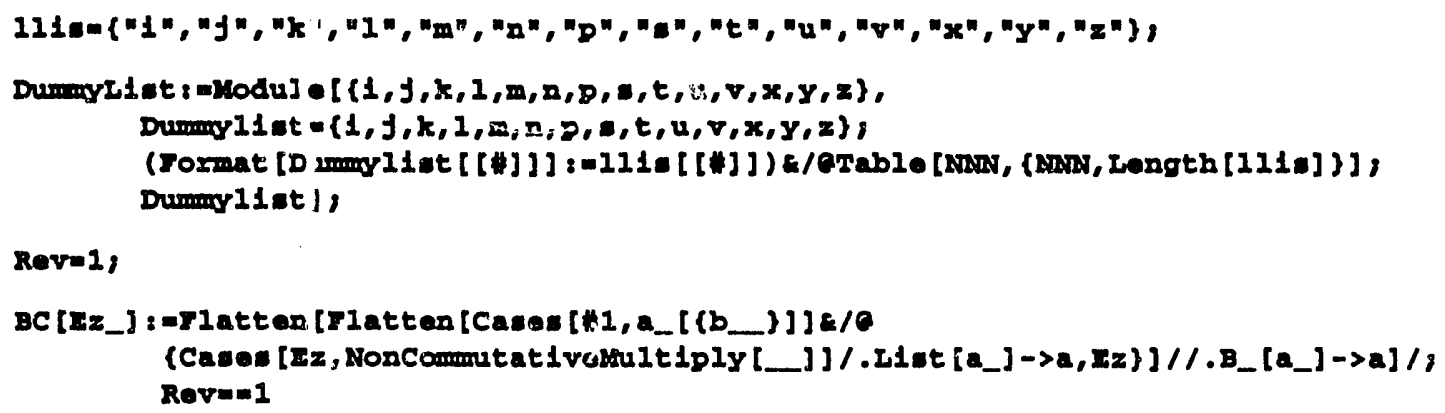




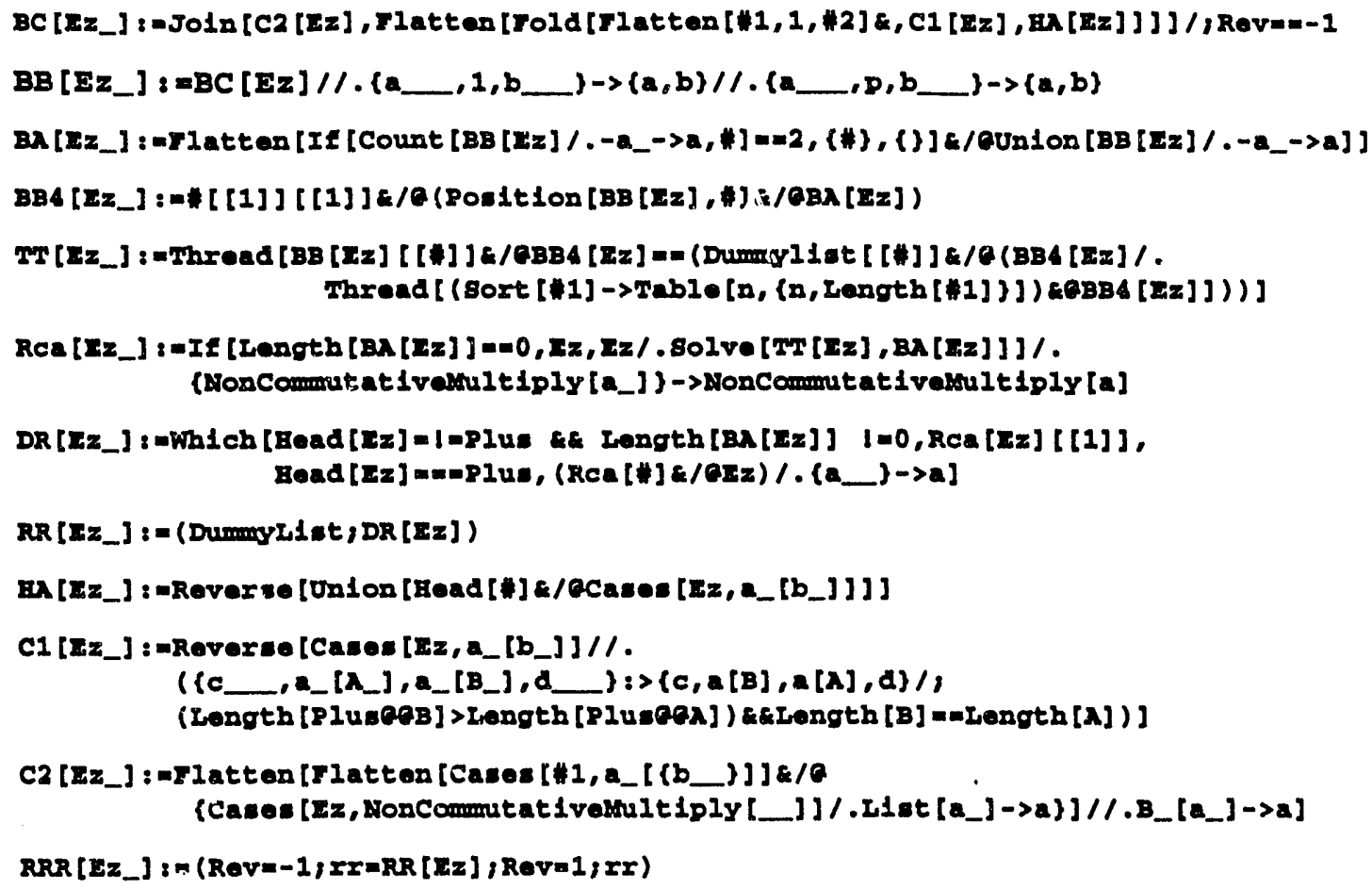

\section{Trace}

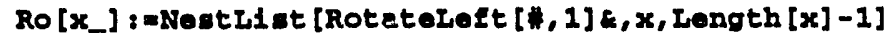

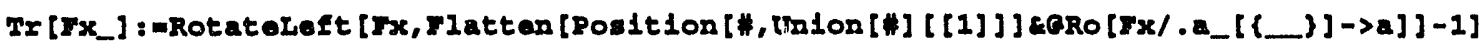

$T x\left[x_{-}+y_{-}\right]:=T x[x]+T x[y]$

$\operatorname{Tr}\left[a-x_{-}\right]:=(a \operatorname{Tr}[x] / 8$ Bead $[a]-1=$ NonCommutat 1 vaMultiply)

\section{Tensor Differentiation}

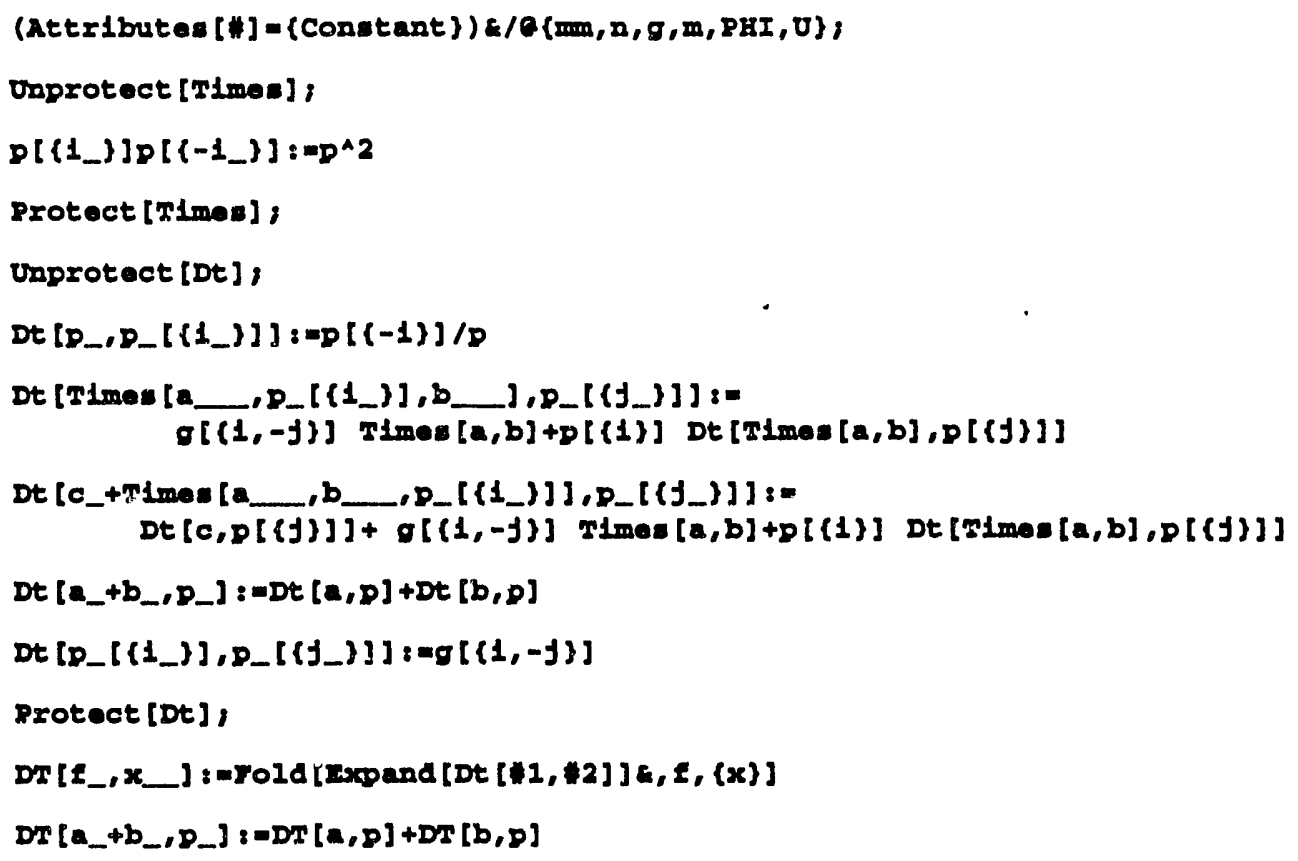




\section{Derivative Expansion of $\operatorname{Det}_{\left[d^{\wedge} 2+U[x]\right]}$}

\section{Expansion}

\$Recuralondimit $=\$$ Iterat londimit -1000

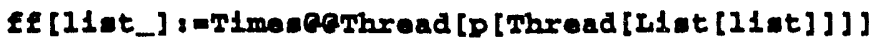

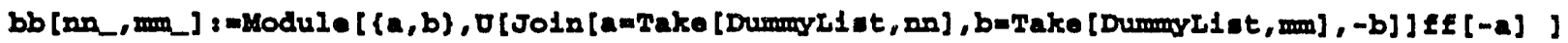

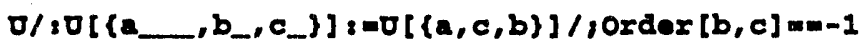

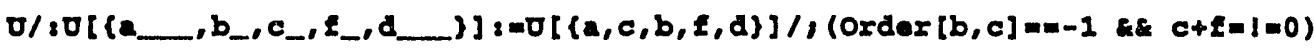

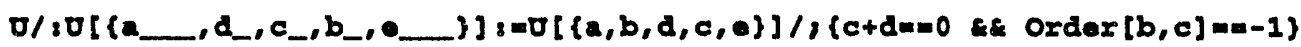

$\sigma /: 0\left[\left\{a_{2}, a_{-}, c_{-}, b_{-}\right\}\right],=\sigma\left[\left(a, b, d_{,}, c\right)\right] /,(c+d==0)$

$G[p, n n]:=\operatorname{Col} 1 e c t\left[\operatorname{sum}\left[\right.\right.$ Gama $[n-1+1] /(\operatorname{Gamma}[n-21+1]$ Gama $[1+1])(-2 I)^{\wedge}(n-21)$ bb $[n-21,1] *$ $\triangle \wedge n /\left(p^{\wedge} 2+m^{\wedge} 2\right)^{\wedge}(n+2-1),\{n, 1, n n\},(1,0$, F100r $\left.\left.[n / 2]\}\right] / / R R, n\right]$

Pr $[0$, nn_ $]:=\mathrm{Expand}[$ Module $[\mathrm{fa}\}$,

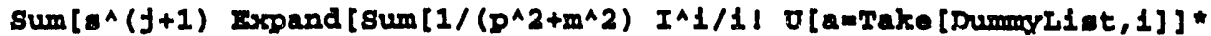

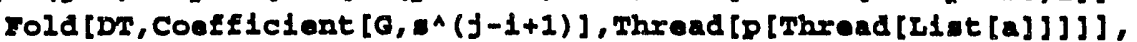

$(1,1, j\}]\},\{f, 1, n R-1\}]\}] / / R R / / R R R$

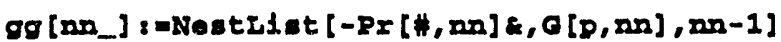

Momentum Integration

Jl1 at $=(a x, b x, c x, d x, a x, \pm x, g x, b x, 1 x, y x, b x, 1 x)$,

JAI1 $=t=\left\{a x_{-}, b x_{-}, c x_{-}, d x_{-}, e x_{-}, t x_{-}, g x_{-}, b x_{-}, 1 x_{-}, j x_{-}, k x_{-}, I x_{-}\right\}$,

Format $[a x]-a$, Format $[b x]=b$, rormat $[c x]=c$, rormat $[d x]=d$, rormat $[0 x]=0$, rormat $[\& x]=f$, rormat $[g x]=g$, Format $[b x]=b$, rormat $[1 x]-1$, rormat $[j x]=f$, rormat $[k x]=k$, rormat $[1 x]=1$,

Attributes [Joc] $-\{$ Orderloss\},

$\operatorname{Jec}\left[\left(a_{-}, b_{-}\right\}\right]:=g[\{a, b\}]$

$\operatorname{Jec}\left[\left\{a, b \_\right\}\right],=\operatorname{Rrgand}[\operatorname{Sum}[g[\{a,(b)[[a]]\}] \operatorname{Jec}[\operatorname{Drop}[\{b\},\{a\}]],\{a$, Length $[\{b\}]\}]] /$, Length $[(b)]>1$

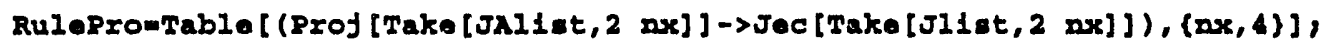

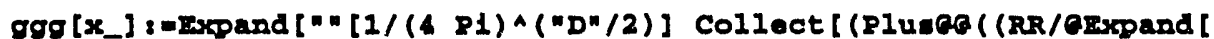

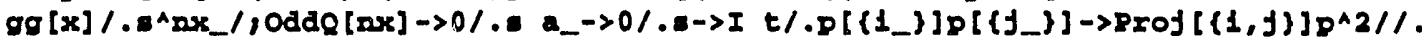

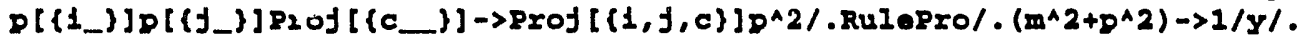

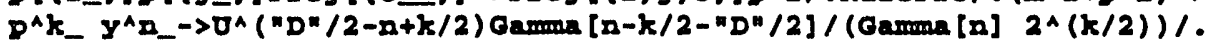
$Y^{\wedge} n_{-} \rightarrow \sigma^{\wedge}\left(" D^{n} / 2-n\right)$ Gamma $\left[n-" D^{n} / 2\right]$ /Carma $\left.\left.\left.\left.\left.\left.[n]\right]\right)\right)\right), t\right], t\right]$

Simplification throught Integration by Part

II [ex_] : mCases [Leve] [\#, $\{1\}], 0[\ldots]$ ]

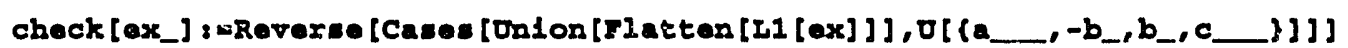

term [ex_] inlatten [Position [ex, \#] \&/Ocheck [ex] , 1] [ [1]]

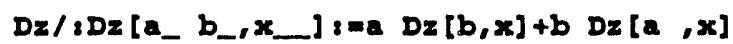

$D z / 8 D=\left[a_{-} b_{-}, x_{-}\right]$ina $D z[b, x] /, F x \bullet 0 Q[a, 0]$

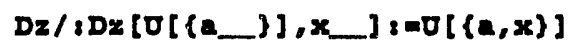

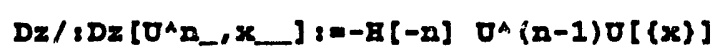

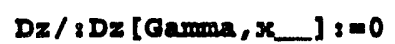

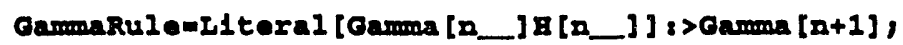

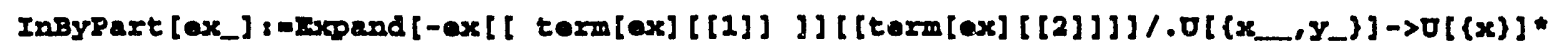
$D z[$ Drop[ex[[term[ox] [[1]] ]\}, fterm[ex] [[2]]\}],

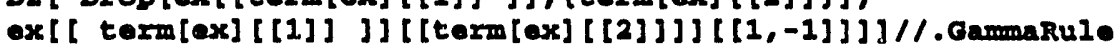

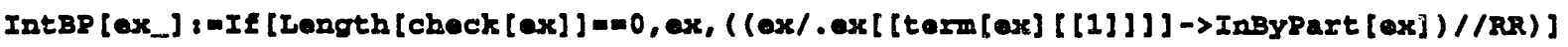

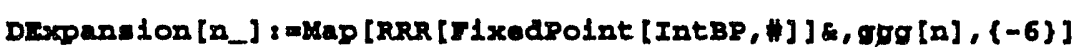


(4.56667 second, $\left.\frac{-\left(t^{2} v^{-4}+D / 2\left(\frac{1}{Q^{D / 2} P i^{D / 2}}\right] \text { Gamanal }\left(4-\frac{D}{2}\right] v_{i} v^{i}\right)}{22}\right)$

Timing [DExpaneion [4]]

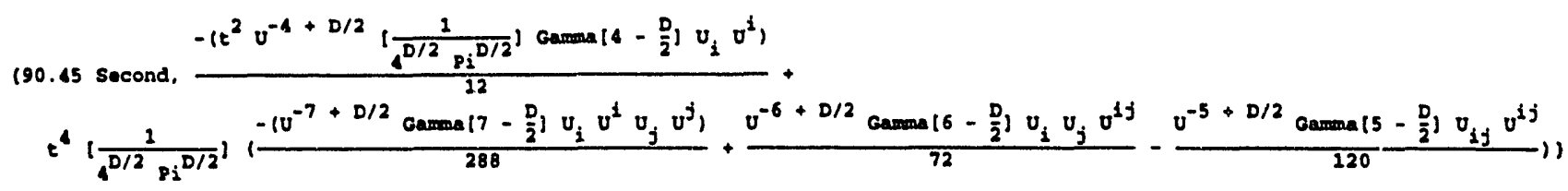

\section{Finfing (DExpanston (6)]}

$$
\begin{aligned}
& \frac{-\left(t^{2} v^{-4}+D / 2\left(\frac{1}{D / 2} P_{i}^{D / 2}\right) \text { Gamal }\left(4-\frac{D}{2}\right) v_{i} v^{i}\right)}{12}+
\end{aligned}
$$

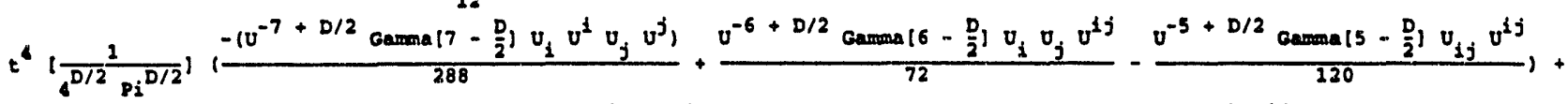

$$
\begin{aligned}
& e^{6}\left(\frac{1}{4^{D / 2} p_{i}^{D / 2}}\right)\left(\frac{-\left(U^{-10+D / 2} \operatorname{Gamma}\left(10-\frac{D}{2}\right) v_{i} v^{i} u_{j} v^{j} v_{k} v^{k}\right)}{10368}+\frac{u^{-9+D / 2} \operatorname{Gamma}\left(9-\frac{D}{2}\right) v_{i} v_{j} v_{k} v^{k} v^{1 j}}{866}\right. \text {. }
\end{aligned}
$$

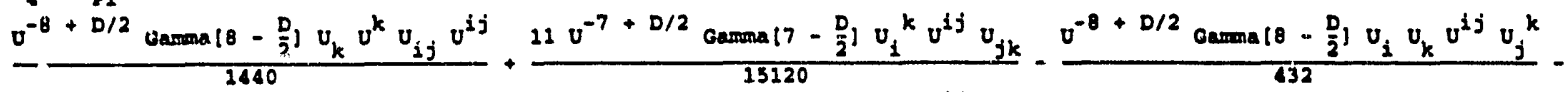

$$
\begin{aligned}
& \left.\frac{v^{-8}+D / 2 \operatorname{Gammo}\left(8-\frac{D}{2}\right) u_{i} v_{j} v_{k} u^{i j k}}{1296}+\frac{u^{-7}+D / 2 \operatorname{Gamma}\left(7-\frac{D}{2}\right) u_{i} u_{j k} u^{i j k}}{360}-\frac{\left.u^{-6}+D / 2 \text { Garmai6 }-\frac{D}{2}\right) v_{i j k} u^{i j k}}{1680}\right)
\end{aligned}
$$

TIming [DExpansion (B)]

$$
\begin{aligned}
& \left\{31877.5 \text { second, } \frac{-\left(t^{2} v^{-4}+D / 2\left(\frac{1}{Q^{D / 2} 8 i^{D / 2}}\right) \text { Oamma }\left(4 \cdot \frac{D}{2}\right) v_{i} v^{1}\right)}{12}+\right. \\
& t^{4}\left(\frac{1}{D / 2} P_{i}^{D / 2}\right)\left(\frac{-\left(u^{-7}+D / 2 \operatorname{Garma}\left(7-\frac{D}{2}\right) v_{i} v^{i} v_{j} v^{j}\right)}{288}+\frac{v^{-6}+D / 2 \operatorname{Gamma}\left(6-\frac{D}{2}\right) v_{i} v_{j} v^{i j}}{72}-\right. \\
& \left.\frac{\mathrm{U}^{-5}+D / 2 \text { Gamens }\left(5-\frac{D}{2}\right) v_{i j} v^{i f}}{120}\right)+t^{6}\left(\frac{2}{i^{D / 2} P i^{D / 2}}\right)
\end{aligned}
$$

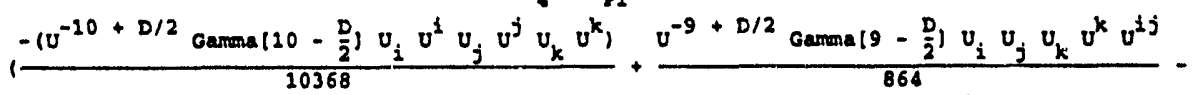

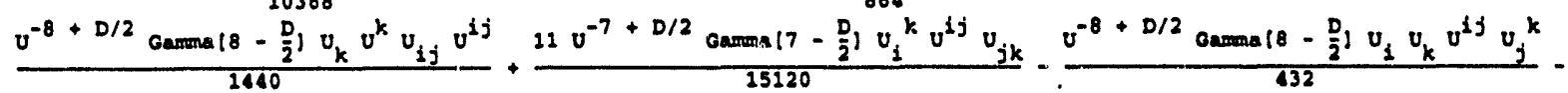

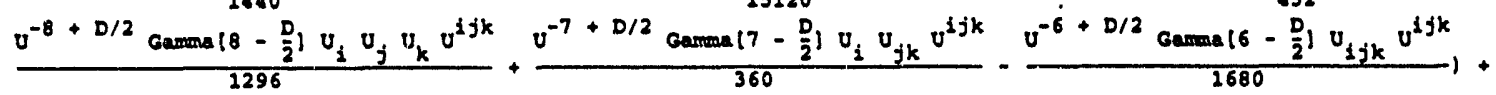

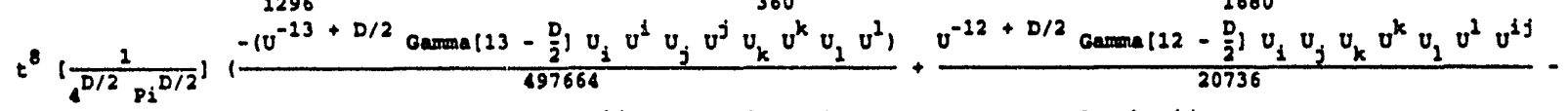

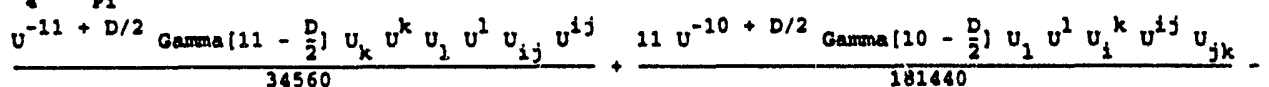

$$
\begin{aligned}
& \frac{v^{-11+D / 2} \text { Gamal11 - } \frac{D}{2} ! v_{i} v_{k} v_{2} v^{1} v^{i j} v_{j}^{k}}{5184}+\frac{\left.v^{-10+D / 2} \text { Gammal10- } \frac{D}{2}\right) v_{k} v_{1} v_{i}^{2} v^{1 j} v_{j}^{k}}{2592}-
\end{aligned}
$$

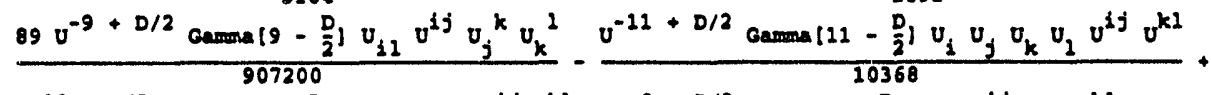

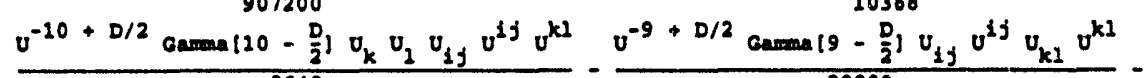

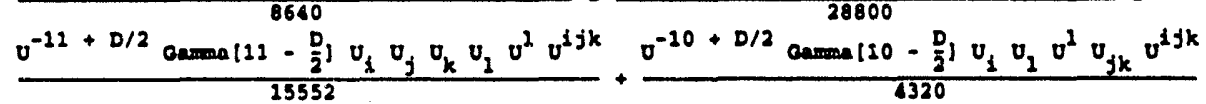

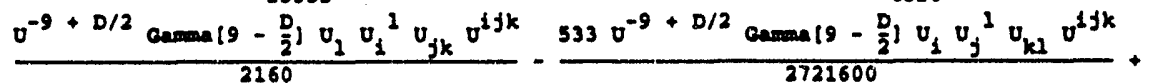

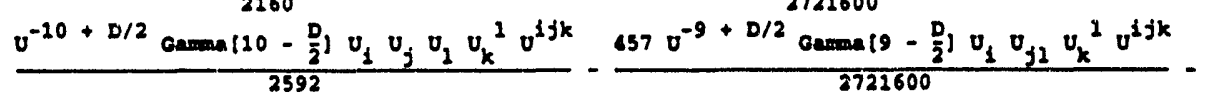

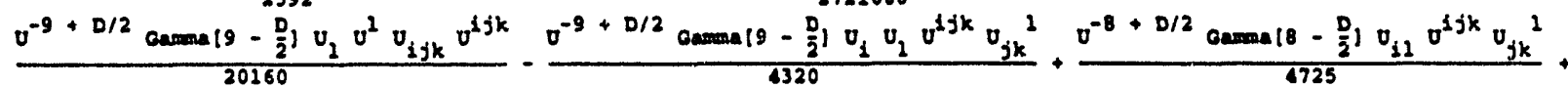

$$
\begin{aligned}
& \frac{u^{-10+D / 2} \operatorname{Gama}\left(10-\frac{D}{2}\right) v_{1} v_{f} v_{k} \delta_{1} v^{1 j k 1}}{31108}-\frac{0^{-9}+D / 2 \operatorname{comal}\left(9-\frac{D^{2}}{2}\right) v_{i} v_{j} v_{k 1} v^{i j k 1}}{4320}+
\end{aligned}
$$

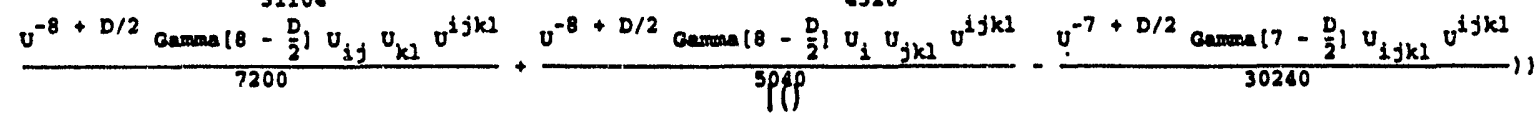




\section{A.2 Derivative expansion and effective action expansion}

Much progress and speculations in fundamental particles interactions in the recent years have based on hierarchy of levels of effective dynamics. While the fundamental field theories may appear to be simple and perhaps elegant at very short distance, the connection to the observable dynamics at low energy level are extremely complex and remote. With the emerging problems such as the Skyrmion physics, strongly interacting Higgs sector of the standard model, supersymmetric nonlinear $\sigma$ model, supergravity, anomalies etc., there has been increasing interest toward effective field theories with higher derivative couplings. Important progresses have been achieved for the efficient derivation of the derivative (effective action) expansions by integrating out the non-observable quantum degrees of freedom associated with the short distant dynamics $[5,6,7,8,9,14,15,16,17,29]$. In particular my method has been followed by works from major groups such as Berkeley $[16,17,22,23,24,25,26,27,28]$ and Seattle Washington $[30,31,32]$, in diversified areas from nuclear and soliton physic: to supersymmetry and supergravity. This new tool opens up many applications as well as many questions which has not been raised before. In addition there may be also improvements on the technique and generalizations to more complex and realistic problem such as the non-abelian-gauge-meson-loop.

An important aspect of my formulation is that the derivative expansion can be developed systematically in a straightforward manner without having to solve any equation. I have used this method to calculate the four derivative terms in a number of models. In a number of problems it is clear that it may be useful to go beyond the four-derivative terms. However the algebra for the computation becomes unmanageable. With the help of Mathematica, hopefully this task can be accomplished.

I am going to describe only a few examples in this proposal my research in this direction. 


\section{A.2.1 Improvement of the derivative expansion and new direction}

In spite of its many possible applications, the use the derivative expansion is restrictive because the series does not converge or not converge fast enough in some interesting cases. Important examples are vacuum energy in the presence of background fields and quantum correction to the soliton energy. Several attempts have been directed toward finding a possible solution or partial solution to this problem.[10][30,?,32] The latest work by Wasson and Koonin [21] is an very interesting effort to patch up what derivative expansion fails to accomplish by a brute force numerical calculation. In the process of formulating a smooth transition between the two process, they have actually provided some important hint which stimulates my present work.

It is interesting to point out that the derivative expansion is the equivalence of WKB expansion. Given a potential $\mathrm{U}(\mathrm{x})$ such that $U(\infty)=U(-\infty)=$ Constant and $U(x)$ is a smooth function of $x$. The WKB expansion for a physical quantity such as the phaseshift at a given energy $E$ is in fact a derivative expansion in powers of the derivative of $U(x)$. The dimension of the higher derivatives is balanced by an appropriate inverse factors of $\sqrt{E-U(x)}$. It is clear that (1) the WKB expansion is a high energy expansion, (2)it is not possible to extract bound states information from the WKB approximation, and (3) there is no unique expansion parameter in the derivative expansion and therefore it becomes impossible to construct any sensible analytical continuation scheme for the series. To resolve these difficulties I have constructed another high energy approximation, a power series expansion in the inverse power of momentum $p=\sqrt{E-U(\infty)}$ with coefficients given by some combination of $U(x)-U(\infty)$ and derivatives of $U(x)$, or equivalently an expansion of small $U(x)-U(\infty)$ and small derivatives of $U(x)$ (or derivatives of $U(x)-U(\infty)$ since $U(\infty)$ is a constant). I shall refer this expansion as the large loop-momentum expansion. The new series has much better behavior. In addition, one can use the well known methods such as Padé approximant or Borel summation to analytically continue the series beyond its radius of convergence. More importantly the analytical properties of potential scattering is well known in the complex momentum plane and they can be used as powerful constraints in constructing the analytic continuation. 
This method can readily be applied to the derivative expansion in quantum field theory. It is of particular interest to use it to calculate the vacuum energy of a particular backgro nd field configuration because the conventional derivative expansion fails to converge in many instances. Quantum correction to the soliton mass is a special example. In the background field formulation of the effective action derivative expansion, the quantum loop correction is performed in momentum space while the effective action is expressed automatically in configuration space. The energy for a static backgrounfield can be obtained by calculating the effective action without the time integration. If one reserves the loop-energy integration to be performed at the very end, one can immediately recognize that the convergence of the large momentum expansion is controlled by the singularities in the complex momentum plane closest to zero energy, namely the ground state and other lowlying poles. For a soliton backgroud field, the ground state is the zero energy mode from the translational invariance and therefore it can be folded into the series to yield a much more convergent series. For the Sine-Gordon soliton, there is no other singularity and the series terminates to yield the exact solution. In the case of the one-dimension $\phi^{4}$ solition, it reduces to a geometric series convergent everywhere and can be summed up to give the exact pole location and therefore the exact solution. To check how this method works for more interesting case of $\phi^{4}$ theory coupled to a fermion field with Yukawa coupling g. Figure 1 shows the convergence of the expansion series for the fermion loop energy in the background classical soliton field, in comparision to the exact result.

A slight variation of this technique has been applied to a non-soliton static background fields,

$$
\phi(x)=1-\frac{\phi_{b}}{1+\exp \left[\left(x^{2}-R^{2}\right) / T^{2}\right]}
$$

, in the one-dimensional $\phi^{4}$ theory and the results for the vacuum energy agree well with those calculated by Wasson and Koonig [21] and also Li,Perry and Wilets [30] with a wide varieties of parameters $R, T$ and $\phi_{b}$. The agreement becomes worse for small $\mathrm{T}$ as it is expected since the potential gradually turns into a square well with sharp discontinuity.

This method can be readily generalized to higher dimension, more complicated 
field theories including fermions and gauge fields as well as higher order calculation with multi-loop contribution.

There is one very important result coming out from this calculation rather unexpectedly but it may have much wider implication. The calculation show that the simplification actually is derived from the natural expansion of $\phi(x)$ and $U[\phi(x)]$ around $x \rightarrow \infty$ which is the asymptotic classical ground state which may or may not be a symmetric vacuum. From dimension analysis such expansion naturally isolates the divergences in the decreasing order and renormaliztion can be performed almost automatically. In the conventional approach one defines the renormalization for the symietry thecsy and analytically continues the parameters of the symmetrical theory to the spontaneous symmetry breaking limit, whereas the present formulation asserts that it is simpler and it also gives a better approximation when the renormalization is performed ai the natural vacuum. This procedure makes the higher order calculation much easier. A immediate consequence is that there will be finite renormalization for the wave-function renormalization as well as other finite renormalizations for the $\phi^{4}$ theory not required by symmetric renormalization.

\section{A.2.2 Effective action expansion for the nonlinear $\sigma$ model - the nor- mal coordinate method}

I have proposed to investigate whether the $S O(N)$ nonlinear $\sigma$ model is equivalent to the $m_{\sigma} \rightarrow \infty$ limit of the linear $\sigma$ model by comparing the corresponding one-loop effective action expansions up to the 4-derivative terms and including the symmetry breaking term [9].

For this purpose I use a new background field method to calculate the effective action expansion directly. In the case of the linear $\sigma$ model the renormalization procedure is implemented carefully before the $m_{\sigma} \rightarrow \infty$ limit is taken. For the nonlinear $\sigma$ model I introduce a new and intuitive covariant treatment for the perturbation calculation of the field theory with nonlinear constraint. I do not find any

noninvariant terms in either case $[41,42,43,44]$. I show that the divergent parts of the effective Lagrangians due to $m_{\pi} \rightarrow 0, m_{\sigma} \rightarrow \infty$, or $N \rightarrow \infty$ are equivalent in 
the two models. However the nonleading finite parts of the effective Lagrangians are different. Therefore the two operations, taking the $m_{\sigma} \rightarrow \infty$ limit and calculating the quantum corrcations, do not commute. The origin of this difference may be a violation of decoupling.

To make sure that my new method for calculating the effective Lagrangian for the nonlinear $\sigma$ model is reliable, I have also carried out the corresponding calculation using the normal coordinate method of Ref. [45] and obtained the same result[46]. It is interesting that one can actually identify the corresponding expressions and contributions such as those in Eq. (6.28) between the two methods.

However there may be some subtle difficulties not anticipated in the construction of the effe tive action for the nonlinear $\sigma$ model due to the constraint, such that Eq.(6.30) may not be valid even to the one-loop order Lagrangian.[47] The investigation of this aspect is presently in progress.

Fin the time being, the linear $\sigma$ model and the nonlinear $\sigma$ model are the only viable models to provide the spontaneous symmetry breaking urgently needed to understand any unified picture in particle physics. Even with the limited scope of applicability, our new method and analysis have taken a new small step toward the understanding of this fundamental problem.

\section{A.2.3 Pffective Lagrangians from chiral quark dynamics}

The Skyrme model has been very successful in unifying the low-energy hadron physics [39]. However it can only be considered as a phenomenological field theory. Ultimately the Skyrmion Lagrangian must be an effective Lagrangian derivable from a more fundamental Lagrangian valid at a shorter distant scale. Ideally it should possess the following properties: (1) baryons as topological solitons, (2) a satisfactory description of low energy phenomenology in the meson sector and the baryon sector and (3) the correct nuclear force. A majority of recent works have concentrated on the search for possible effective Lagrangians phenomenologically, especially by nuclear physicists $[50,51,52,53,54,55,56]$.

The great theoretical fascination with the skyrme model is that it represents a 
dynamical system wherein one might hope to comprehend all low-energy hadronic phenomenology directly in terms of a nonlinear $\sigma$ model effective Lagrangian. Our goal is to be able to derive such an effective Lagrangian with few if any adjustable parameters.

Theoretical derivation of the effective Lagrangian has been hindered by the major problem of solving QCD at low energy and the lack of a systematic method for the effective action expansion. While solving QCD remains an outstanding problem there has been tremendous progress in finding a simple and systematic method for the effective action expansion. The availability of such a method would offer an opportunity to obtain effective Lagrangians by exploring various scenarios from QCD.

The spontaneously broken chiral symmetry scenario of quantum chromodynamics (QCD) together with the Veltman theorem implies that the low energy effective Lagrangian of QCD must be of the nonlinear $\sigma$ model type[49]. The effective Lagrangians resulting froin integrating the quark fields from the gauged or nongauged chiral quark model Lagrangian in a Skyrme-type scenario may be completely equivalent to $\mathrm{QCD}$ in the large $N_{\mathrm{c}}$ limit at low energy and therefore may serve as realistic models for low energy hadron dynamics.

For this purpose, I have presented in detail the recent development of the effective action expansion, in particular the evaluation of the fermion determinant of these models $[6,8,48]$. The implications on the low energy phenomenology associated with these effective Lagrangians have been discussed.

I plan to use Mathematica to generate the six and eight-derivative terms for the effective Lagrangian to investigate the effect on both the meson and the baryon dynamics, especially on whether these term can help to stabilize the skyrmion solution.

In a recent paper in Physics Letters, Novozhilov[57] calculated the effective QCD chiral Lagrangian for pions and vector mesons with the chiral symmetry group $G$ and the hidden local symmetry group $G_{h}=S U(2)_{h L} \times S U(2)_{h R}$ where the $\rho$ meson is the composite gauge field of the hidden local symmetry group[58]. The amazing result of his calculation is that he can determine the $\rho$ meson parameter completely, 


$$
g^{2}=\frac{12 \pi^{2}}{N_{c}}=4 \pi^{2}, m_{\rho}=g^{2} F_{\pi}^{2} \text { and } g_{\rho \gamma}=2 F_{\pi}^{2} g_{\rho \pi \pi},
$$

which are identical to what I have found from the effective Lagrangian of the chiral quark model albeit the $\rho$ meson is dynamically generated from the pion form factor rather than built in as the gauge meson of the hidden symmetry. It will be very interesting to find out how this two model are related and it may shed some light on the origin of the hidden symmetry.

\section{A.2.4 Berry's phases and the Wess and Zumino terms}

A very important progress in particle physics and quantum field theory in the recent years is the understanding of why some symmetries in classical physics may disappear when symmetry violating quantum processes are taken into account. The anomalous symmetry breaking term in the effective Lagrangian is known as the Wess-Zumino term[59]. The existence of the the Wess-Zumino terms or the anomaly depends on the highly nontrivial topology of the mapping between the internal symmetry space and the configuration space. It is of no surprise that the naive application of the derivative expansions, which describe the local variations, would not be able to deduce the Wess-Zumino term which own its existence to the nontrivial global properties.

Berry's elegant work[60] on the modification of the quantum adiabatic theorem provides a general framework to understand the non-integrable phase in dynamical system in which the Wess-Zumino term is a special case in quantum field theories[61]. Berry discovered that when a system traverse a closed path in an external parameters space, the wave function acquires an additional nonintegrable geometrical phase factor not included in the more familiar dynamical phase factor. This phase factor is amazingly universal [62]. Since quantum mechanics can be considered as a zero dimension field theory, adiabatic expansion is the derivative expansion, as the Berry's phase corresponds to the Wess-Zumino term, a quantum mechanics model in which Berry's phase is realized would provide a simple laboratory to explore our ideas of derivative expansion. Conversely our techniques in derivative expansion and topological consideration can be applied to give a simple understanding and 
derivation of the Berry's phase.

I have found that the derivative expansion method which I developed in Ref. [6] is the only method suitable for this purpose, although not anticipated at that time. In order to by-pass some technical problems, this systematical method contains a little twist, namely to functionally differentiate the fermion determinant before the derivative expansion and to functionally integrate back. However it can now be seen that the final functional integration can be used as an important way to tie the derivative expansion to to topological structure of the mapping between the internal space and the configuration space. This can be illustrated by a very simple model of a spin- $\frac{1}{2}$ particle (fast degrees of freedom) coupled to a time-dependent external magnetic field (slow degrees of freedom). The effective action can be defined by integrating out the fast degrees of freedom (the spin- $\frac{1}{2}$ field). The effective action term corresponding to the Berry's phase can then be obtained by the derivative expansion method thus described. The similarity between the Berry's phase and the Wess-Zumino term becomes obvious. Therefore the techniques can equally be applied to the calculation of the Wess-Zumino terms. A paper to report this work is in preparation.

\section{A.2.5 Derivative expansions for strong fields Quantum Electrodynamics}

In the recent years, attention has been drawn to the strong coupling QED in both $3+1$ and $2+1$ dimensions in view of possible phase transition and critical behavior $[64,65,63]$. The strong-coupling phase of QED must be a nonperturbative phenomenon that depends on the presence of a rather strong and varying background field. Numerical method has been used to investigate QED on a lattice by Kogut et al[65].

It has been suggested[63] that it may be a reasonable approximation to use Schwinger's proper-time formalism to study the fermion propagator in the presence of a background field. However it is shown that the analytical solution given by constant-field case is not rich enough to display the critical behavior. It is neccessary to incorperate the variation of the field into the calculation. For that purpose, I 
have been devising a method to integrate my derivative expansion technique with the Schwinger's proper timie formalism so that the leading term of the expansion corresponds to schwinger's analytic solution and the correction to the constantfield approximation can be calculated perturbatively in the order of the number of derivatives of the field.

Although the method I have is well-define, it would not be realistic to perform such calculation by hand. With slight modification of my new tensor program, It would made it possible to do this calculation on the computer. 


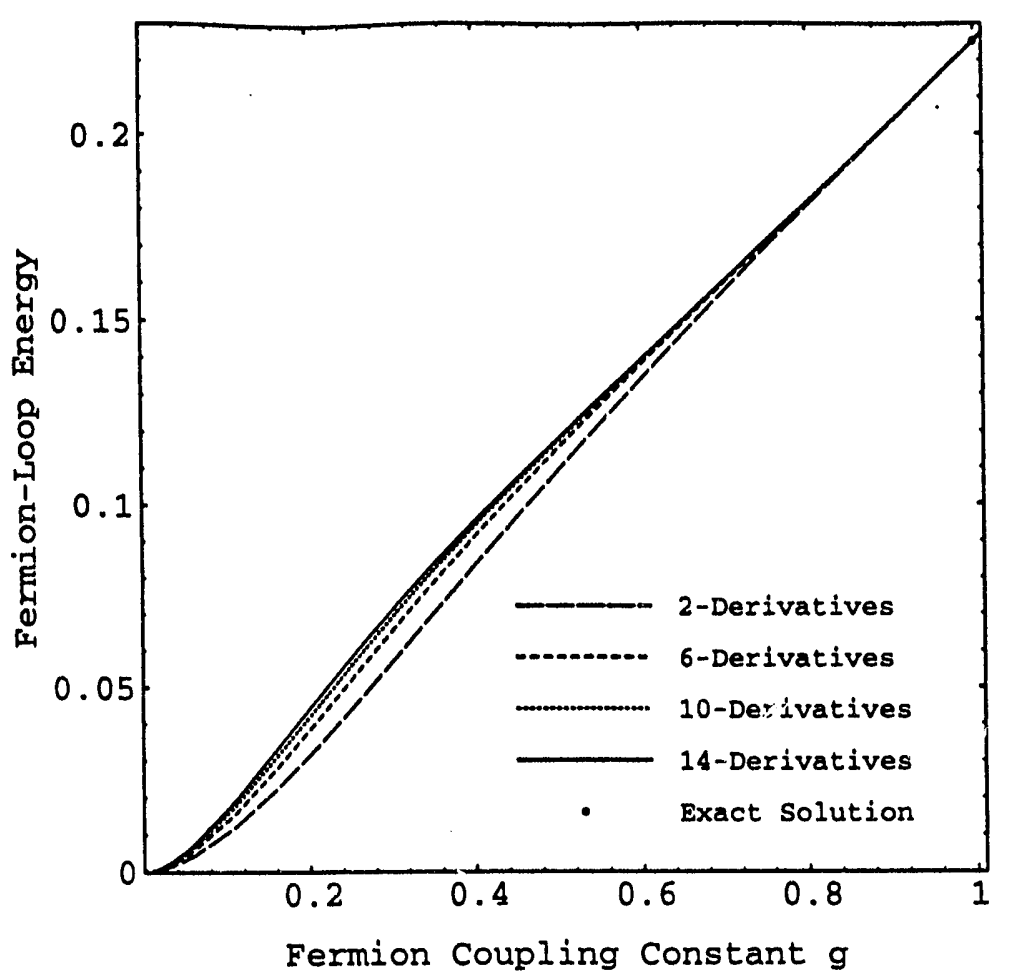

Fermion-Loop Quantum Correction to the phi-4 Soliton Energy

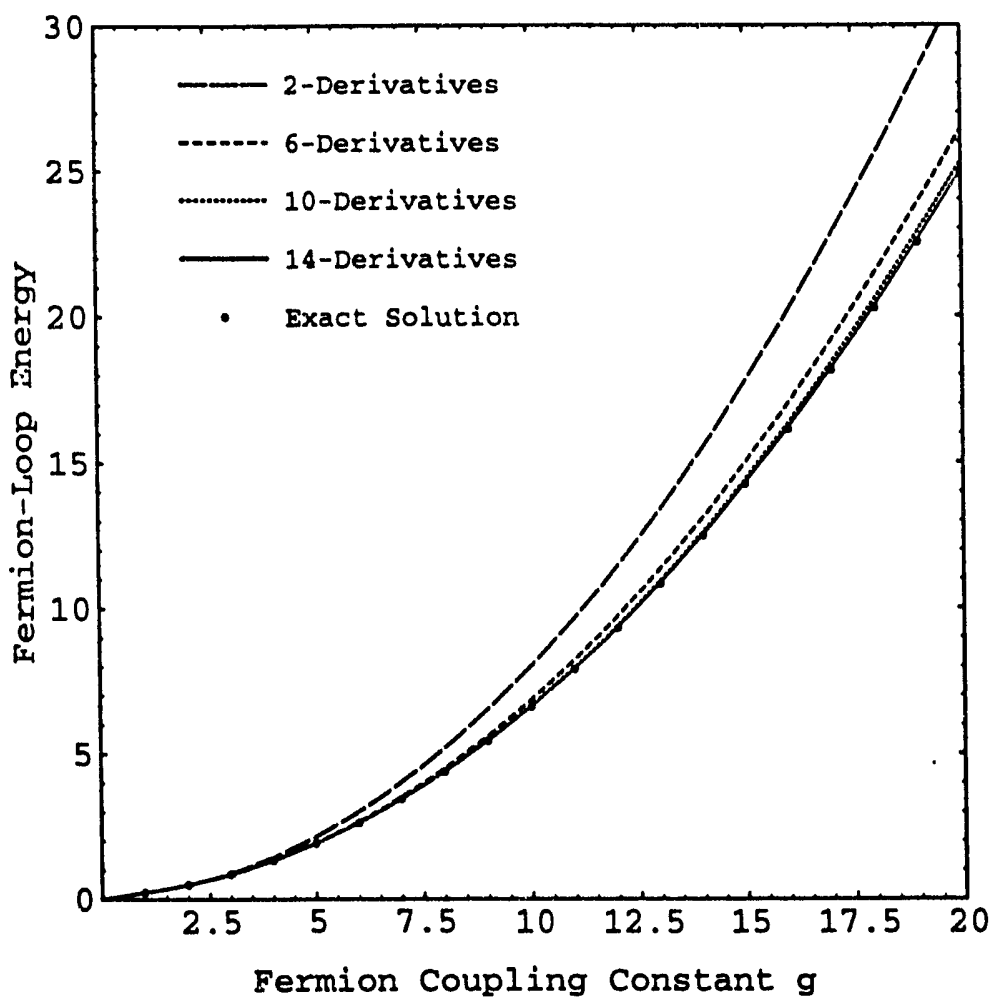

Fermion-Loop Quantum Correction to the phi-4 Soliton Energy 


\section{References}

[1] L.-H. Chan, Phys. Rev. D15, 2478 (1977).

[2] L.-H. Chan, Phys. Rev. Lett. 51,253 (1983).

[3] L.-H. Chan, Phys. Rev. D31, 204 (1985).

[4] L.-H. Chan, Phys. Lett. 71B , 422 (1977).

[5] L.-H. Chan, Phys. Rev. Lett. 55,21 (1985).

[6] L.-H. Chan, Phys. Rev. Lett. 54,1222 (1985); 56 , 404 (1985).

[7] L.-H. Chan, Phys. Rev. Lett. 57,1199 (1986).

[8] L.-H. Chan, Chiral Soliton Chapter 2, edited by K. F. Liu world Scientific, Singapore, 1987.

[9] L.-H. Chan, Phys. Rev. D36, 3755 (1986).

[10] L.-H. Chan, to be published in Phys. Rev. (1988).

[11] Stephen Wolfram, Mathematica - A System of Doing Mathematics by Computer, Addison Wesley (1988)

[12] Roman Maeder,Programming in Mathematica,Addison Wesley (1990)

[13] L.-H. Chan, Phys. Lett. 71B , 422 (1977).

[14] C. M. Fraser, Z. Phys. C28, 101 (1985).

[15] I. J. R. Aitchison and C. M. Fraser, Phys. Lett. 146B , 63 (1984); Phys. Rev. D 31, 2605 (1985).

[16] O. Cheyette, Phys. Rev. Lett. 55,2394 (1985).

[17] M. K. Gaillard, Nucl. P.iys. B268 , 669 (1986); (1986).

[18] M. Li. R. Perry and L. Wilets, Phys. Rev. D36, 596 (1987) .

[19] R. Perry and M. Li, Mod. Phys. Lett. A2, 353(1987).

[20] R. J. Perry, Phys. Lett. B182,269 (1986); University of Washington Preprint 40048-38-N6 (1986).

[21] D. A. Wasson and S. E. Koonin, Phys. Rev. D43, 3400 (1987) .

[22] O. Cheyette, M. K. Gaillard, Phys. Lett. B197, 205 (1987).

[23] M. K. Gaillard, Carese Summer School 1987:189.

[24] P. Binetruy, S. Dawson, M. K. Gaillard and I. Hinchliffe, Phys. Rev. D37, 2633 (1988).

[25] P. Binetruy and M. K. Gaillard, Nucl. Phys. B312, 341 (1989).

[26] P. Binetruy and M. K. Gaillard, Phys. Lett. B22 D, 68 (1989).

[27] M. K. Gaillard and V. Jain, Phys. Rev. D39, 37,i5 (1989).

[28] V. Jain, Phy. Rev. D40, 1213 (1989).

[29] R. Ball and H. Osborn, Nucl. Phys. B263, 245 । 1986).

[30] M. Li. R. Perry and L. Wilets, Phys. Rev. D36, ís (1987) .

[31] R. Perry and M. Li, Mod. Phys. Lett. A2, 353(1987). 
[32] R. J. Perry, Phys. Lett. B182,269 (1986); University of Washington Preprint 40048-38-N6 (1986).

[33] F. Cooper, G. S. Guralnik and S. H. Kadan, Phys. Rev. D14, 1607 (1976).

[34] C. M. Bender, F. Ccoper and G. S. Guralnik, Ann. Phys. (N. Y.) 109, 165 (1977).

[35] Y. Fujimoto, L. O'Raifeartaigh and G. Parrvicini, Nucl. Phys. B212, 268 (1983).

[36] J. M. Cornwall, R. Jackiw, and E. Tomboulis, Phys. Rev. D10, 2428 (1974).

[37] T. Barnes and G. I. Ghandour, Phys. Rev. D22, 924 (1980) .

[38] A. Okopińska, Phys. Rev. D35 1835, (1987).

[39] T. H. R. Skyrme, Proc. Roy. Soc. A260, 127 (1961) and A262, 237 (1961); G. Adkins, C. R. Nappi and E. Witten, Nucl. Phys. 228, 552 (1983); J. Goldstone and F. Wilczek. Phys. Rev. Lett. 47, 986 (1981); A. P. Balachandran, V. P. Nair and S. G. Rajeev, Phys. Rev. Lett. 49, 1124 (1982).

[40] E. Witten, Nucl. Phys. B223, 422 (1983); ibid 433 (1983);

[41] T. Appelquist and C. Bernard, Phys. Rev. D22, 200 (1980) and Phys. Rev. D23,425 (1981);

[42] J.M. Cornwall, Nucl. Phys. B157,392 (1979).

[43] R. Akhoury and Y. P. Yao, Phys. Rev. D25, 3361 (1982)

[44] I. J. R. Aitchison and C. M. Fraser, Phys. Rev. D32, 2190 (1985)

[45] J. Honerkamp, Nuci. Phys. B36, 130 (1972); L. Tataru, Phys. Rev. Di2,335 (1975); D. I. Kazookov, V. N. Pervushin and S. V. Pushkin, Teor. Mat. Fiz., 31, 169 (1977) [Theor. Math. Phys. (USSR) 31, 389 (1977)].

[46] "Effective Action Expansion for the Nonlinear $\sigma$ Model - the Normal Coordinate Method", L.-H. Chan W. F. Kao and T. Matsuki, LSU preprint, Oct. 1988.

[47] C. P. Burgess and G. Kunstatter,Princeton Institute of Advanced Study preprint IASSNSHEP 87/34 (1987); G.A. Vilkovisky, in Quantum Theory Gravity, edited by S. Christensen, (Adam Hilger, Bristol, 1984); G. A. Vikovisky, Nucl. Phys. B234 (1984) 125; B. S. DeWitt, to appear in E. S. Fradkin's 60 $0^{\text {th }}$ Birthday Volume, (Adam Hilger Lt., Bristol, 1986).

[48] E. D'Hoker and E. Farhi, Nucl. Phys. B248, 59 (1984); B248, 77 (1984).

[49] M. Veltman, Nucl. Phys. B123, 89 (1977).

[50] A. Jackson, A. D. Jackson and V. Pasquier, Nucl. Phys. A 432, 567 (1985).

[51] U. B. Kaulfuss and U.-G. Meissner, Phys. Rev. C30, 2058 (1985); Phys. Rev. D31, 3024 (1985).

[52] V. Vento, Phys. Lett. 153B, 198 (1985).

[53] R. Vinh Mau, M. Lacombe, B. Loiseau, W. N. Cottingham and P. Lisboa, Phys. Lett. B150, 259 (1985).

[54] H. M. Sommermann, H. W. Wyld and C. J. Pethick, Phys. Rev. Lett. 55, 476 (1985).

[55] U.-G. Meissner and I. Zahed, Phys. Rev. Lett. 56, 1035 (1986).

[56] M. Lacombe, B. Loiseau, R. Vinh Mau and W. N. Cottingham, Phys. Rev. Lett. 57, 170 (1986). 
[57] V. Y. Novozhilov, Phys. Lett. B228, 240 (1989).

[58] M. Bando, T. Kugo, S. Uehara, K. Yamawaki and T. Yanagida, Phys. Rev. Lett. 54, 1215 (1985).

[59] J. Wess and B. Zumino, Phys. Lett. 37B, 95 (1971).

[60] M. V. Berry, Proc. R. Soc., A392, 45 (1984).

[61] P. Nelson and L. Alvarez-Gaumé, Comm. Math. Phys. 99, 103 (1985); H.somoda, phys. Lett. 156B, 220 (1985), Nucl. Phys. B266, 410 (1986); A. Niemi and G. Semenoff, Phys Rev. Lett. 55, 927 (1985);56,1019 (1986), Phys. Lett. B175, 439 (1986); A. Niemi, G. Semenoff and Y.-S. Wu, Nucl. Phys. B276, 173 (1986).

[62] C. A. Mead and D. G. Truhlar, J. Chem. Phys. 70, 2284 (1984); F. Wilczek and A. Zee, Phys. Rev. Lett 52, 211 (1984); J. Moody, A. Shapere, and F. Wilczek, Phy. Rev. Lett 56, 893 (1986); H. Kuratsuji and S. Iida, Phys. Rev. Lett 56, 1003 (1986); D. Arovas, J. R. Schrieffer, and F. Wilczek, Phys. Rev. Lett 53, 722 (1984)

[63] D. G. Caldi, A. Chodos, K. Everding, and D. A. Owen, Phys. Rev. D39, 1432 (1989)

[64] T. Appelquist and D. Nash,Phys. Rev. Lett 60, 2575(1988); Appeiquist, M. J.Bowick, D. Karabali, and L C. R. Wijewardhana, Phys. Rev. D33, 3704 (1986);Appelquist, M. J.Bowick, E. Cokler,and L C. R. Wijewardhana, Phys. Rev. Lett 55, 1715 (1985)

[65] E. Dagotto, J. Kogut and A. Kocić, Phys. Rev. Lett62, 1083 (1989); J. Kogut, E. Dagotto and A. Kocić, Phys. Rev.Lett 60, 772 (1988); J. Kogut, E. Dagotto and A. Kocić, Phys. Rev.Lett 61, 2416 (1988); 


\section{B QCD Dynamics on the Lattice (R. Haymaker)}

The group of collaborators working in lattice $Q C D[1]-[16]$ and related continuum problems now includes three faculty members: J. Wosiek, at the Jagellonian University in Cracow, and at LSU Dana Browne who is working primarily in condensed matter physics and myself. A postdoc working in lattice gauge theory, Ken Yee, joined our group on July 1. There are two graduate students at LSU: V. Singh and Y. Peng who are nearing completion of their degrees. V. Singh will be leaving after the present semester. She has a postdoc job at MatScience in Madras. I would like to start with a brief summary of recent work and future directions of our efforts and then expand in the following sections:

Lattice Simulations of Color Flux: We have had an ongoing program for some time to study the color flux surrounding static charges making use of the LSU FPS array processor[1]-[11]. This work was presented in Seillac('87)[2], Zakopane('89)[3], Capri('89)[4], Rice Univ('90)[5], Tallahassee('90)[7], Tsukuba('91)[8] and Paris('92)[9]. This phase, i.e. the $\mathrm{SU}(2)$ gauge theory is essentially complete. We have made the first test of the Michael sum rules[17] which we discuss here. The final work on flux profiles is condensed in the Paris proceedings[9] and a draft of the final paper[11] is circulating among authors.

Topological Mechanism for Quark Confinement: Confinement has long been suspected to be a consequence of the topological nature of the QCD Vacuum. In the mid-1970's 't Hooft and Mandelstam suggested that confinement could be thought of as a dual Meissner effect, where the quarks played the role of magnetic monopoles in a Type-II superconductor, and color magnetic monopoles acted like Cooper pairs. Confinemeni appears to be linked to the condensation of magnetic monopoles. We have been successful in seeing the dual Abrikosov vortex is considerable detail[16,9]. In $U(1)$ we were able to measure the curl of the magnetic monopole current and verify that the London relation was satisfied, and we used it to measure the London penetration depth. These results were reported at conferences in Vienna, Paris[9] and Amsterdam[10]. We further showed that the fluxoid was quantized. We are just now getting results for the $\mathrm{SU}(2)$ gauge fixed theory and again find a strong signal for the curl of the monopole current. From our results we infer that the U(1) case is an extreem type II dual superconductor in which the coherence length is much smaller than the penetration depth. In the SU(2) case we have tentitavely concluded that the coherence length is of the order of the London penetration depth. This will be reported in Amsterdam('92)[16] conference. A paper on $\mathrm{SU}(2)$ is in preparation. 
Finite Size Scaling: In our work so far we have used lattice volumes large enough to assure that the string formation takes place. However there is a wealth of data on smaller volumes where there are analytic results for comparisons. We would like to build on our flux calculations to see if some of the transitions seen in these regions correspond to changes in the flux distribution as a function of the lattice volume. We have succeeded in generalizing the Michael sum rules to the case of finite volume and temperature and have checked the results with flux calculations. Two papers are in preparation.

\section{B-1 Lattice Simulations of Color Flux (with J. Wosiek, V. Singh)}

\section{B-1.1 Background}

In this calculation we measure the field energy densities by correlating the small plaquette with the Wilson loop. Full details of the simulation are given in Ref. [6], [1]-[11] which also contains further references. Further details of the flux profiles will appear in a companion paper Ref. [7]. By fixing our attention on the middle time slice of the Wilson loop, the time-like segments form world lines that approximate a static $q \bar{q}$ pair. The 3 space-space plaquettes measure the magnetic component of the energy density and similarly the 3 space-time plaquettes measure the electric components.

Before defining the flux calculation in more detail, we point out that the Wilson loops themselves are used to extract the transfer matrix eigenvalues which give the static quark potential and are further used to extrapolate the flux measurements to infinite time extent of the Wilson loops. Specifically we determine the eigenvalues of the transfer matrix by fitting the Wilson loops to the exponentials as described in Ref.[6].

$$
<W(R, T)>=\sum_{i} A_{i} e^{-E_{i}(R) T} .
$$

$E_{0}(R)$ is of special interest since it contains the static quark potential:

$$
E_{0}(R)=-\frac{\alpha}{R}+\sigma R+\frac{c(\beta)}{a(\beta)} .
$$

The term independent of $R$ is the self energy of the two quarks which does not scale but diverges as $a \rightarrow 0$. Since Wilson loops can be calculated quite accurately, the static potential is a useful physical quantity to check scaling and thereby determine the lattice spacing $a(\beta)$. All our data is consistent with standard values $a(2.3)=0.171 \mathrm{fm}, a(2.4)=0.128 \mathrm{fm}$ and $a(2.5)=0.089 \mathrm{fm}$. 


\section{B-1.2 Flux Tube Profiles}

The lattice observable needed to measure the flux is the following[18].

$$
\begin{aligned}
f^{\mu \nu}(x) & =\frac{\beta}{a^{4}}\left(\frac{\left\langle W P_{x}^{\mu \nu}\right\rangle}{\langle W\rangle}-\langle P\rangle\right) \\
& \approx \frac{\beta}{a^{4}}\left(\frac{\left\langle W P_{x}^{\mu \nu}-W P_{x}^{\mu \nu}\right\rangle}{\langle W\rangle}\right),
\end{aligned}
$$

where $W$ is the Wilson loop, $P_{x}^{\mu \nu}$ the plaquette located at $x, \beta=\frac{4}{g^{2}}$ and $x_{R}$ is a distant reference point. In the classical continuum limit

$$
f^{\mu \nu} \stackrel{a \rightarrow 0}{\longrightarrow}-\frac{1}{2}\left\langle\left(F^{\mu \nu}\right)^{2}\right\rangle_{q \bar{q}-v a c}
$$

where the notation $\langle\cdots\rangle_{q \bar{q}-v a c}$ means the difference of the average values in the $q \bar{q}$ and vacuum state. From now on we shall be using field components in Minkowski space and hence

$$
f^{\mu \nu} \rightarrow \frac{1}{2}\left(-B_{1}^{2},-B_{2}^{2},-B_{3}^{2} ; E_{1}^{2}, E_{2}^{2}, E_{3}^{2}\right)
$$

Correspondence between various components and $f^{\mu \nu}$ is standard: space-space plaquettes are magnetic, space-time plaquettes are electric. The energy and action densities are respectively

$$
\begin{aligned}
& \epsilon=\frac{1}{2}\left(E^{2}+B^{2}\right), \\
& \gamma=\frac{1}{2}\left(E^{2}-B^{2}\right) .
\end{aligned}
$$

Since the magnetic contribution turns out to be negative, there is a strong cancellation between the two terms in the energy, whereas they are enhanced in the action. Figure 1 gives the flux profiles. The cancellation which suppresses the energy density in the flux tube is evident. However notice that the self energy of the quarks is not similarly suppressed. This follows because the self energy is primarily electric. We fitted the energy and action density in the plane at the midpoint between $q$ and $\bar{q}$ using the function

$$
f\left(r_{\perp}\right)=a \exp \left(-\sqrt{b^{2}+\left(r_{\perp} / c\right)^{2}}\right) .
$$

The peak value and the width at half maximum were very well determined using a $\chi^{2}$ fit for each of 70 cases of different loop sizes and values of $\beta$. For the third parameter we chose the decay length of the tail of this function and found it less well determined but with a value typically close to the width at half maximum. The details of the analysis will be given in a forthcoming paper[11]. Here we 
just give the results of the extrapolation to infinite Wilson loop time extent in Figs. 2 and 3.

The basic issue is whether the peak value of the energy density stabilizes to a constant or goes to zero with quark separation. This is not easy to settle as can be seen in Fig. 2. Roughly speaking we know from the linearly rising potential that the string tension $\sim(\text { width })^{2} \times$ (peak value) should be constant. Both Figs. 2 and 3 show that we are marginally asymptotic in quark scparation, $R$. The two curves are $\sim 1 / R^{4}$ and $\sim 1 / R$. A Coulomb field would fall like the former but since the string tension is constant the asymptotic width would have to grow like $R^{2}$ which clearly it does not. Therefore we can rule out a Coulomb field as expected. Interestingly for small separations, the eyeball fit to $\sim 1 / R^{4}$ is quite good which may be due to a Coulomb like behavior at small distances. (The above argument that the width must grow like $R^{2}$ does not apply because there is no string for small $R$.) The dielectric model[28] predicts the peak density $\sim 1 / R$. This function (arbitrarily normalized) does not seem to fit the data very well. However such a behavior would imply the width $\sim \sqrt{R}$ which is certainly possible in our data. We can say that the peak energy density and width are consistent with a constant value for large quark separation but we can not rule out a slow variation. The issue can be tightened by making use of the Michael sum rules[17] as we mention in the next section.

Figure 4 illustrates a general feature of our data. The cluster of three points for each $R$ and $T$ correspond to the three quantities:

$$
\begin{aligned}
\epsilon & =\frac{1}{2}\left(E_{\|}^{2}+B_{\|}^{2}\right)+\frac{1}{2}\left(E_{\perp}^{2}+B_{\perp}^{2}\right), \\
\epsilon_{\|} & =\frac{1}{2}\left(E_{\|}^{2}+B_{\|}^{2}\right), \\
\epsilon(T \leftrightarrow R) & =\frac{1}{2}\left(E_{\|}^{2}+B_{\|}^{2}\right)-\frac{1}{2}\left(E_{\perp}^{2}+B_{\perp}^{2}\right) .
\end{aligned}
$$

If one turns the Wilson loop on its side, the $\|$ components are unchanged but the $\perp$ components of the electric and magnetic fields are reversed: $E_{\perp}^{2} \leftrightarrow-B_{\perp}^{2}$. Hence there is a sign change in the third expression. The central points of the cluster are the $\|$ components only. The clustering of the points implies that the $\perp$ components of the electric and magnetic contributions to energy density are approximately equal but of opposite sign and cancel. The width of the peak is even less sensitive to the transverse components giving essentially the same value for all three points. 


\section{B-1.3 Sum Rules}

A consistency check on the flux distributions can be obtained by using the Michael sum rules[17] for energy and action.

$$
\begin{aligned}
\frac{1}{2} \sum_{\vec{x}}\left(E(\vec{x})^{2}+B(\vec{x})^{2}\right) & =E_{0}(R) \\
\frac{1}{2} \sum_{\vec{x}}\left(E(\vec{x})^{2}-B(\vec{x})^{2}\right) & =-\beta \frac{\dot{a}}{a}\left[E_{0}(R)-\frac{c(\beta)}{a}\right]-\beta \frac{\dot{c}(\beta)}{a} .
\end{aligned}
$$

Here $E_{0}(R)$ is given by Eqn.(1), and $\left(\cdot \equiv \frac{d}{d \beta}\right)$. In ref.[6] we have shown that our data are essentially consistent with these sum rules. The one difficulty is the fact that the self energy, $c(\beta) / a(\beta)$, determined from the potential differs from the self energy determined from the the action sum rule. This may be due to an ambiguity in the definition of self energy or possibly due to our classical expressions for energy and action which ignores quantum corrections. By taking a derivative of these expressions with respect to the quark separation, $R$, this difficulty is avoided. This gives the relation

$$
\sigma_{A}=-\beta \frac{\dot{a}}{a} \sigma ; \quad \sigma_{A} \equiv \frac{1}{2} \sum_{\vec{x}_{\perp}}\left(E(\vec{x})^{2}-B(\vec{x})^{2}\right) ; \quad \sigma \equiv \frac{1}{2} \sum_{\vec{x}_{\perp}}\left(E(\vec{x})^{2}+B(\vec{x})^{2}\right) .
$$

The sums are now over the plane midway between the $q \bar{q}$ pair.

Using the sum rules we find that the $\beta$ function $(-\beta \dot{a} / a) \approx 10 . \pm 2$. compared to the current estimates $7 . \pm 1$.. The asymptotic value is $-51 / 121+3 \pi^{2} \beta / 11=$ $6.0(\beta=2.4)$. There is ample evidence from other measurements that although scaling works well, asymptotic scaling is violated[20] and hence we do not expect to get the asymptotic value.

An alternative approach is to assume the sum rules are correct and use them to infer information about the energy density from the action density which is far easier to measure since relative errors are down by an order of magnitude. As is clear from the sum rule, the action does not scale yet the variation over these values of $\beta$ is very small. An examination of Fig. 2(a) shows that the action for each $\beta$ seems to stabilize to a constant for increasing distance for the peak density and for the width. This is quite striking for $\beta=2.3$ and 2.4 . For $\beta=2.5 R$ appears to be too small to draw a conclusion. These data do not suggest that the peak value is tending to zero at all. We would like to use the sum rules to predict the behavior of the energy density. A constant peak energy density follows only if the widths of the energy and action peaks have the same behavior. Figure 3 shows that in fact they do. From this and using the sum rules we conclude that the energy density stabilizes to a constant value also. This conclusion is an argument against the dielectric model[28]. However we have little to say about logarithmic behavior of the flux tube width as predicted by Lüscher[19]. For more details see Ref.[7]. 


\section{B-2 Topological Mechanism for Quark Confinement (with V. Singh and D. Browne)}

\section{B-2.1 Dual Superconductor Model of Confinement}

We now turn to the mechanism for flux tube formation and present direct evidence that supercurrents of magnetic monopoles produce a dual Abrikosov vortex[21]. $U(1)$ lattice gauge theory in 4 dimensions has both a confined phase at large charge and a weak coupling deconfined phase corresponding to continuum electrodynamics with a Coulomb interaction between static charges. Therefore confinement or its absence can be studied using $U(1)$ lattice gauge theory as a prototype, before tackling the more complicated non-Abelian theories that actually describe quarks. Much evidence for the dual superconductor hypothesis has accumulated from studies[22,23,24] of lattice gauge theory. Polyakov[22] and Banks, Myerson and Kogut[23] showed that U(1) lattice gauge theory in the presence of a quark-antiquark pair could be approximately transformed into a model describing magnetic current loops (the monopoles) interacting with the electric current generated by the $q \bar{q}$ pair. DeGrand and Toussaint[24] demonstrated via a numerical simulation that the vacuum of $U(1)$ lattice gauge theory was populated by monopole currents, copious in the confined phase and rare in the deconfined phase. This behavior has also been seen in non-Abelian models after gauge fixing[25]. Many studies of non-Abelian models using Dirac monopoles[25,26] or other topological excitations[27] support the dual superconductor mechanism, although other studies[29] dissent.

So far, studies of confinement have examined "bulk" properties such as the monopole density[24,25], and the behavior of the static quark potential[26]. In a recent paper[16] we presented the first direct evidence that the flux tube is a dual Abrikosov vortex. We further show that there are exact $U(1)$ lattice gauge theory analogues of two key relations that lead to the Meissner effect in a superconductor; the London equation and the fluxoid quantization condition.

\section{B-2.2 Electric Field Profiles}

Our simulations were done on a Euclidean spacetime lattice of volume $9^{3} \times 10$. The static charges are represented by a Wilson loop as in the previous section. We take a $3 \times 3$ loop in the $z-t$ plane and measure the fields in the $x-y$ plane at the midpoint between the charges. Because of the geometrical symmetry of the measurements only the $z$-components of $\langle\overrightarrow{\mathcal{E}}\rangle$ and $\left\langle\vec{\nabla} \times \vec{J}_{M}\right\rangle$ are nonzero. If the Wilson loop is removed, even the $z$-components average to zero, so the response is clearly induced by the presence of the static charges. Only the imaginary part of the Wilson loop contributes to the averages of these two quantities. 
The plaquette measures flux passing through a unit square on the lattice ${ }^{1}$

$$
\exp \left[i e a^{2} F_{\mu \nu}(\vec{r})\right]=\exp \left[i \theta_{\mu \nu}(\vec{r})\right] \equiv U_{\mu}(\vec{r}) U_{\nu}(\vec{r}+\mu) U_{\mu}^{\dagger}(\vec{r}+\nu) U_{\nu}^{\dagger}(\vec{r}) .
$$

The electric flux in lattice variables is

$$
\mathcal{E}_{\mu}(\vec{r})=\operatorname{Im} \exp \left[i \theta_{\mu 4}(\vec{r})\right] .
$$

Figure 5(a) shows the electric flux distribution for $\beta=1.1$ where the vacuum is in the deconfined phase. The broad flux distribution seen is identical to the dipole field produced by placing two classical charges at the quark positions, except that the classical value of the flux on the $q \bar{q}$ axis is a factor of two smaller. We measure the total electric flux from one quark to the other, including not only the flux through the plane between the charges $(0.8504 \pm 0.0045)$ but also the flux $(0.0951 \pm 0.0028)$ that flows through the lattice boundary because of the periodic boundary conditions. This yields a total flux of $(0.9453 \pm 0.0053)$, ciose to the theoretical value $\Phi_{e}=e / \sqrt{\hbar c}=1 / \sqrt{\beta}=0.9534$.

Figure 5(b) and 6(a) shows the electric flux in the confined phase $(\beta=0.95)$. In this case the flux is confined almost entirely within one lattice spacing of the axis and essentially no flux passes the long way around through the lattice boundary. The net flux is again equal to $1 / \sqrt{\beta}$ within statistical error. This behavior is exactly what one would expect from the superconducting analogy, where the flux has been "squeezed" into a narrow tube.

\section{B-2.3 Magnetic Monopole Supercurrents}

The monopole currents are found by a prescription devised by DeGrand and Toussaint[24], which employs a lattice version of Gauss' Law to locate the Dirac string attached to the monopole. The net flux into each plaquette face is given by $\left(\theta_{\mu \nu}(\vec{r}) \bmod 2 \pi\right)$. If the sum of the fluxes into the faces of a 3-volume at fixed time is nonzero, a monopole is located in the box. A non-zero net flux can occur only if a multiple of $2 \pi$ arises from the mod operation. Therefore the net flux is

$$
\sum_{6 f a c e s} a^{2} F_{\mu \nu}=n(2 \pi \hbar c / e)=n e_{M}
$$

The net flux into the box at fixed time thus yields the monopole "charge" density, the time component of the monopole 4-current $J_{M}$. The spatial components are found similarly. The monopole currents form closed loops due to the conservation of magnetic charge. Finally the curl is calculated by the line integral of the current around a dual plaquette.

\footnotetext{
${ }^{1}$ The flux here means $E \times$ (area). We use the same term for $E^{2}$ or $B^{2}$ since it has become an accepted usage.
} 
We show in Fig. 6(a) $\langle\overrightarrow{\mathcal{E}}\rangle$ and in Fig. $6(\mathrm{~b})-\left\langle\vec{\nabla} \times \vec{J}_{M}\right\rangle$ in the confined phase as a function of the distance from the $q \bar{q}$ axis. The data show that the spatial variation of the flux and the curl of the current are very similar, except for the point on the axis which will be discussed below.

\section{B-2.4 London Equations, Fluxoid Quantization and the Abrikosov Vortex}

In order to interpret this result we would like to review the ordinary London theory. In the next section we interchange electric and magnetic quantities to get the dual results.

A concise statement of the London theory is contained in the relation ${ }^{2}$

$$
\vec{A}+\frac{\lambda^{2}}{c} \vec{J}=0 ; \quad(\vec{\nabla} \cdot \vec{A}=0) .
$$

If the charge density is zero, then in this gauge the electric field is given by $-\dot{A} / c$ and therefore $\vec{E}=\lambda^{2} \vec{J} / c$. This describes a perfect conductor and is just Newton's law for free carriers, $e \vec{E}=m \dot{\vec{v}}$. By taking the curl of Eqn.(14) we obtain the condition for a perfect diamagnet.

$$
\vec{\nabla} \times \vec{J}=-\frac{c}{\lambda^{2}} \vec{B}
$$

This relation together with Ampere's law $\vec{\nabla} \times \vec{B}=\vec{J} / c$ gives $\nabla^{2} \vec{B}=\vec{B} / \lambda^{2}$ which implies that the magnetic field falls off in the interior of the superconductor with a skin depth $\lambda$.

Finally the fluxoid is given by the integral

$$
\int_{S}\left(\vec{B}+\frac{\lambda^{2}}{c} \vec{\nabla} \times \vec{J}\right) \cdot \hat{n} d a=\int_{C}\left(\vec{A}+\frac{\lambda^{2}}{c} \vec{J}\right) \cdot d \vec{l}=n \frac{\hbar c}{2 e}=n \Phi_{m}
$$

If the curve $C$ is in a simply connected region of a superconductor, then $n=0$. However if the curve encircles a hole in the material then $\mathrm{n}$ need not be zero but must be an integer. In an extreme type II Abrikosov vortex, a very small core is comprised of normal material. A single unit of magnetic flux of radius $\sim \lambda$ passes through the vortex. The fluxoid density $\vec{B}+\frac{\lambda^{2}}{c} \vec{\nabla} \times \vec{J}$ is zero everywhere except in the region of the normal material. In the limit in which the core is a delta function we obtain:

$$
\vec{B}+\frac{\lambda^{2}}{c} \vec{\nabla} \times \vec{J}=\Phi_{m} \delta^{2}\left(x_{\perp}\right) \hat{n}_{z} .
$$

Further if we use Ampere's law we can get an analytic expression for $B_{z}$.

$$
B_{z}\left(r_{\perp}\right)-\lambda^{2} \nabla^{2} B_{z}=\Phi_{m} \delta^{2}\left(x_{\perp}\right) ; \quad B_{z}=\frac{\Phi_{m}}{2 \pi \lambda^{2}} K_{0}\left(r_{\perp} / \lambda\right) .
$$

\footnotetext{
${ }^{2}$ We use Heaviside-Lorentz units to be consistent with lattice gauge theory.
} 


\section{B-2.5 Dual Superconductor}

We interpret our results using the following relations which are the dual of the corrsponding relations in the previous section:

$$
\vec{E}-\frac{\lambda^{2}}{c} \nabla \times \overrightarrow{J_{m}}=\Phi_{e} \delta^{2}\left(x_{\perp}\right) \hat{n}_{z} .
$$

where the unit of electric flux $\Phi_{e}=1 / \sqrt{\beta}$. Figure 5(c) shows the result of fitting our data to this relation. The London penetration depth, $\lambda$, is the only free parameter. We are able to determine a value of $\lambda$ which gives zero away from the axis and a delta function with the correct coefficient on axis. Further we can check that the electric flux profile is given by

$$
E_{z}=\frac{\Phi_{e}}{2 \pi \lambda^{2}} K_{0}(r / \lambda)
$$

This function has no free parameters. This curve is also shown in Fig. 6(a) showing excellent agreement. We find a value of $\lambda / a=1.19 \pm 0.02$, which is consistent with the range of penetration of the electric flux in Fig. 6(a) and the thickness of the current sheet in Fig. 6(b). We expect that, as in a superconductor, the transition to the deconfined phase will be signalled by a divergence of the London penetration depth. We have therefore measured $\lambda$ further from the deconfinement transition at $\beta=0.90$, and find a smaller penetration depth of $\lambda / a=0.78 \pm 0.05$. In the deconfined phase, $\beta=1.1$ we find an almost insignificant value of $\left\langle\vec{\nabla} \times \vec{J}_{M}\right\rangle$ and fitted values of $\lambda$ were larger than our lattice size.

In summary, one can find a value of the London penetration depth, $\lambda$, that satisfies Eqn.(19) off axis. One then finds that for the point on axis, the same value of $\lambda$ gives one quantum of electric flux as predicted by Eqn.(19). Finally the same value of $\lambda$ gives a good fit to the profile using Eqn.(20). Hence considerable detail of the dual Abrikosov is verified. It is perhaps surprising that a nonlinear, strongly interacting, model such as $U(1)$ lattice gauge theory could be described by such a simple model as the linear London equations but our results indicate that the operators $\langle\overrightarrow{\mathcal{E}}\rangle$ and $\left\langle\vec{\nabla} \times \vec{J}_{M}\right\rangle$ when measured in the presence of source of external flux like a Wilson loop, give an unambiguous indication of the confinement of electric flux by a monopole current distribution. The simulation yields a large signal even with modesnt amounts of computer time on a Sun workstation. Although the Meissner effect itself requires only that Eq. (20) hold off axis, our data also support the more restrictive fluxoid quantization relation on axis. This additional relation reflects the single-valued nature of the order parameter in a Ginzburg-Landau description of the monopole condensate. Because the monopoles appear pointlike in our simulations, lattice gauge theory looks like an extreme type-II superconductor.

We now also have results for SU(2). Figure 7 and 8 show the electric field and curl of the monopole current defined in the maximal abelian gauge[25]. It is 
very interesting that the curl of the magnetic monopole current has a behavior that can easily be interpreted as a coherence length defined in the GinzburgLandau theory as shown by the curve. Therefore we believe that both the London penetration depth and the coherence length can be measured for QCD. We believe this is a very interesting development and plan to scale up this calculation to investigate all aspects of this.

\section{B-3 Finite Size Scaling (with Y. Peng)}

In our work so far we have used lattice volumes large enough to assure that the string formation takes place. However there is a wealth of data on smaller volumes where there are analytic results for comparisons. A measure of the effect of the finite size of the lattice is to consider the dimensionless variable $z=L / \xi$, where $L$ is the length characterizing the lattice, and $\xi$ is the correlation length. 'Small' volumes are those for which $z<1$. There are analytic results for glueball mass ratios by Lüscher and Münster[32] available in this region using a perturbative method on a low energy effective Hamiltonian for SU(N) gauge theory on a torus. For larger values of $z$ these methods break down because one must take into account tunneling between different 'toron sectors' which great'y complicate the problem. Van Baal and Kohler[34,33] were able to solve this model in the intermediate volume region, i.e. where $1<z<5$ by introducing non-perturbative boundary conditions on the Rayleigh-Ritz trial functions. In these regions there is a remarkably good agreement between the lattice simulations and the continuum effective hamiltonian models. For larger volumes however there still are interesting phenonena taling place $[35,36]$. In particular monte carlo data show that the $E^{+}$glueball increase for $z$ in the range $5-10$ by a factor of $1.5[34]$. It is also believed that string formation occurs in this region of $z$.

We would like to build on our flux calculations to see if some of the transitions seen in these regions correspond to changes in the flux distribution as a function of the lattice volume. Our investigations are centered on the region of $5<z<20$ to see if it is accompanied by a clear signal in the flux distribution. Our results are organized around the Michael sum rules[17]. Two papers are in preparation.

\section{References}

[1] J. Wosiek and R. Haymaker, Phys. Rev. Rapid Commun. D 36, 3297 (1987).

[2] J. Wosiek, Proceedings of the Lat87 Conference in Seillac, Oct. 1887, Nucl. Phys. B (Proc. Suppl.) 4, 52 (1988). 
[3] R. W. Haymaker and J. Wosiek, Acta Physica Polonica B 21, 403 (1990).

[4] R. W. Haymaker, Y. Peng, V. Singh and J. Wosiek, Proceedings of the Lattice '89 Capri Conference Nucl.Phys. B (Proc. Suppl.) 17, 558 (1990).

[5] R. W. Haymaker, Y. Peng, V. Singh and J. Wosiek, Proceedings of the Annual Meeting of the Division of Particles and Fields of the APS, Houston 1990 World Scientific (1990) p. 789.

[6] R. W. Haymaker and J. Wosiek, Phys. Rev. D 43, 2676 (1991).

[7] R. W. Haymaker, V. Singh and J. Wosiek, Proceedings of The 1990 Tallahassee International Conference on Lattice Field Theory Nucl Phys. B (Proc. Suppl) 20, 207 (1991).

[8] T. Barczyk, R.W. Haymaker, V. Singh, E. Laermann and J. Wosiek, Proceedings of The 1991 Tsukuba International Conference on Lattice Field Theory Nucl Phys. B (Proc. Suppl) 26, 462 (1992).

[9] R. W. Haymaker, V. Singh, D. Browne and J. Wosiek, Proceedings of the 1992 Paris Conference on the QCD Vacuum, June 1-5, 1992, to be published by World Scientific.

[10] V. Singh, D. Browne and R.W. Haymaker, Proceedings of The 1992 Amsterdam International Conference on Lattice Field Theory to be published in Nucl Phys. B (Proc. Suppl)

[11] R. W. Haymaker, V. Singh and J. Wosiek, Paper in preparation.

[12] R. W. Haymaker and J. Wosiek, Phys. Rev. D37, 969,(1988).

[13] R. W. Haymaker and J. Wosiek, Nucl. Phys. B (Proc. Suppl.) 4, 571, 1988).

[14] R. W. Haymaker and Y. Peng, Phys. Rev. D 41, 1269, (1990).

[15] R. Haymaker and Y. Peng, Proceedings of the Annual Meeting of the Division of Particles and Fields of the APS, Houston 1990 World Scientific (1990) p. 791.

[16] V. Singh, R. W. Haymaker and D. Browne, LSU Preprint LSUHEP no 1-92, May 1992.

[17] C. Michael, Nucl. Phys. B 280 [FS18] 13, (1987).

[18] M. Fukugita and T. Niuya, Phys. Lett. B 132, 374 (1983); R. Sommer, Nucl. Phys. B 291, 673 (1987); B 306, 180 (1988); I. H. Jorysz and C. Michael, Nucl. Phys B 302, 448 (1987); C. Michael and M. Teper, Nucl. Phys. B 305, 453 (1988); N. A. Campbell, A. Huntley and C. Michael, Nucl. Phys. B 206, 51 (1988); S. Perantonis, A. Huntley and C. Michael, Nucl. Phys. B326, 544 (1989); W. Feilmair and H. Markum, Nucl. Phys. B 370, 299 (1992); W. Burger, M. Faber, W. Feilmair, H. Markum and M Muller, Nucl. Phys. B20 (Proc. Suppl.), 203, (1991);

[19] M. Lüscher, Nucl. Phys.B 180 [FS2], 317 (1981); M. Lüscher, G. Münster and P. Weisz, Nucl. Phys. B180[FS2], 1 (1981).

[20] C. Michael, Phys. Lett. B283, 103, (1992).

[21] H. B. Nielsen and P. Olesen, Nucl. Phys. B61, 45 (1973); J. Kogut and L. Susskind, Phys. Rev. D 9, 3501 (1974); S. Mandelstam, Phys. Rep. 23C, 245 (1976); G. 't Hooft, in High Energy Physics, ed. A. Zichichi (Editrice Compositori, Bologna, 1976), Nucl. Phys. B100[FS3], 455 (1981). 
[22] A. M. Polyakov, Phys. Lett. 50B, 82 (1975).

[23] T. Banks, R. J. Myerson, and J. Kogut, Nucl. Phys. B129, 493 (1977).

[24] T. A. DeGrand and D. Toussaint, Phys. Rev. D 22, 2478 (1980).

[25] A. S. Kronfeld, M. L. Laursen, G. Schierholz, and U.-J. Wiese, Phys. Lett. 198B, 516 (1987); Nucl. Phys. B293, 461 (1987); F. Brandstaeter, G. Schierholz, and U.-J. Wiese, Phys. Lett. 272B, 319 (1991); M. Teper, Phys. Lett. 171B, 86 (1986); R. J. Wensley and J. D. Stack, Phys. Rev. Lett. 63, 1764 (1989).

[26] T. Suzuki and I. Yotsuyanagi, Phys. Rev. D 42, 4257 (1990).

[27] J. Smit and A. J. van der Sijs, Nucl. Phys. B355, 603 (1991); T. L. Ivanenko, A. V. Pochinsky, and M. I. Polikarpov, Phys. Lett. 252B, 631 (1990).

[28] S. L. Adler, Nucl. Phys. B 217, 381 (1983).

[29] J. Greensite and J. Winchester, Phys. Rev. D 40, 4167 (1989); A. Di Giacomo, M. Maggiore, and S. Olejnik, Nucl. Phys. B347, 441 (1990).

[30] G. A. 'tHooft, Nucl. Phys. B138, 1, (1978); ibid. B153, 141, (1979).

[31] M. Lüscher, Phys. Lett. 118B, 391, (1982); Nucl. Phys. B219, 233, (1983).

[32] M. Lüscher and G. Münster, Nucl. Phys. B232, 445, (1984).

[33] P. Van Baal and J. Koller, Ann. Phys. (N.Y.) 174, 299, (1987); Nucl. Phys. B302, 1, (1988).

[34] P. Van Baal, Nucl. Phys. B9,(Proc. Suppl),227, (1989).

[35] B. A. Berg and A. H. Billoire, Phys. Rev. D40, 550, (1989).

[36] C. Vohwinkel and B. A. Berg, Phys. Rev. D40, 584, (1989). 

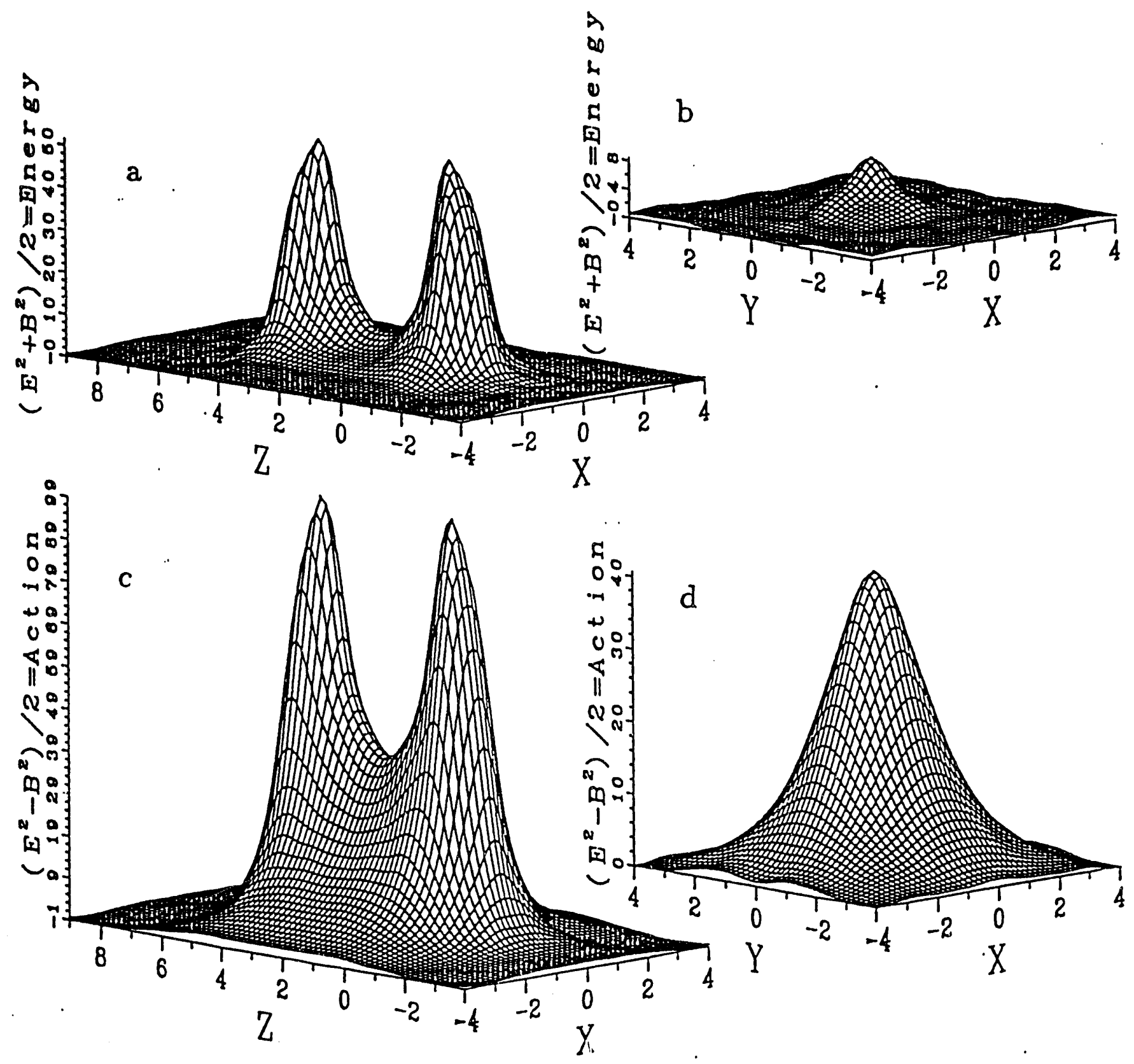

Figure 1: Energy and action profiles: (a) energy density in the plane containing $q \bar{q}$; (b) energy density in the plane midway between $q$ and $\bar{q}$; (c) and (d) similarly for action density. 

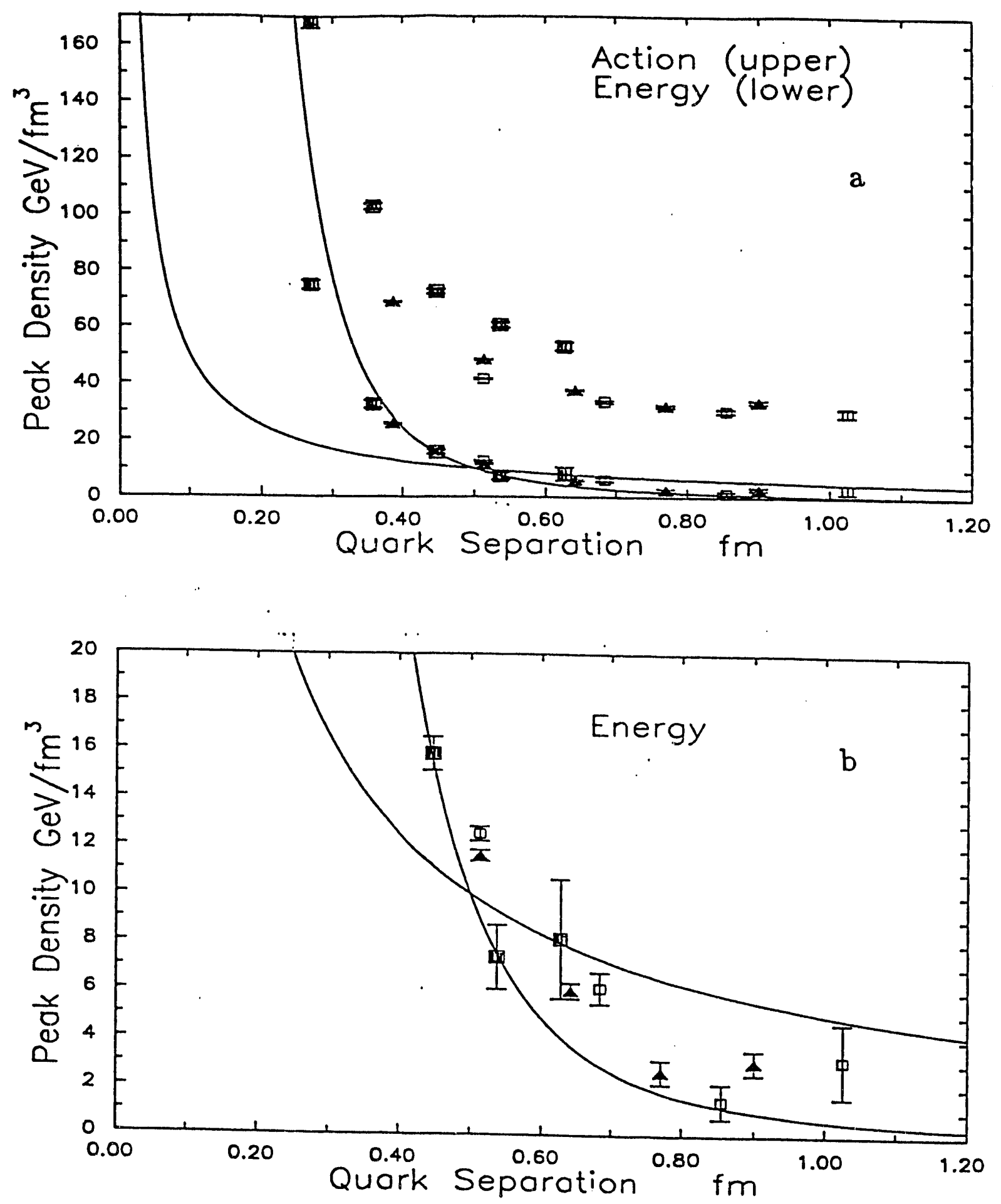

Figure 2: (a) Peak value of energy and action density; solid squares: $\beta=2.5$; triangles: $\beta=2.4$; open squares: $\beta=2.0$. The two curves are $1 / R$ and $1 / R^{4}$ arbitrarily normalized; (b) blowup of (a). 


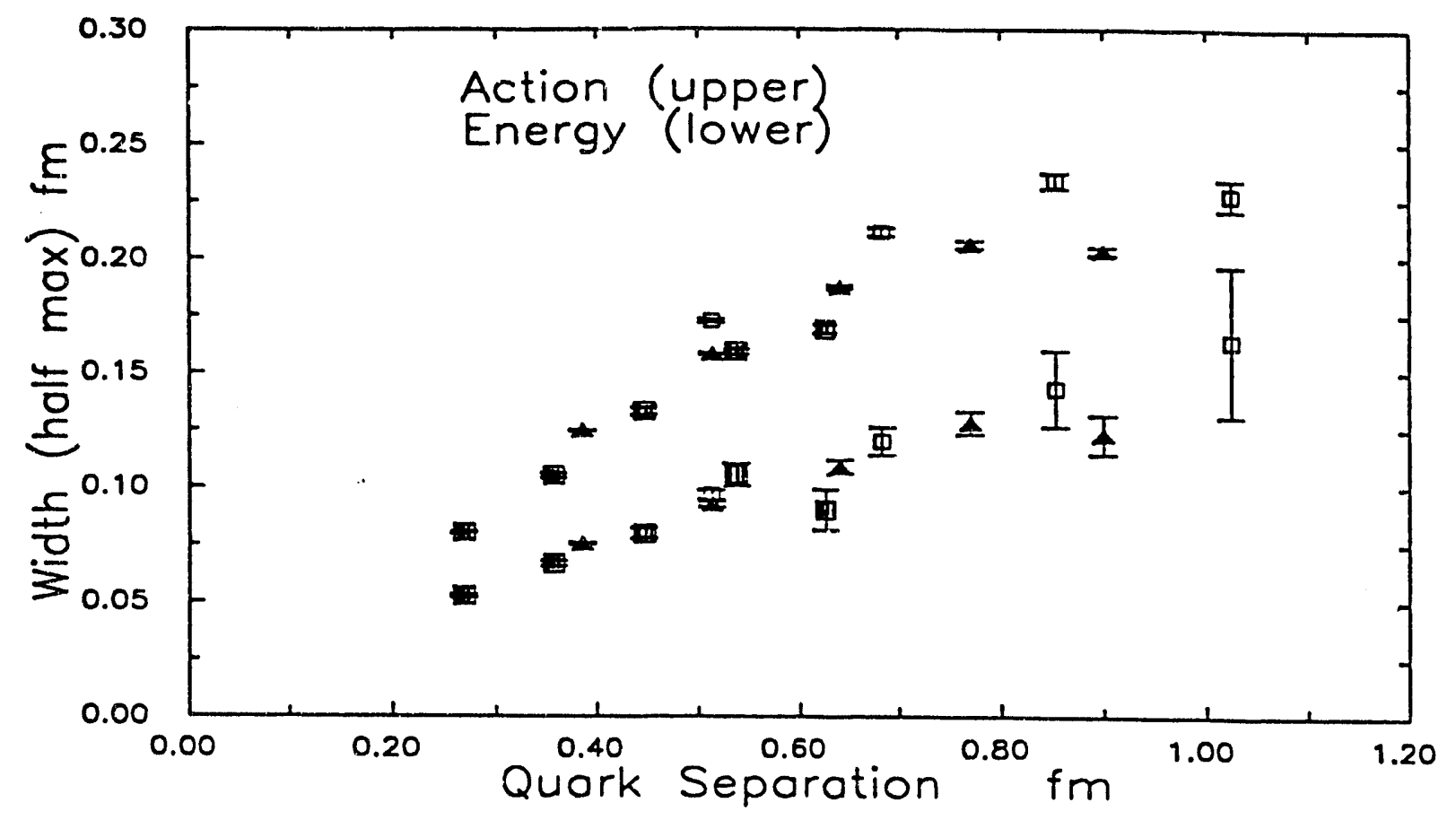

Figure 3: Width at half maximum for energy and action density. 


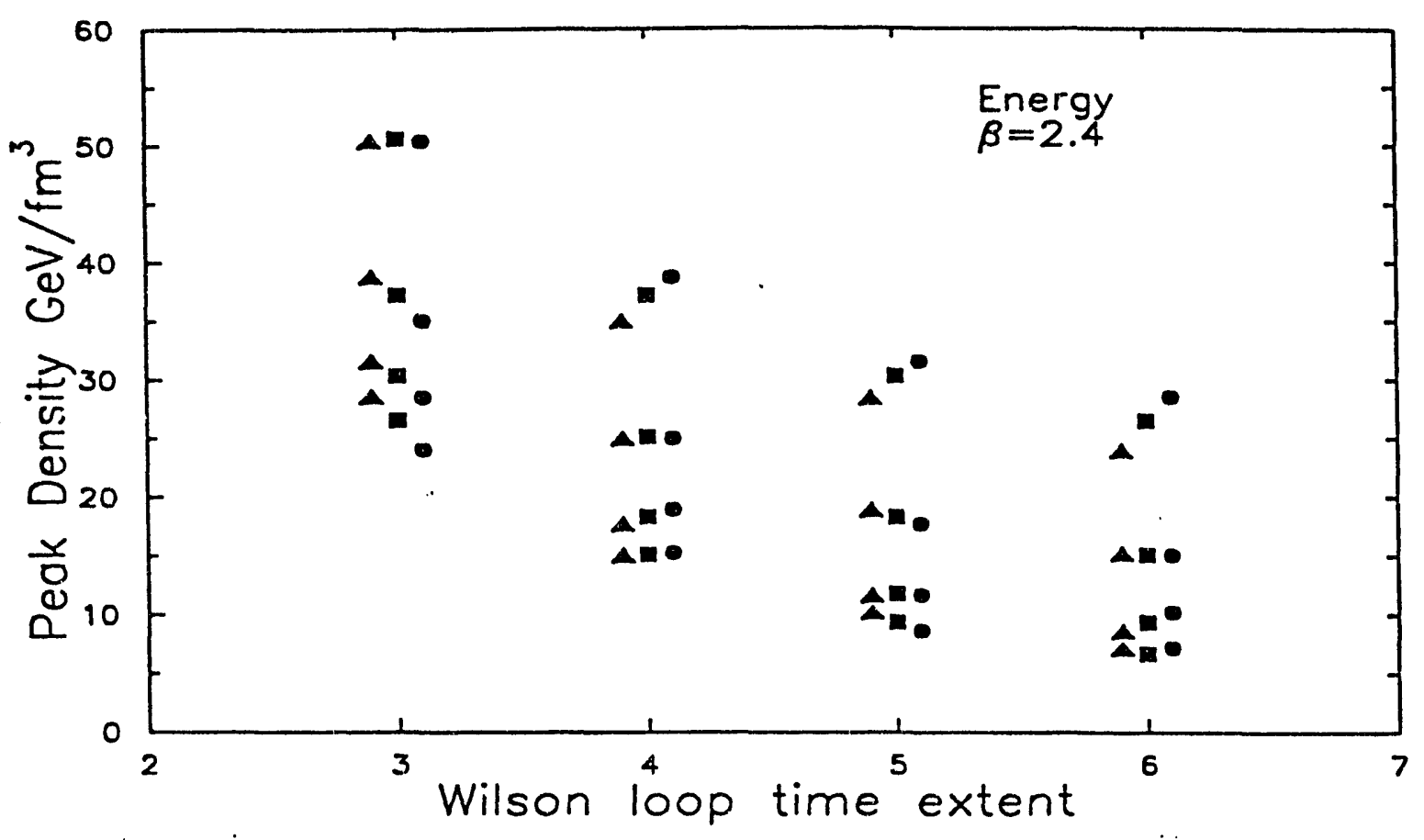

Figure 4: Peal density for $R \times T$ Wilson loop sizes $R=3-6, T=3-6$. For fixed $T$ the points decrease monotonically with $R$. Triangles: energy density for $R \times T$ loop; circles: energy density for $T \times R$ loop; squares: \| components of $E^{2}$ and $B^{2}$ only. 

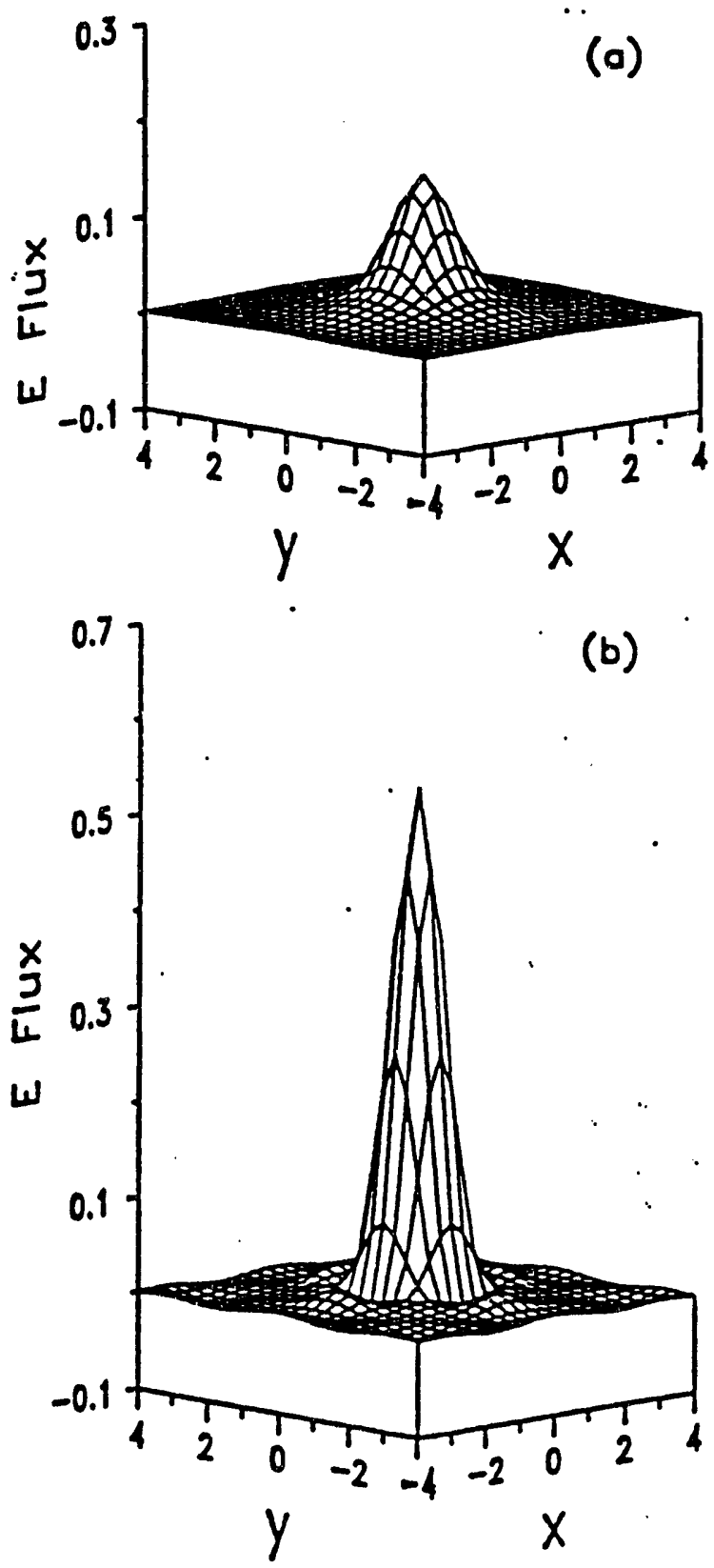

Figure 5: Surface plot of the electric flux through the $x y$ plane midway between the $q \bar{q}$ pair when the system is in (a) the deconfined phase $(\beta=1.1)$ and (b) the confined phase $(\beta=0.95)$. The line joining the pair is located at $(0,0)$. 


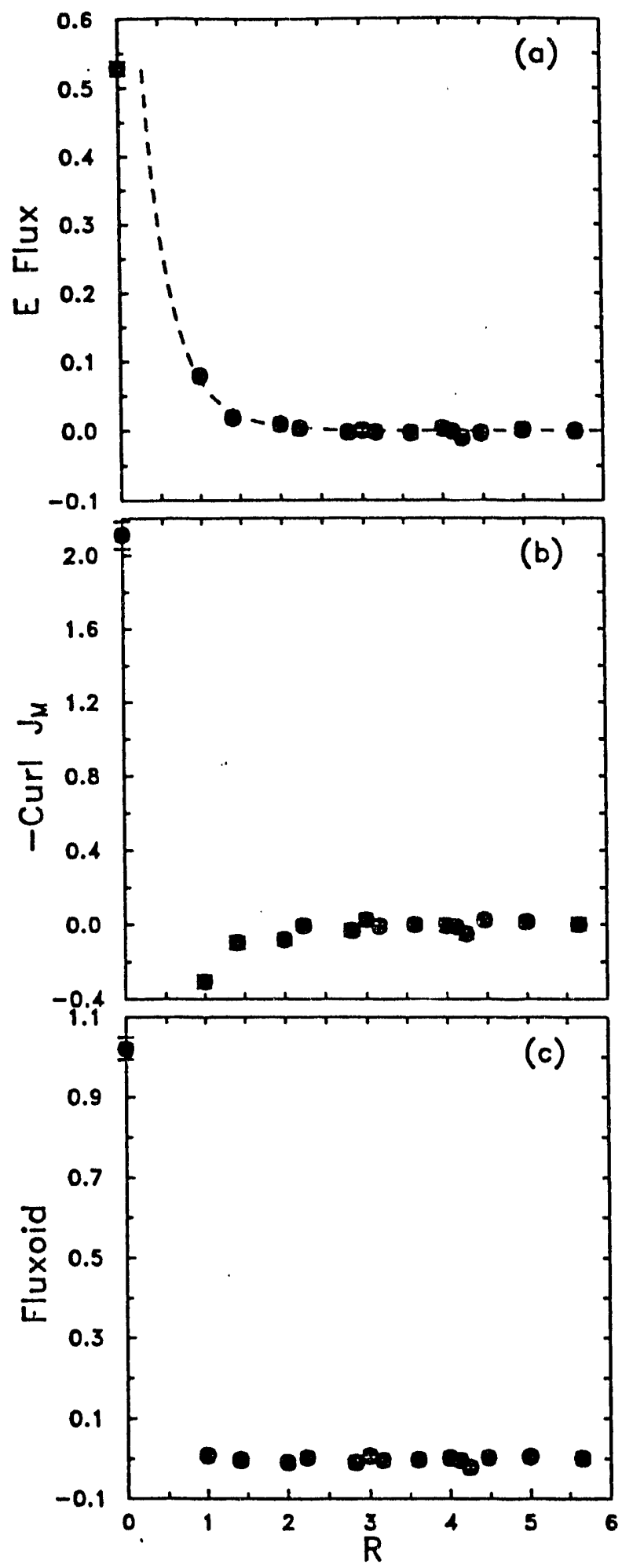

Figure 6: Behavior of (a) the electric flux, (b) the curl of the monopole current and (c) their sum, the fluxoid, in the confined phase $(\beta=0.95)$ as a function of the perpendicular distance $r_{\perp}$ from the $q \bar{q}$ axis. The solid line in (a) gives the flux for a pure Coulomb field. 


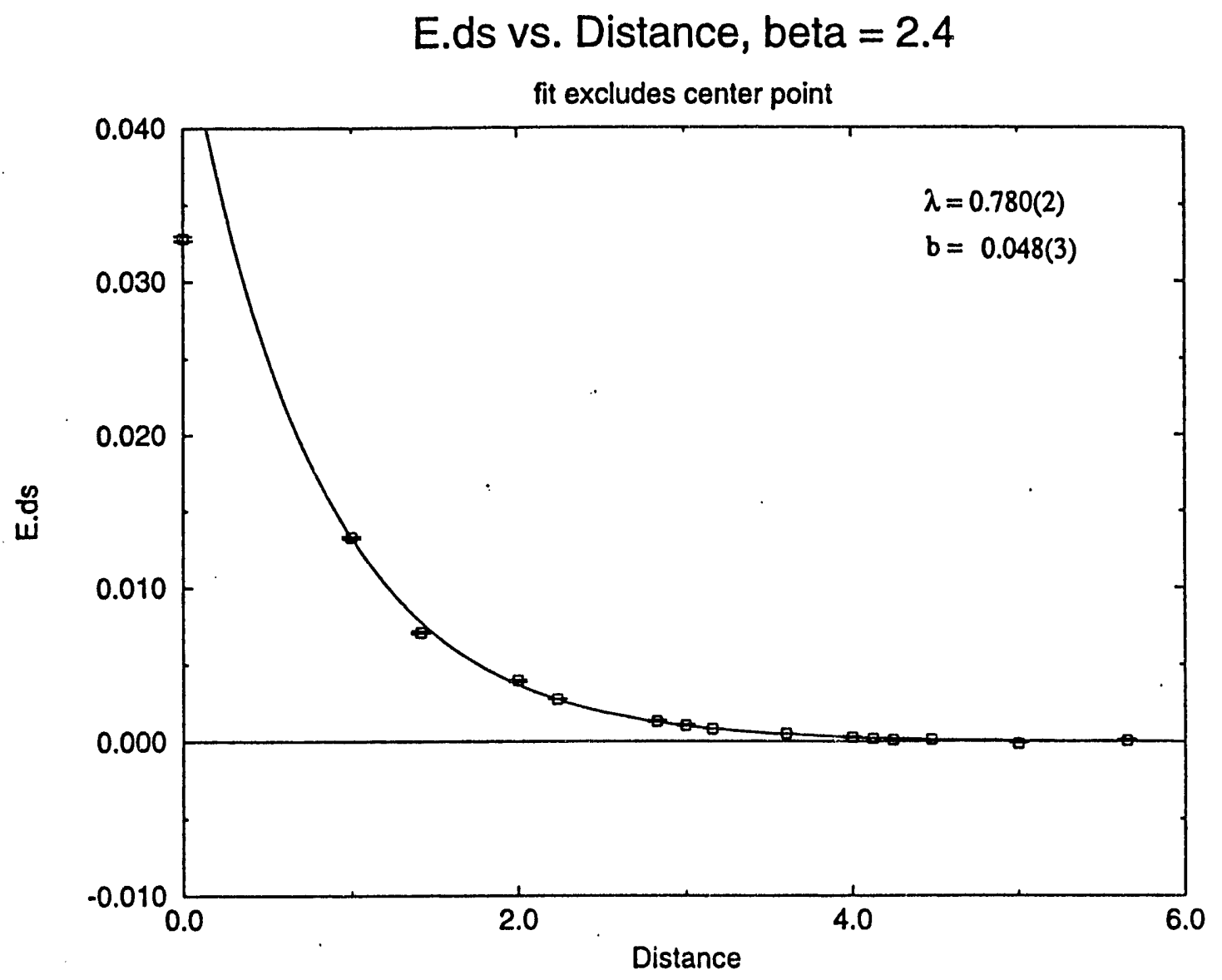

Figure 7: Behavior of the electric flux for gauge fixed $\mathrm{SU}(2)$ for $\beta=2.4$ ) as a function of the perpendicular distance $r_{\perp}$ from the $q \bar{q}$ axis. The solid line is an exponential fit to the data. 


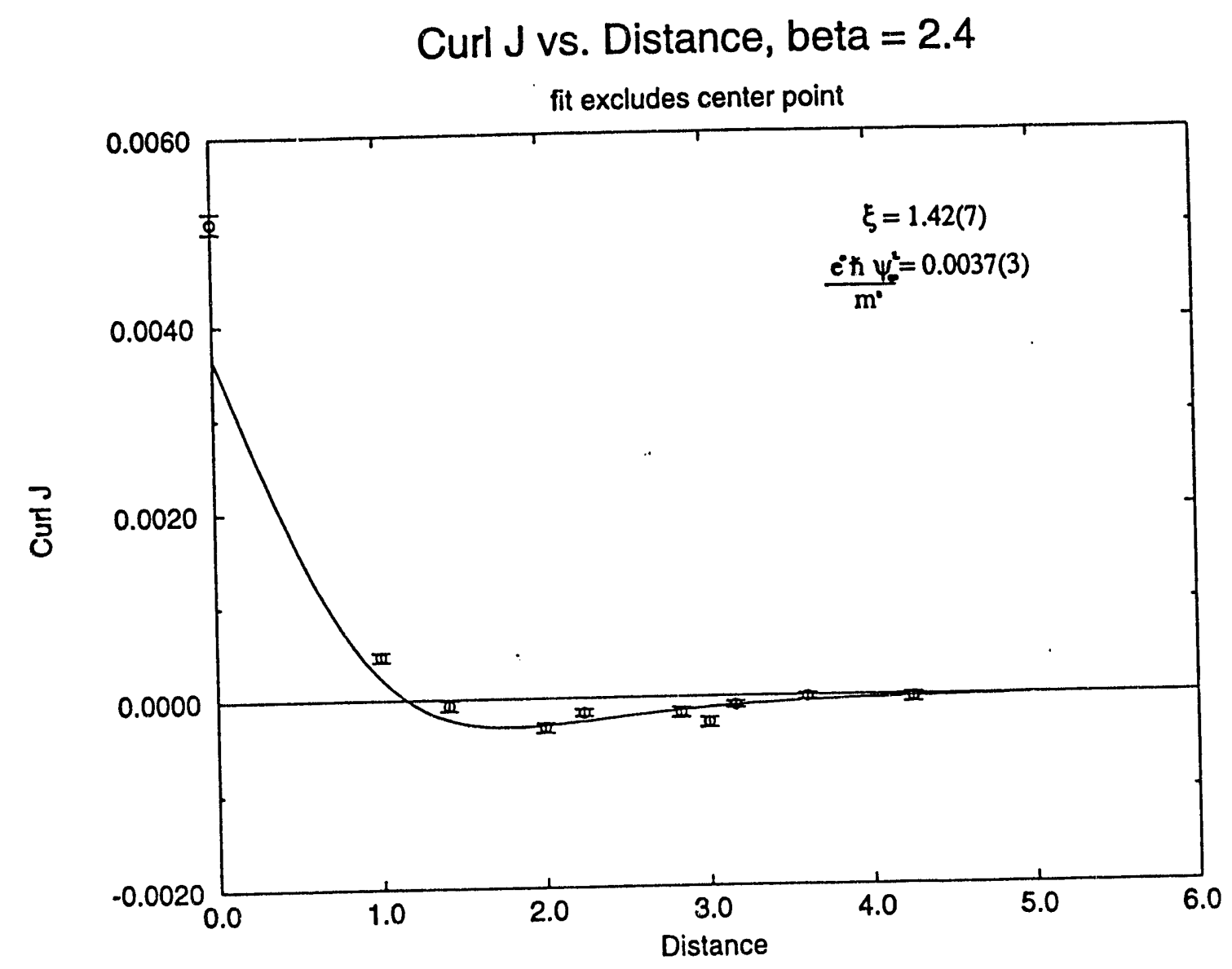

Figure 8: Behavior of the curl of the magnetic monopole current for gauge fixed $\mathrm{SU}(2)$ for $\beta=2.4$ ) as a function of the perpendicular distance $r_{\perp}$ from the $q \bar{q}$ axis. The solid line is the prediction of the Ginzburg-Landau theory with a coherence length $\xi$ shown. 


\section{(C)Lattice Gauge Theory (Ken Yee)}

In the current and near future I will be devoting my research efforts to three related issues in lattice gauge theory:

- (A)physical relevance (if any) of effective gauge boson masses recently computed in fixed-gauge lattice QCD and compact QED. Are these gauge-dependent masses indicative of an underlying dynamical Higgs mechanism in gauge theories? Is there an alternative (no-Higgs particle) explanation for electroweak boson masses even in the absence of fermions? Or are the observed gluon masses gaugefixing or lattice artifacts?

A hint was given in Refs. [1-5] where Bernard, Soni, and I showed that effective quark and gluon masses vary with gauge. This result was shown numerically on lattices with intermediate lattice spacing and analytically on infinite strong coupling lattices. It was also studied and confirmed in the Schwinger model. Nonetheless, since gauge dependence of quark and gl"on masses is such a fundamental issue, it is essential to understand this result at a deeper level.

Therefore, this summer at LSU I undertook a pedagogical study of the Landau gauge photon propagator in compact $\mathrm{QED}_{2+1}$, which is a QCD-like model with confinement, chiral symmetry breaking, and no phase transition in the continuum limit. In this model, I found that photons have a non-zero effective mass in Landau and Axial gauges, reminiscent of gluon effective masses in QCD [6]. Furthermore, I found that the photon mass varied substantially between Landau and Axial gauge.

Subsequently, I concentrated on answering the following question: What is the mechanism responsible for the gauge-dependence of photon mass in compact QED? To this end, I believe I have identified the mechanism for (a)generating photon masses and (b)making photon masses gauge dependent. This mechanism is chains of frustrated plaquettes, "Dirac strings."

The length and shape of Dirac strings are gauge variant. Some gauge conditions, such as lattice Landau gauge, can be satisfied only by relaxing to configurations with many Dirac strings; other gauge conditions tend to force all links to unity (or $\pi$ ) and so suppress string formation. Gauges which have more frustrated plaquettes have larger photon mass; gauges with fewer frustrated plaquettes have smaller photon mass. If one modifies $\mathrm{QED}_{2+1}$ by forbidding plaquettes (as done in Ref. [6]), one finds that the effective photon mass (in all gauges) nearly vanishes.

While there are clearly still many very interesting details and variations of this idea to be explored in QED, including the issue of whether photon masses are unphysical since Dirac strings are unphysical, the ultimate goal is to discover the analogous mechanism (if any) for gluon masses in QCD. In conjunction with 
this project, I have recently applied for a grant of supercomputer time at one of the DOE National Supercomputer Centers. While working mainly by myself, this project has already been and will be influenced by and complement the ongoing lattice study of the QCD vacuum at LSU (Browne, Haymaker, Singh), especially with regards to the relevance of magnetic monopoles and possible ways of defining them for nonabelian theories.

- (B)lattice gaugefixing as a technique for computing ultimately gauge invariant quantities in lattice QCD. This is a continuing project with Professor Claude Bernard at Washington University and Dr. Amarjit Soni at Brookhaven National Laboratory. One goal is to demonstrate convincingly that the Delta $I=1 / 2$ Rule can be computed from first principles in lattice QCD. This is a longstanding challenge which has remained unfulfilled despite attempts by leading lattice gauge theory groups on both sides of the Atlantic over the years. I will be sharing Bernard and Soni's computer-time at the National Supercomputer Centers for this project. (Summary in Refs. $[3,4]$ ).

- (C)analytical solution of lattice gauge theories. In Ref. [7] (which has recently been conditionally accepted by Rapid Communications), I extended Salmhofer's exact analytical solution of strong coupling $D=2$ QED with $r=1$ Wilson fermions on a square lattice to (i)all values of $r$ and (ii)parallelogram lattices. I am currently testing several ideas to further extend my solution to finite values of $\beta$. It is hoped that such solutions will eventually lead to a better understanding of Wilson fermions, the meaning of the Wilson $r$ parameter, and how lattice theories approach the continuum limit.

\section{References}

[1]K. Yee, "Gaugefixing Ambiguities and Gauge Dependence of Gauge Variant Schwinger Model Correlation Functions," Phys. Rev. D45 (1992) 4644

[2]K. Yee, "Gauge Dependence of Effective Quark Mass and Matrix Elements in Gaugefixed Large N Strong Coupling Lattice QCD," BNL preprint \#47712, submitted to Nucl. Phys. B[FS]

[3]C. Bernard, A. Soni, K. Yee, "Gaugefixed Lattice QCD: Masses and Matching Coefficients," Washington Univ. preprint HEP/92-33, to be submitted to Phys. Rev. D 
[4]C. Bernard, A. Soni, K. Yee, "Applications of Gauge-Fixed Correlation Functions of Quarks and Gluons," Nucl. Phys. B(Proc. Suppl.) 20 (1991) 410

[5]C. Bernard, A. Soni, K. Yee, "Introduction to Lattice Gaugefixing and Effective Quark and Gluon Masses," to be published in the Proceedings of the Workshop on QCD Vacuum Structure, Paris, France, 1992

[6]K. Yee, "Origin of Photon Mass in Compact $\mathrm{QED}_{2+1}$ : Monopoles and Dirac Strings," to be published in the Proceedings of Lattice '92, Amsterdam, The Netherlands, 1992

[7]K. Yee, "Central Charge of the Parallelogram Lattice Strong Coupling Schwinger Model," BNL preprint \#47604, conditionally accepted by Rapid Communications and under consideration for transfer to Phys. Rev. Lett. 


\section{EXPERIMENTAL - TASK B}

\section{A. Introduction}

The Louisiana State University experimental high energy group is now participating in electron proton colliding beam physics with the ZEUS experiment at DESY (Imlay, Metcalf, McNeil), in neutrino physics at Los Alamos National Laboratory (Imlay, Metcalf) and on various SSC activities. Svoboda joined the group two years ago and has been participating on the IMB experiment. He has recently joined the Dumand collaboration. This year $\mathrm{M}$. Cherry has joined the IMB collaboration.

LSU joined ZEUS in early 1989 and started working with other U.S. groups on the construction of the ZEUS barrel calorimeter. We undertook the task of preparing the wavelength shifter/light pipes for the electromagnetic part of the barrel calorimeter. Construction was completed in early 1991. We also contributed prominently to iests of some of these modules at Fermilab. Now we are participating on ZEUS data taking and analysis. Two papers on early physics results are in preparation.

At Los Alamos we completed data taking in 1989 for neutrino oscillation experiment E645. A paper has been submitted showing no evidence of oscillations for neutrinos from the decay at rest of $\pi^{+}$and $\mu^{+}$. In May of 1991 we joined LSND, a new neutrino experiment at LAMPF, which will have a greater sensitivity for neutrino oscillations and also will make other neutrino measurements, including vp elastic scattering. The experiment plans to start data taking in 1993.

The IMB detector turned off in April 1991 due to a major leak. Data analysis continues with $\mathrm{IMB}$ data. Recently, two papers were published on the subject of neutrino oscillations using measurements made of the background neutrino flux. In addition, there are several other papers now being worked on. Along with data analysis, a quarter-size IMB tank is being constructed in a test beam at the KEK facility in Japan. The tank will be used for various efficiency measurements and calibrations. These tests are being done in conjunction with the Kamiokande collaboration, who are providing the tank and site facilities. It is expected that the joint effort on the beam test will be followed by the construction of a very large water Cerenkov detector, SuperKamiokande. Both M. Cherry and R. Svoboda plan to participate in the SuperKamiokande experiment. 
The DUMAND construction effort at LSU is proceeding on schedule. Our effort is mainly in three areas, fabrication of titanium frames for optical modules, fabrication of the aluminum pressure housings for the underwater electronics, and organization of the off-line software group. The current plan is to have the first DUMAND string in the water by early next summer, to be followed three months later by two more strings.

Imlay and Metcalf are members of the SDC collaboration for SSC physics working on the scintillator calorimeter. Roger McNeil has joined the GEM collaboration and is performing very useful simulation studies for muon detection at the SSC.

LSU and Southern University are both members of the Particle Detector Research Center at Prairie View which is funded by the Texas National Research Laboratory Commission for SSC research. Southern works closely with LSU on The SDC collaboration. Dr. Fazely of Southern is also participating on the LSND experiment funded by a subgrant from LSU's DOE grant. 


\section{B . Neutrino Physics at LAMPF}

\section{Introduction}

The LSU group was a major participant on neutrino oscillation experiment E645 at LAMPF. Data taking was completed in 1989 and the analysis has now been completed. In 1991 we joined the LSND neutrino experiment at LAMPF, which we view as providing a natural continuation of our neutrino physics program. LSND will search for neutrino oscillations in the channels $v_{\mu} \rightarrow v_{e}$ and $\bar{v}_{\mu} \rightarrow \bar{v}_{e}$ with substantially greater sensitivity than E645. Several other neutrino measurements will also be made. In addition, if a neutrino beam upgrade is approved, we will measure neutrino-proton elastic scattering obtaining important information on the proton spin structure function and make a disappearance measurement of $v_{\mu}$, searching for $v_{\mu} \rightarrow v_{\tau}$ oscillations. LSND is scheduled to start data taking in 1993. LSU has primary responsibility for refurbishing the cosmic ray veto shield. LSU participants are R. Imlay, W. Metcalf, and R. Gunasingha. Ali Fazely of Southern University is also working on LSND.

\section{Experiment E645}

In 1980 LSU, OSU, ANL, and CIT proposed experiment E645 for LAMPF to search for neutrino oscillations in two channels $\bar{v}_{\mu} \rightarrow \bar{v}_{e}$ and $v_{e} \rightarrow \bar{v}_{e}$. Our neutrino source consists of an equal admixture of $v_{\mu}, \bar{v}_{\mu}$, and $v_{e}$ arising from decays at rest of $\pi^{+}$and $\mu+$ in a beam stop. The appearance of $\bar{v}_{\mathrm{e}}$ in our detector would indicate the occurrence of neutrino oscillations. The experiment took data for three years $(1987,1988$ and 1989). Results from the 1987 data have been published ${ }^{1}$ A paper containing results for the entire run has accepted for publication in the Physical Review2. We see no evidence for neutrino oscillations in the decay at rest sample.

Although we have concentrated on the study of the decay at rest neutrino sample one can also look for neutrinos above $60 \mathrm{MeV}$ in the detector. This high energy sample can be used to set limits on various exotic processes such as $\pi^{\circ} \rightarrow v \bar{v}$ and $\eta \rightarrow v \bar{v}$. It can also be used to set limits on $v_{\mu} \rightarrow v_{e}$ oscillations for $v_{\mu}$ arising from the decay in flight of $\pi^{+}$in the beam stop. In fact one of the primary goals of LSND will be to search for $v_{\mu} \rightarrow v_{e}$ oscillations with $v_{\mu}$ from decay in flight $\pi^{+}$. 
Ali Fazely of LSU made an analysis of the sample of E645 events above $60 \mathrm{MeV}$. The decays $\pi^{\circ} \rightarrow V \bar{v}$ and $\eta \rightarrow v \bar{v}$ are forbidden in the standard picture where $m_{v}=0$ and lepton number is conserved. Ali obtains preliminary limits on the branching ratios, $B R\left(\pi^{\circ} \rightarrow v \bar{v}\right) \leq 5 \times 10^{-7}$ and $B R(\eta \rightarrow v \bar{v}) \leq 10^{-5}$. For comparison a recently published limit is $B R\left(\pi^{0} \rightarrow v_{e} \bar{v}_{e}\right)<8.3 \times 10^{-7}$.

If the $v_{\mu}$ from $\pi^{+}$decay in flight has enough energy it can produce a $\mu^{+}$in our detector which will stop and decay to an electron. A few of thes nuons will have enough energy to trigger. More generally we will trigger on the electron and see the muon several microseconds in the past. These events can not be completely separated from events produced by cosmic ray muons but, as expected, a clear beam excess ( 40 events) is observed. The observed rate is consistent with but slightly lower than that given by a calculation made by Burman et al. for the LSND experiment. This is in conflict with the recently published results of another LAMPF experiment that quotes a higher than expected $v_{\mu} C^{12}$ cross section ${ }^{3}$. Ref. 3 also sees an unexpectedly low muon energy spectrum while we do not. Both the $v_{\mu}$ flux and $v_{\mu} \mathrm{C}^{12}$ cross section have strong energy dependences so that the event rate is difficult to estimate. The enhanced sensitivity of LSND should allow it to make a more accurate measurement of this cross section which is crucial to many low energy neutrino measurements.

The high energy decay in flight $v_{\mu}$ can also be used for a neutrino oscillation experiment looking for $v_{\mu} \rightarrow v_{e}$. The cross section for $v_{e}$ will be much higher than for $v_{\mu}$. Because the electrons are much higher in energy than electrons from muon decay selection cuts designed to remove cosmic ray muons can be largely eliminated. A search of the E645 data produced 21 beam on events with a beam excess of 10 events. Unfortunately, this is only a two sigma enhancement above the expected background so we cannot draw any definite conclusions from it. However, it is a tantalizing example of the possible physics LSND may uncover.

The LSU-Southern group has spent a good part of the past year preparing the decay-at-rest result for publication and working on the above decay-in-flight issues for the benefit of LSND.

\section{LSND Experiment, E1173}

\section{a) Physics Goals}

The Liquid Scintillator Neutrino Detector (LSND) at LAMPF shown in Figure 1 is presently nearing completion. The major goals given in the proposal are to search for $v_{\mu} \rightarrow v_{e}$ and $\bar{v}_{\mu} \rightarrow \bar{v}_{e}$ oscillations with high sensitivity using $v_{\mu}$ from $\pi^{+}$decay in flight and $\bar{v}_{\mu}$ 


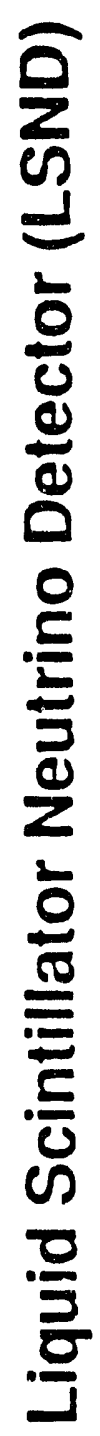
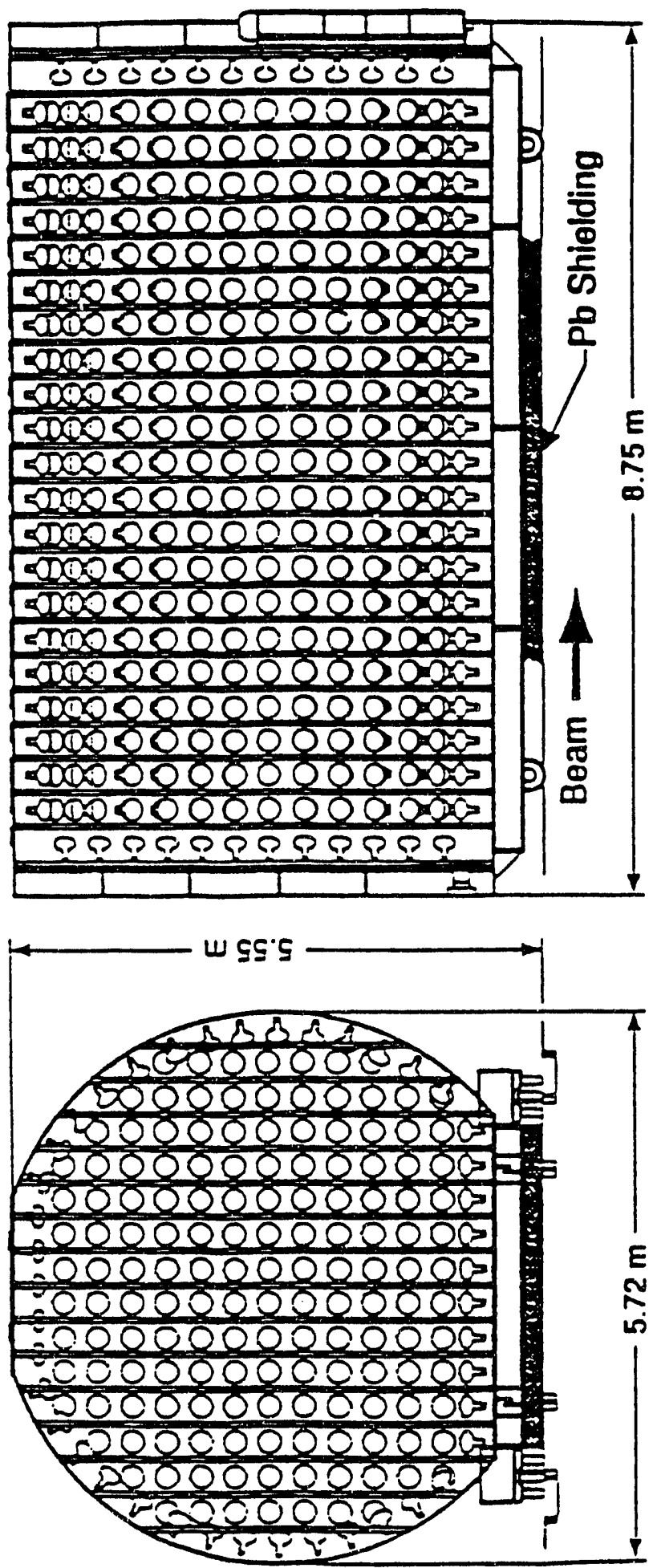

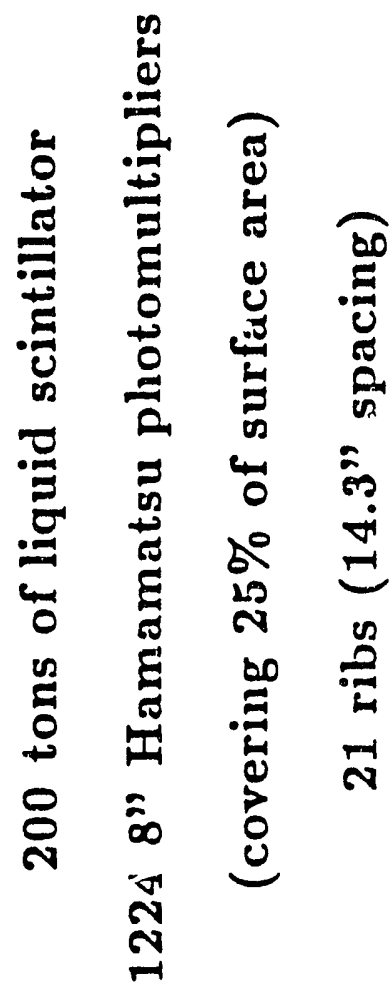

$\sum_{E=1}^{\infty}$ 
from $\mu^{+}$decay at rest. Figure 2 shows the two-parameter space of $\Delta \mathrm{m}^{2} \equiv \mathrm{m}_{1}^{2}-\mathrm{m}_{2}^{2}\left(\mathrm{eV}^{2}\right)$ and $\sin ^{2} 2 \theta$ that customarily describe neutrino oscillations. Plotted are (i) the $90 \%$ confidence level oscillation limits from the reactor (Gosgen) ${ }^{4}$ disappearance $\left(\bar{v}_{e} \rightarrow \bar{v}_{x}\right)$ experiments, (ii) the BNL (E734) ${ }^{5}$ limiting curve for $v_{\mu} \rightarrow v_{e}$, (iii) the LAMPF (E645) ${ }^{1}$ limit for $\bar{v}_{\mu} \rightarrow \bar{v}_{e}$, and (iv) the LAMPF (E764) 6 limit for $v_{\mu} \rightarrow v_{e}$. Recent results of BNL (E776) ${ }^{7}$ are not shown. Also indicated in Figure 2 are the limiting curves expected from LSND's two independent oscillation searches. After two years of data collection a limit on $\sin ^{2} 2 \theta$ of approximately $3 \times 10^{-4}$ for all $\Delta \mathrm{m}^{2}>1 \mathrm{eV}^{2}$ can be achieved, and a limit on $\Delta \mathrm{m}^{2}$ of approximately $1.7 \times 10^{-2} \mathrm{eV}^{2}$ will be obtained for $\sin ^{2} 2 \theta=1$.

There are many other physics objectives that can be pursued with the liquid scintillator detector. The LSND data acquisition system (DAQ) will have the capability of triggering on hundreds of events per second and transferring up to $4 \mathrm{MB} / \mathrm{s}$ of data to a farm of UNIX workstations, where the events will be fully reconstructed, so that many different physics topics can be pursued simultaneously. Searching for $\bar{v}_{\mu} \rightarrow \bar{v}_{e}$ oscillations is equivalent to searching for the lepton number violating decay $\mu^{+} \rightarrow \mathrm{e}^{+} v_{\mu} \bar{v}_{\mathrm{e}}$. Limits on this decay are directly comparable to limits on neutrino oscillations, so that we should be sensitive to branching ratios as low as $10^{-4}$. The $v C \rightarrow v C^{*}$ neutral-current reaction, one of the only neutrino-nuclear neutral-current reactions that can be easily observed, will be measured to approximately $10 \%$ accuracy and will be recognized by the detection of the $15.11-\mathrm{MeV} \gamma$ emitted by the excited carbon nucleus when it decays to the ground state. The KARMAN collaboration has measured this reaction with a $50 \%$ uncertainty. ${ }^{8}$ The rare decays $\pi^{\circ} \rightarrow v \bar{v}$ and $\eta \rightarrow v \bar{v}$, followed by $v_{e} C \rightarrow e-N$, can be searched to sensitivities of about $10^{-8}$ and $10^{-4}$, respectively, with very little background because the neutrinos from these decays are extremely energetic. These decays are forbidden for massless Weyl neutrinos and can proceed only if neutrino states of both chiralities exist or if lepton number is not conserved. ${ }^{7}$ We shall also measure the $v_{e} \mathrm{C}$ and $v_{\mu} \mathrm{C}$ charged-current scattering cross sections and the ve elastic scattering cross section to approximately $10-15 \%$ accuracy. These measurements should test present theories of neutrino-nucleus scattering and provide a measurement of $\sin ^{2} \theta_{W}$, the fundamental parameter of the standard model of electroweak interactions. Finally, we will obtain a large sample of neutrino-proton elastic-scattering events, where the neutrinos are from pion decay in flight. A beam stop upgrade to enhance pion decay in flight has been proposed. This is discussed in section c. The upgrade would allow a much improved measurement of vp elastic scattering which can be used to determine the strange quark contribution, $\Delta s$, to the spin of the proton. The upgrade would also allow a search for $v_{\mu} \rightarrow v_{\tau}$ in a $v_{\mu}$ disappearance experiment. 


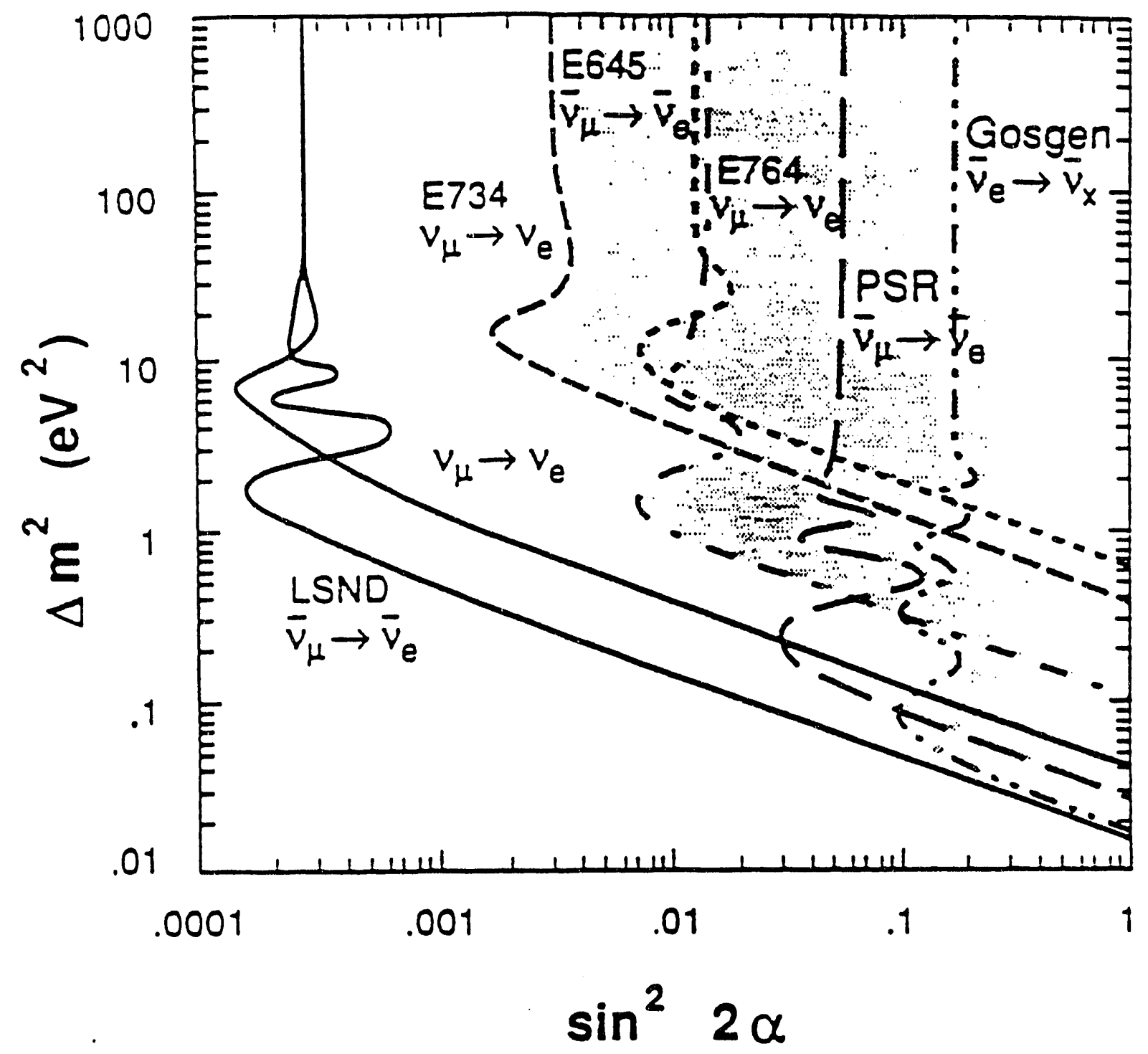

The present $\bar{\nu}_{\mu} \rightarrow \bar{\nu}_{e}$ oscillation limits from reactor (Gosgen), BNL (ET34), and LA.MPF (E645, ET64) experiments expressed in terms of the $\Delta m^{2}$ vs. $\sin ^{2} 2 \theta$ twoparameter space. Also shown are the limiting curres expected from ISND with neutrinos from $\mu^{+}$decay at rest and $\pi^{+}$decay in fight.

Figure 2 


\section{b) Detector and Beam}

The detector, as shown in Figure 1, consists of a cylindrical tank of dilute mineral-oilbased liquid scintillator approximately $6 \mathrm{~m}$ in diameter by $9 \mathrm{~m}$ long with an active mass of 200 tons. The tank will reside inside the existing E645 veto shield, which will be located $28 \mathrm{~m}$ downstream of the proton beam stop at an angle of approximately $12^{\circ}$ to the beam direction. The proton kinetic energy entering the beam stop is $760 \mathrm{MeV}$ and the typical proton current is $800 \mu \mathrm{A}$. The beam stop area consists of a 20 -cm-long water production target followed by an array of isotope-production target stringers and a water-cooled $\mathrm{Cu}$ beam dump. There exists about $8.5 \mathrm{~m}$ of Fe-equivalent between the $\mathrm{Cu}$ beam dump and the detector. In this present arrangement, approximately $97.5 \%$ of the $\pi^{+}$produced in the beam stop decay at rest and emit $30-\mathrm{MeV}$ muonneutrinos, while $2.5 \%$ of the pions decay in flight and create a muon-neutrino beam with an average energy of about $150 \mathrm{MeV}$.

The detector tank is made of 1-cm-thick steel and has 12248 -inch diameter photomultiplier tubes mounted uniformly over the inside surface ( $25 \%$ coverage). These tubes are manufactured by Hamamatsu (R1408) and have good timing resolution, 2.8-ns FWHM for single photons and full-face illumination. They also have good single photoelectron separation and typical noise rates $<5 \mathrm{kHz}$. We will be using a new idea in which the scintillator is deliberately diluted such that about $20 \%$ of the total light output will be Cerenkov light, $80 \%$ scintillation light, and 20 photoelectrons will be observed per MeV of deposited energy. The Cerenkov light will enable us to distinguish electrons from protons and to reconstruct the directions of electrons. The liquid scintillator consists mostly of mineral oil (>99.99\%) with the addition of about $0.03 \mathrm{~g} / \mathrm{l}$ of b-PBD. Mineral oil has advantages relative to water for the detection of Cerenkov light as it has a higher index of refraction, a lower density so that electrons travel farther before stopping, and a sharper Cerenkov ring due to the longer radiation length.

Electrons and protons have been reconstructed by a Monte Carlo simulation of the detector. The simulation is based on EGS4 and includes scintillator absorption, Rayleigh scattering, reflections off phototube surfaces, and phototube noise counts. For $45-\mathrm{MeV}$ protons produced inside the detector, we expect an average reconstructed position error of $10 \mathrm{~cm}$, an average reconstructed time error of $0.5 \mathrm{~ns}$, and an energy resolution of $5 \%$. For $45-\mathrm{MeV}$ electrons produced inside the detector, we expect the same spatial, time, and energy resolutions, and an average directional error of $12^{\circ}$. 
The liquid scintillator we plan to use in LSND consists of mineral oil with $0.03 \mathrm{~g} /$ of $\mathrm{b}$ PBD. The mineral oil comes from Petroleum Specialties International (BRITOL 6NF HP) and has an attenuation length which increases from $20 \mathrm{~m}$ at a wavelength of $400 \mathrm{~nm}$ to $50 \mathrm{~m}$ at a $500 \mathrm{~nm}$ wavelength. The mineral oil is also very low in radioactivity and has a Th content of less than one part per trillion. The b-PBD scintillator additive has the advantages of a low wavelength absorption band $(\lambda<360 \mathrm{~nm})$, a short fluorescence lifetime ( $\tau=1.2 \mathrm{~ns})$, and a fluorescence emission maximum $(\lambda=365 \mathrm{~nm})$ which matches well the response of our Hamamatsu phototubes.

A series of measurements were performed in the LAMPF test beam during 1990-1991 to determine the properties of our liquid scintillator mixture. A $1.5-\mathrm{m}$ long, 5-cm diameter PVC pipe was filled with different liquids and placed in the test beam. A 2 " phototube with a photocathode response similar to the 8 " Hamamatsu tubes was mounted at one end of the pipe and at a distance of $1 \mathrm{~m}$ from the beam crossing point. The angle of the pipe with respect to the beam was varied from 25 to $90^{\circ}$. Figure 3 is a histogram of the relative number of photoelectrons measured in the phototube as a function of the angle with respect to a beam of $100 \mathrm{MeV} / \mathrm{c}$ positrons for pure mineral oil and mineral oil with $0.25 \mathrm{~g} / \mathrm{l}$ of $\mathrm{b}-\mathrm{PBD}$. With pure mineral oil there is a pronounced peak at the $47^{\circ}$ Cerenkov angle and very little light outside the peak, while with the b-PBD mixture there is the Cerenkov peak and a flat distribution of scintillation light. The Cerenkov peaks in the two plots are almost identical, which shows that very little Cerenkov light $(<15 \%)$ is lost by adding the b-PBD. It was also confirmed that the proton scintillation light time distribution has a larger slow component than the positron light. This pulse shape discrimination (PSD) will provide a way to perform particle identification in addition to fitting the Cerenkov cone.

LSND is on schedule for the goal of completion by March, 1993. The tank is complete, almost all of the phototubes have been delivered, shield refurbishing will be finished by November and the electronics and DAQ systems are designed and under construction.

\section{c) Enhanced Decay in Flight Upgrade}

LSND has proposed that the LAMPF A-6 beam stop facility be modified in order to enhance the flux of muon-neutrinos at the detector location. We present here an abbreviated version of the LSND report. It is estimated that a factor of approximately 5 in the decay-in-flight (DIF) neutrino flux can be gained through design changes that are practical at moderate cost. The proposed design incorporates a radiation-hardened dipole magnet to bend the proton beam $9^{\circ}$ toward the detector location, a $30-\mathrm{cm}$-long water target for the production of pions by the proton 


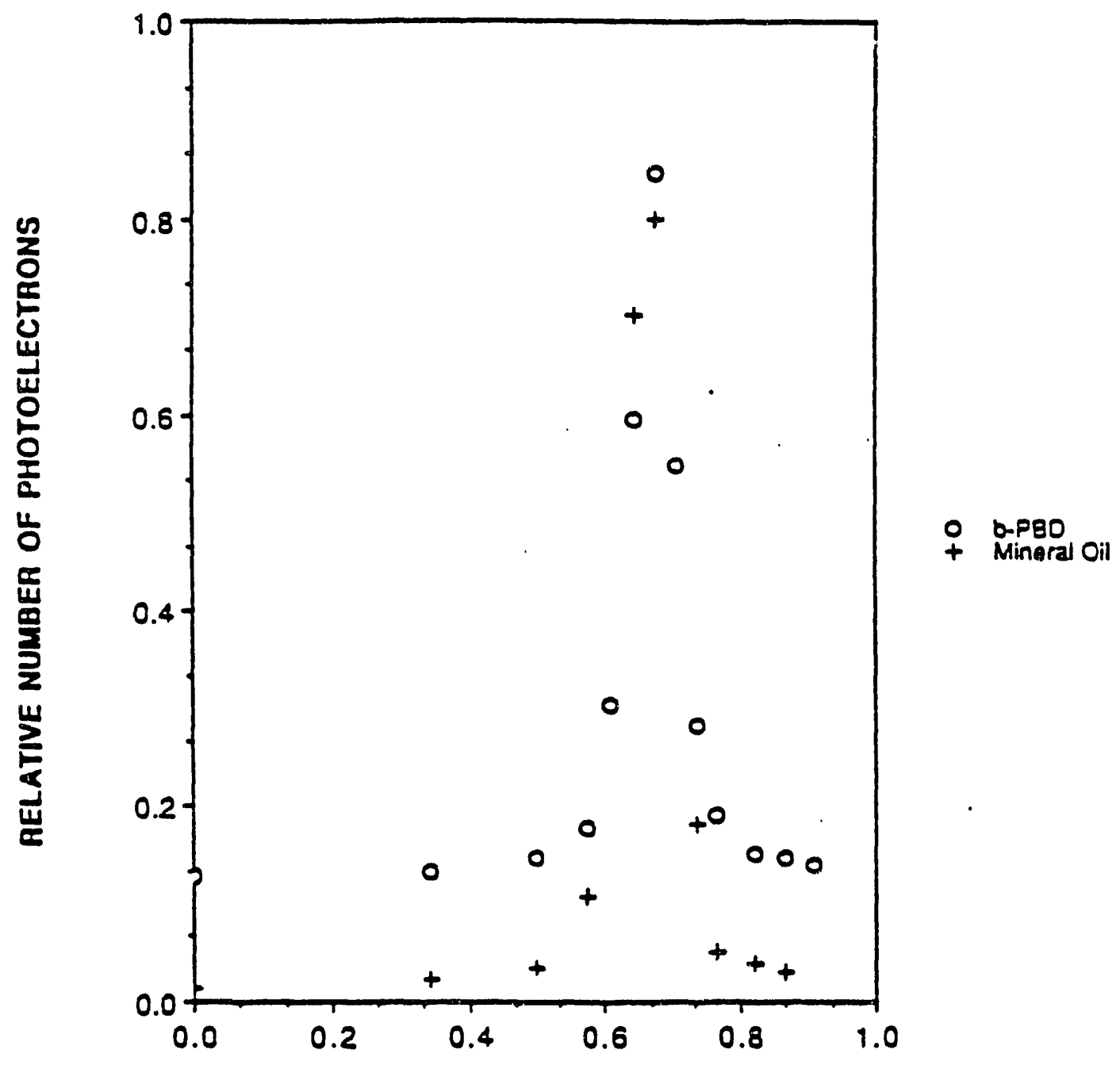

COS(THETA)

Relative number of photoelectrons vs. the angle of the test cylinder with respect to the positron beam for pure mineral oil and mineral oil with $0.25 \mathrm{~g} / \ell$ of b-PBD scintillator.

Figure 3 
beam, and a $\sim 1.5$-m channel for the pions to decay. These changes can be carried out with components of proven design using LAMPF's existing remote-handling techniques. No interruption of the annual running schedule will be necessary if priority is assigned for hardware instrallation during a routine shutdown period. A preliminary estimate of this project gives a visible cost of $\$ 1.15 \mathrm{M}$ plus 11 man-years of effort, including engineering, design, fabrication, and installation.

The upgrade is motivated primarily by two physics topics. These are a study of neutrinoproton elastic scattering aimed at measuring the strange quark contribution to the spin of the proton, and a search for neutrino oscillations of the type $v_{\mu} \leftrightarrow v_{\tau}$ by a precision measurement of the disappearance of $v_{\mu}$ (and $\bar{v}_{\mu}$ ) from the essentially pure $v_{\mu}$ (and $\bar{v}_{\mu}$ ) upgraded decay-in-flight beam.

The only two observables in $v p \rightarrow v p$ are the recoil proton kinetic energy and the angle of the recoil proton with respect to the incident neutrino. (We assume that the recoil neutrino and the recoil proton polarization are not measurable in a practical way.) The proton kinetic energy is given by $T_{p}=Q^{2} / 2 m_{p}$, where $Q^{2}$ is the momentum transfer squared of the reaction and $m_{p}$ is the proton mass. The angle of the recoil proton in the lab frame can be expressed as $\cos \theta=\left(1+m_{p} / E_{v}\right) /\left(1+2 m_{p} / T_{p}\right)^{1 / 2}$, where $E_{v}$ is the energy of the incident neutrino. In LSND we are unable to measure the proton angle, so that the proton kinetic energy, $T_{p}$, is the only signature.

The issue of the contribution of strange quarks to the proton spin has surfaced in recent and very difficult experiments of the deep inelastic scattering of polarized muons from polarized protons. The sensitivity to the proton spin in that case is achieved by measuring the yield as a function of the spin direction. In our case, the sensitivity to the proton spin is a direct consequence of the weak axial coupling of the proton, and as vp $\rightarrow$ vp does not require the constituents to

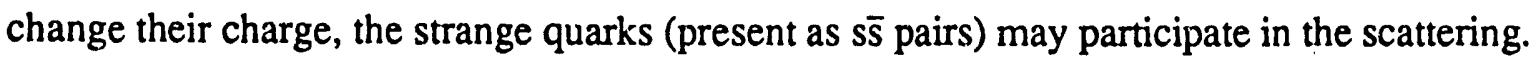

We calculate the number of events per year based on 130 days of running or about 3000 hours of actual beam. With the upgraded neutrino beam, and assuming a $10-\mathrm{MeV}$ recoil proton threshold (see Figure 4), we anticipate the order of $10,000 \mathrm{vp} \rightarrow$ vp events per year. The main background is $\mathrm{VC} \rightarrow \mathrm{pX}$, neutrino scattering off a bound proton, which is estimated to occur at a rate at least comparable to $v p \rightarrow v p$. We are investigating a variety of ways of directly dealing with this background and can, if necessary, subtract it by taking data with a scintillator with a different $\mathrm{C} / \mathrm{H}$ ratio. 


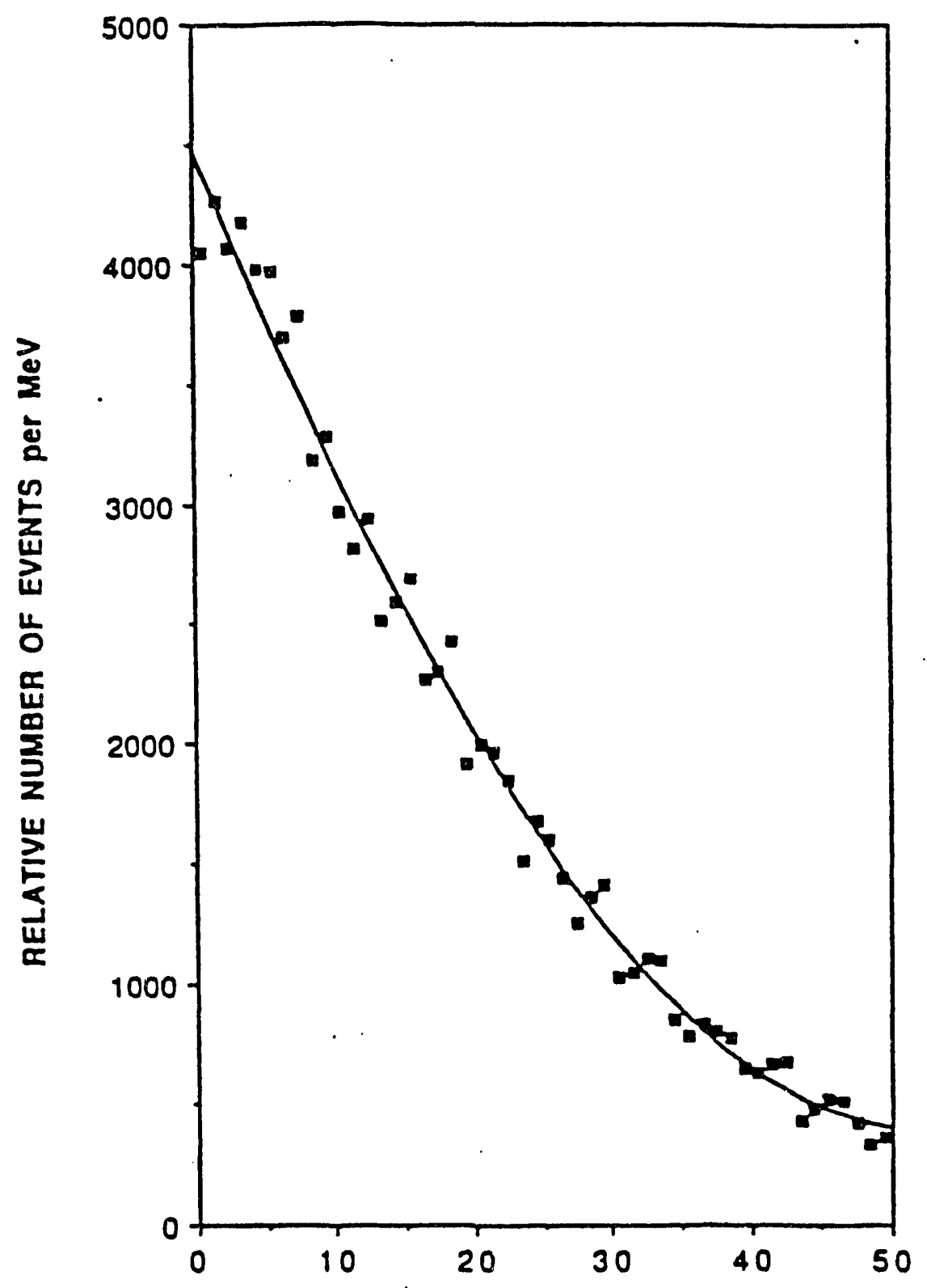

PROTON KINETIC ENERGY (MEV)

The recoil proton kinetic energy distribution from $\nu p \rightarrow \nu p$ after folding in the LAMPF neutrino energy spectrum.

Figure 4 
The projected sensitivity for measuring the $v p \rightarrow v p$ cross section after two years of data collection yields a total error on the cross section estimated to be $\pm 7 \%$, corresponding to a \pm 0.04 error on $\Delta s$, i.e., a factor of 2 improvement on the only previous measurement ${ }^{8}$ as well as a more valid interpretation because of a lower $Q^{2}$ than that measurement. Such a measurement will be complementary to future polarized DIS experiments: the SMC experiment at CERN and HERMES experiment at DESY.

Second, a beam upgrade would allow a search for neutrino oscillations of the type $v_{\mu} \leftrightarrow v_{\tau}$. This would be done by means of a precision measurement of the disappearance of $v_{\mu}\left(\right.$ and $\bar{v}_{\mu}$ ) from a LAMPF pion decay-in-flight neutrino beam that is essentially pure in $v_{\mu}\left(\right.$ and $\left.\bar{v}_{\mu}\right)$. Such a measurement can only be done with the upgraded neutrino beam. The motivation for the $v_{\mu} \leftrightarrow v_{\tau}$ search arises in part from results on solar neutrinos from the Kamiokande-II detector, the ${ }^{37} \mathrm{Cl}$ Solar neutrino detector and the SAGE and GALEX detectors. The results suggest, but do not compel one to believe, that resonant neutrino oscillations occur in the matter of the Sun. This in turn suggests that the masses of $v_{e}$ and $v_{\mu}$ are different from zero, but each is appreciably less than $1 \mathrm{eV} / \mathrm{c}^{2}$, and that the mixing strength between $v_{\mathrm{e}}$ and $v_{\mu}$, namely $\sin ^{2} 2 \theta_{\mathrm{e} \mu}$, is roughly $10^{-2}$.

Motivation for a search at LAMPF lies in recognition of the fact that the $v_{\mu}$ flux from the decay-in-flight beam at LAMPF, when weighted by the cross section for $v_{\mu} \rightarrow \mu$ (specifically by $\sigma\left(v_{\mu}+{ }^{12} \mathrm{C} \rightarrow \mu^{-}+{ }^{12} \mathrm{~N}\right)$ ), peaks in the vicinity of $175 \mathrm{MeV}$. Hence, the wavelength of $v_{\mu} \leftrightarrow v_{\tau}$ oscillations, $\lambda_{\mu \tau}=2.47 \mathrm{E}_{\mathrm{v}}$ ( in $\left.\mathrm{MeV}\right) / \Delta \mathrm{m}_{\mu \tau}^{2}$ (in eV2), would be, for example, $4.3 \mathrm{~m}$ for $\mathrm{m}\left(v_{\mu}\right)<1$ $\mathrm{eV} / \mathrm{c}^{2}$ and $\mathrm{m}\left(v_{\tau}\right) \simeq 10 \mathrm{eV} / \mathrm{c}^{2}$. For these or similar mass values and $\sin ^{2} 2 \theta_{\mu \tau}$ of the order of $10^{-2}$ or larger, it is possible to observe $v_{\mu} \leftrightarrow v_{\tau}$ oscillations directly in the 9-m-long LSND detector in a self-normalizing experiment. The $v_{\mu}$ disappearance experiment proposed here seeks to measure a modulation of the order of $1 \%$ of an otherwise uniform signal along the length of LSND.

Consequently, the desired statistical precision necessitates an event sample of $>10^{5}$ events using LSND in an improved DIF neutrino beam at LAMPF. It is necessary to provide an additional signal -independent of neutrino flavor - to measure precisely variations in detection efficiency with position and angle that may be inherent in the detector itself. This normalizing reaction will be $v+p \rightarrow v+p$, the properties of which are the same for all neutrino types. The principal requirements for a sensitive search are cleanliness and abundance of the $v_{\mu}$ signal reaction, and an abundant flavor-independent neutrino reaction $: n$ measure precisely the neutrino detection 
efficiency over the detector volume. Both requirements can be met by LSND exposed to an improved DIF neutrino beam at LAMPF.

We believe that a sensitive search for $v_{\mu} \leftrightarrow v_{\tau}$ in the region $1 \leq \Delta \mathrm{m}_{\mu \tau}^{2} \leq 400 \mathrm{eV}^{2}$ and $5 \mathrm{x}$ $10^{-3} \leq \sin ^{2} 2 \theta_{\mu \tau} \leq 1$ is possible in a $v_{\mu}$ disappearance experiment using LSND as planned and now being constructed. This region is shown as the cross hatched area in Figure 5. There exists at present a result that extends below the value $\sin ^{2} 2 \theta_{\mu \tau}=0.1$ from only a single search for $v_{\mu}$ and $v_{\tau}$ oscillations; 9 the region excluded by that search is also shown in Figure 5.

The overlap in Figure 5 of the regions explored by the search proposed here and the previous search is advantageous for two reasons. First, it is, as usual with experimental results, desirable to confirm the result of the previous search, the data for which stand alone in contrast to the many (also) negative results for $v_{\mu} \leftrightarrow v_{e}$ oscillations. Second, the previous search involved an appearance experiment in which the production and decay of charged tau leptons in a high energy ( $\geq 10 \mathrm{GeV}$ ) admixed $v_{\mu}$ and $v_{\tau}$ beam were searched for, and none found and thus is complimentary to the search proposed here.

\section{d) Veto Shield-LSU Contribution}

LSND will be located in the same shielded tunnel as was used by E645 and will use the same cylindrical liquid scintillator veto shield in a slightly modified form. The active veto shield and the $2000 \mathrm{gm} / \mathrm{cm}^{2}$ of passive shielding are necessary to reduce cosmic ray muon and neutron backgrounds to an acceptable level. LSND anticipates adding $1,000 \mathrm{gm} / \mathrm{cm}^{2}$ of passive shielding. The active shield has been rotated to interchange the upstream and downstream ends. Also we will not have a veto counter under the detector. The active shield has continuous coverage on the cylindrical surface and two ends of the cylinder except for a minor crack, now located along the circumference of the upstream end. The optics of the shield is also not as good at the boundary forming the crack.

In 1990 LSND personnel opened up and examined the mounting for about 40 PMTs on the shield. They concluded that there should be a general refurbishing using a modified mounting scheme. When the LSU group joined LSND in May 1991 we agreed to take substantial responsibility for refurbishing the shield. LSU also agreed to make new bases so that the shield can use the same electronics and cabling as will be used for the PMTs on the main detector. Finally, we have agreed to cover the crack with plastic scintillation counters. 


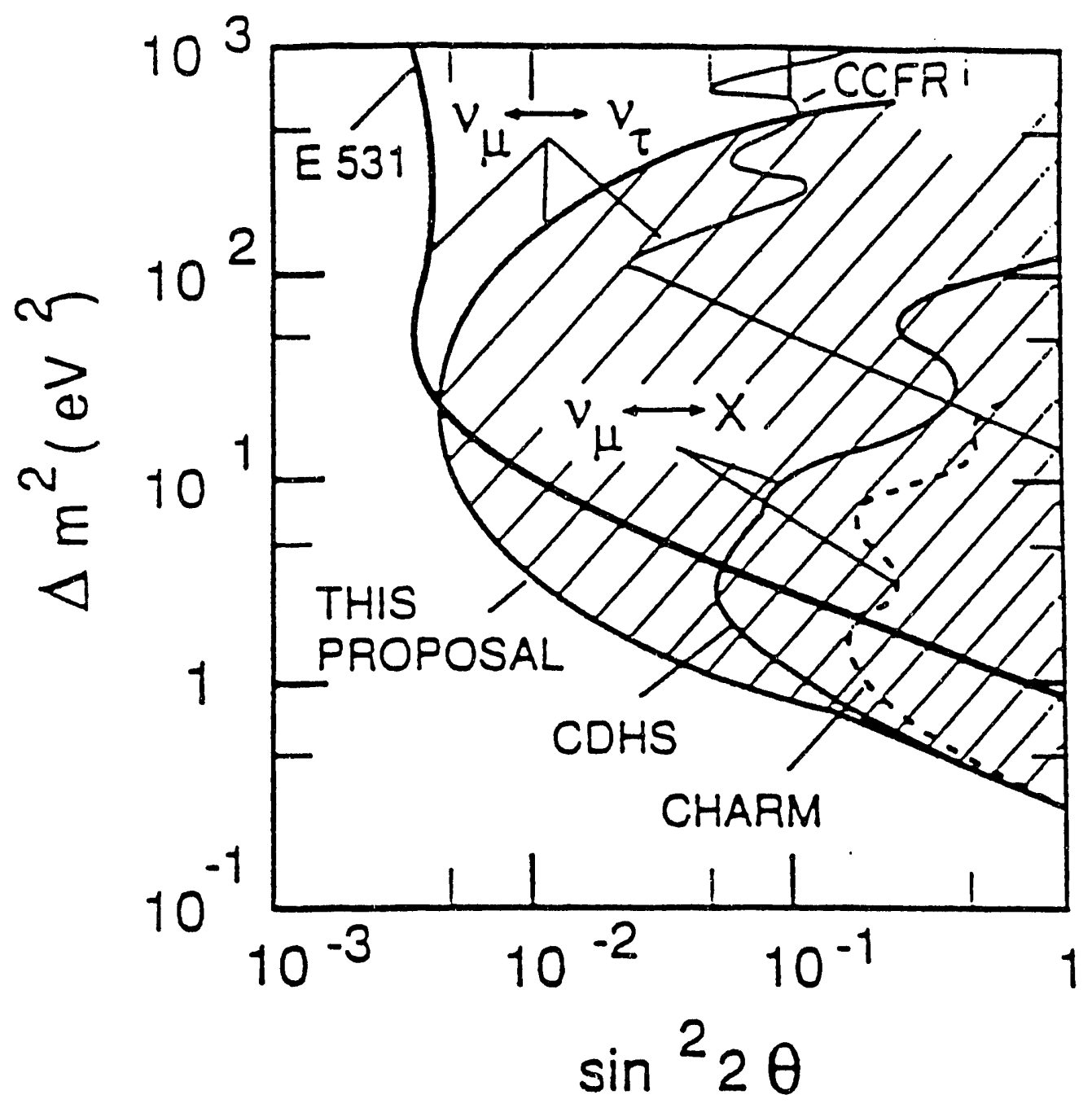

Plot of $\Delta m_{\mu r}^{2}$ vs. $\sin ^{2} 2 \theta_{\mu r}$ showing results of earlier experiments and the anticipated result of this proposal.

Figure 5 
Aside from providing E645 results of importance to LSND, the bulk of LSU's neutrino work this past year has been fulfilling the above commitments. We expect this work to be nearly complete by the end of the year. In addition to faculty members from LSU and Southern we have employed (at LAMPF) a part-time technician, Bill Marterer, who previously worked for LSU but now resides in Santa Fe. A full-time LSU technician, Gary McMills, has contributed to this work both here and at LAMPF. At LAMPF we have also received valuable help from three summer students from Southern University, two technicians from the UCSB and various LAMPF personnel. At LSU we have been aided by several student workers.

By January 1992 we had completed removing all the tubes from the shield. The bulk of the work was done by Gary McMills with the help of LAMPF people. The metal parts were shipped to LSU for cleaning (by Gary and student workers). Then the LSU shop machined the main mounting rings to ensure a better fit on re-assembly. At LAMPF, Bill Marterer with help from various sources noted above, cleaned all 292 mounting flanges on the shield to ensure they were rust free and ready for remounting. He also supervised the design and procurement of the new gaskets that will be used to give a better seal. By early June the cleaning was complete and all parts had been returned to LAMPF. Bill Marterer, with the aid of the summer students, then began to remount the tubes on the upstream wall of the shield. This was followed by extensive tests to ensure the wall had neither light nor liquid leaks. In early August we began to remount the tubes on the rest of the shield. This work has been slowed by the loss of the summer students but the LSU thesis student, R. Gunasingha, will arrive in mid-September to help and we anticipate this job should be complete by early November. We can then refill the shield with scintillator as soon as the required containment dam is completed and begin to actually tune the individual tubes. This work will be largely the responsibility of Mr. Gunasingha assisted by faculty from LSU and Southern.

In the spring of 1992 the MP-4 division at LAMPF agreed to provide plastic scintillator (from existing stocks) for twenty $30 \times 40 \times 1$ inch counters to cover the crack between the upstream wall and the rest of the shield. They also agreed to purchase lucite light guides. The scintillator was shipped to LSU where it was cut to the proper sizes and polished. The scintillator shop at MP-4 has done much of the light guide preparation. The phototubes come from those used by E645. (We are mounting light guides and phototubes on both ends of each counter to ensure maximum efficiency and to give redundancy since it will be very difficult to replace tubes once the shield is in place). Gary McMills is supervising the final assembly of the counters and we expect to finish all twenty by October. Mr. Gunasingha is testing them as they are completed using a 


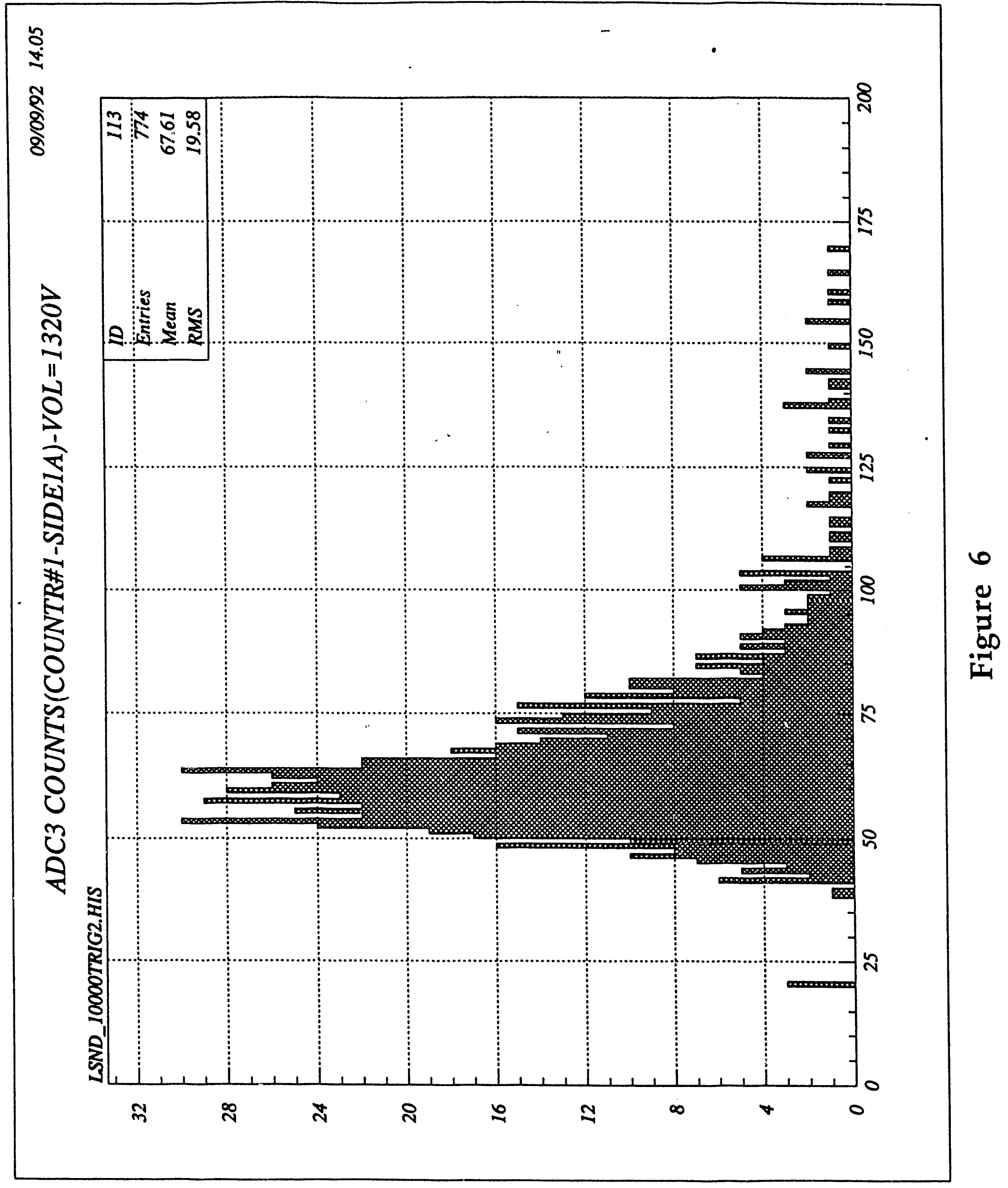


cosmic ray test stand that our group has built using money provided by a grant from the state of Louisiana. Fig. 6 shows the pulse height distribution for one of the counters (adding the pulse heights from the two tubes). The Landau shape is clearly evident. Finally, at LAMPF, Bill Marterer has designed and is constructing the mountings for these counters. They should be in place soon after the remounting of the shield is complete and certainly by the end of the year.

\section{e) LSND Plans and LSU's Role}

LSND intends to begin taking beam in May 1993. Initial running will be with the old beamstop configuration. With just one year of good data we should be able to improve upon all the E645 results discussed at the beginning of this section. After the 1993 run we hope to carry out the beam stop upgrade discussed above. Then with an additional two years of running LSND can make a significant measurement of both $v_{\mu} \rightarrow v_{\tau}$ (by $v_{\mu}$ disappearance) and the strange contribution to the proton spin (via $v p \rightarrow v p$ ) as well as surpassing the limits shown in Figure 2 for both $\bar{v}_{\mu} \rightarrow \bar{v}_{e}$ and $v_{\mu} \rightarrow v_{e}$. If the DAQ system performs as expected we should also be able to lower the trigger threshold to a level where we can become an astrophysical neutrino observatory as well.

Building on our experience from E645 LSU will initially concentrate on the $\bar{v}_{\mu} \rightarrow \bar{v}_{e}$ decayat-rest oscillation signal. This is expected to be the thesis topic for our student, Mr. Gunasingha. Both LSU faculty members (Imlay and Metcalf) will spend significant periods of time at LAMPF (including a possible sabbatical for Prof. Imlay) helping Mr. Gunasingha to commission and maintain the veto shield as well as aiding in data taking and analysis. We also intend to carry out significant analysis work at LSU using a cluster of VAX workstations and two DECstation 5000's which belong to our group. Prof. Metcalf has already installed and tested the LSND Monte Carlo on the cluster and is preparing to use it to study backgrounds to the $\bar{v}_{\mu} \rightarrow \bar{v}_{e}$ signal. This work will receive much more attention next year with the shield recommissioned and LSND taking data. 


\section{The ZEUS Experiment at HERA}

\section{Introduction}

In 1988 the LSU HEP group decided to join the ZEUS experiment. This experiment is studying collisions of $30 \mathrm{GeV}$ electrons with $820 \mathrm{GeV}$ protons at HERA, a new storage ring at DESY. HERA is attractive because of its physics potential and because it is an unique facility. Participation in ZEUS also allows the LSU group to maintain a strong collider program in the period preceding SSC operations.

The ZEUS barrel calorimeter was built by U.S. Institutions. When we joined ZEUS a prototype module was being tested at ANL and preparations were underway to start construction of the 32 barrel modules. With HERA scheduled for completion in 1991 it was important to build the modules as rapidly as possible. We decided we could best help out by taking over the preparation of the wave length shifter bars for the electromagnetic part of the calorimeter from Ohio State University allowing OSU to concentrate on the rest of the optical components. The construction of the barrel calorimeter was completed in early 1991. The details of the BEMC waveguide construction are discussed in section 4.

The U. S. institutions on ZEUS set up a test beam at Fermilab (E790 at Lab E) to study the response of the calorimeter modules. Tests were completed in August 1991. LSU has played an important role in this test beam work which is discussed in section 5 .

ZEUS started data taking this summer. Two papers on early physics results are in preparation. Section 6 discusses ZEUS installation and early results. Section 7 discusses plans for offline analysis and computing at LSU.

\section{Physics at HERA}

HERA will permit the study of e-p collisions at four-momentum transfer squared, $Q^{2}$, up to $10^{5} \mathrm{GeV}^{2}$ at a center of mass energy $\sqrt{5}$ of $310 \mathrm{GeV}$. At HERA the neutral current $(\mathrm{ep} \rightarrow \mathrm{eX}$ ) and charged current $(e p \rightarrow v X)$ reactions will have comparable rates since $Q_{\max }^{2}>M_{w}^{2}$. Expected rates for $200 \mathrm{pb}^{-1}$ of data are shown in Fig.7. Measurement of the proton structure function in this newly opened kinematic region will be a priority for ZEUS. The large $\mathrm{Q}^{2}$ range will permit a much more stringent test of $\mathrm{QCD}$ as well as searches for quark or lepton substructure. 

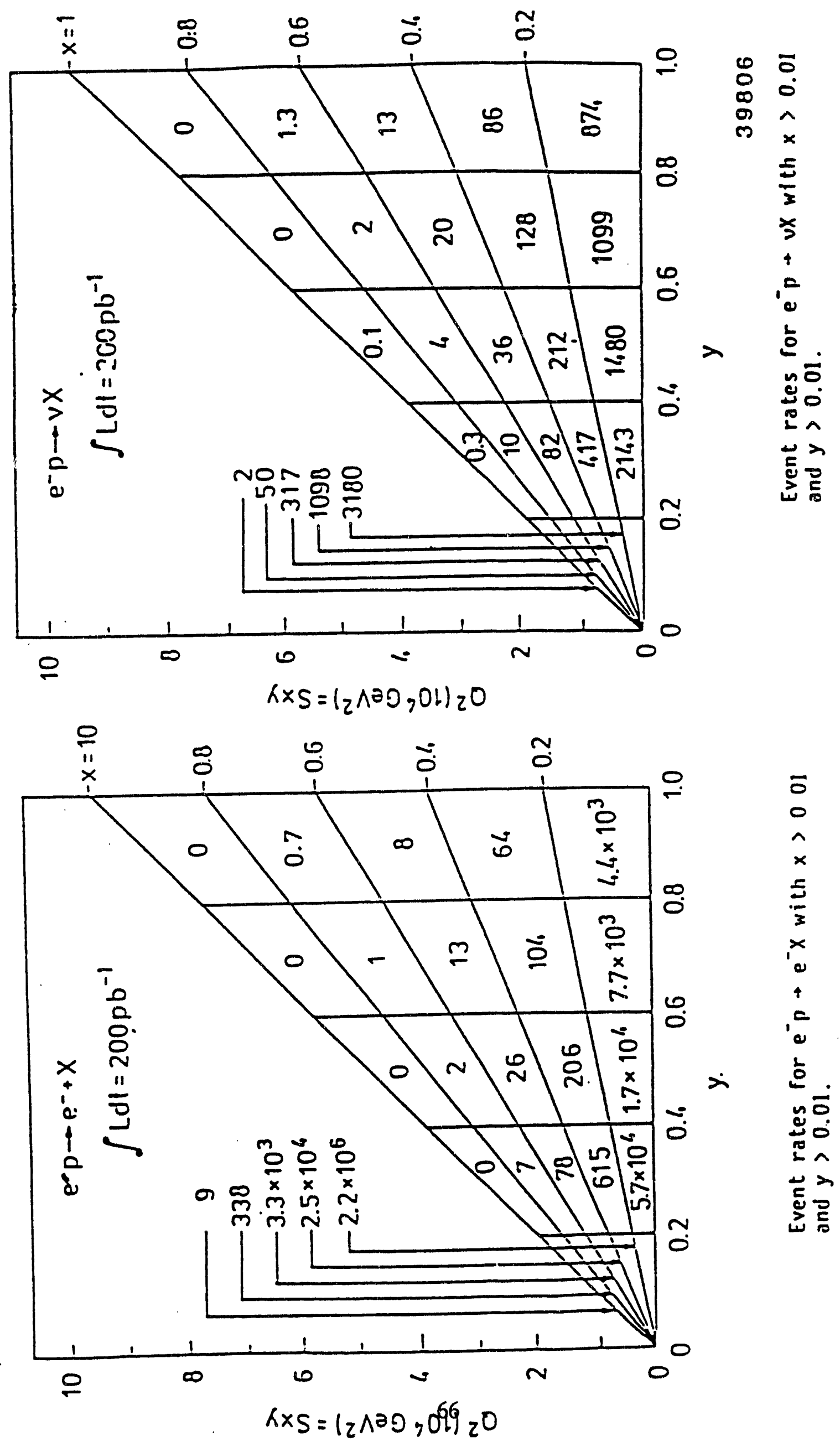
HERA will also be able to search for new particles such as excited leptons, leptoquarks, the $t$ quark, SUSY particles and new bosons $\left(Z^{\prime}, Z_{R}, W_{R}^{ \pm}\right)$. The most complete discussion is in the report "Physics at HERA" from the 1987 and the 1991 DESY workshops. ${ }^{12}$ The 1988 Snowmass Summer Study 13 reviews the physics potential of HERA and other machines for new particle searches. Overall HERA compares well. We note that studies of lepton nucleon scattering have had a major role in forming our present picture of particle physics (scaling, neutral currents, partons, QCD). We expect that HERA will have a similar impact.

\section{The ZEUS Detector}

\section{a. General Description}

The ZEUS detector is shown in Figs. $8 \mathrm{a}$ and $8 \mathrm{~b}$. Inside the coil of the $1.8 \mathrm{~T}$ solenoidal magnetic field are a vertex detector (VXD) and a central tracking detector (CTD). In the forward (proton) direction are the forward tracking chamber (FTD) and a transition radiation detector (TRD). In the rear (electron) direction is the rear tracking detector (RTD). Surrounding the coil and tracking detector are three high resolution calorimeters (FCAL, BCAL, and RCAL). All three calorimeters are similar in construction. U.S. Institutions are responsible for the Barrel Calorimeter (BCAL) which is discussed in more detail in the next section. Surrounding these calorimeters are the backing calorimeter (BAC), the forward muon spectrometer (FMU) and the barrel and rear muon detector (BMU,RMU).

The decays of short lived particles can be detected in a vertex detector which has a timeexpansion type drift cell.

The central track detector consists of a cylindrical jet type drift chamber with an outer radius of $85 \mathrm{~cm}$ and an overall length of $240 \mathrm{~cm}$. Track position and dE/dx loss are measured in 9 superlayers each with 8 layers of sense wires. Four of the superlayers have stereo wires. A resolution of $100 \mu \mathrm{m}$ is expected, leading to a momentum resolution of $\sigma(p) / p=0.002 p \oplus 0.003$ ( $p$ in $\mathrm{GeV} / \mathrm{c}$ ) for a magnetic field of $1.8 \mathrm{~T}$. Particle tracking at small forward and backward angles to the beam is aided by four planar drift chambers providing a momentum measurement with $\sigma(p) / p=0.01 \cdot p$ at a forward angle of $140 \mathrm{mrad}$.

Electron identification is performed with $\mathrm{dE} / \mathrm{dx}$ information from the tracking detectors and with the calorimeter. In the forward direction a transition radiation detector, consisting of four 


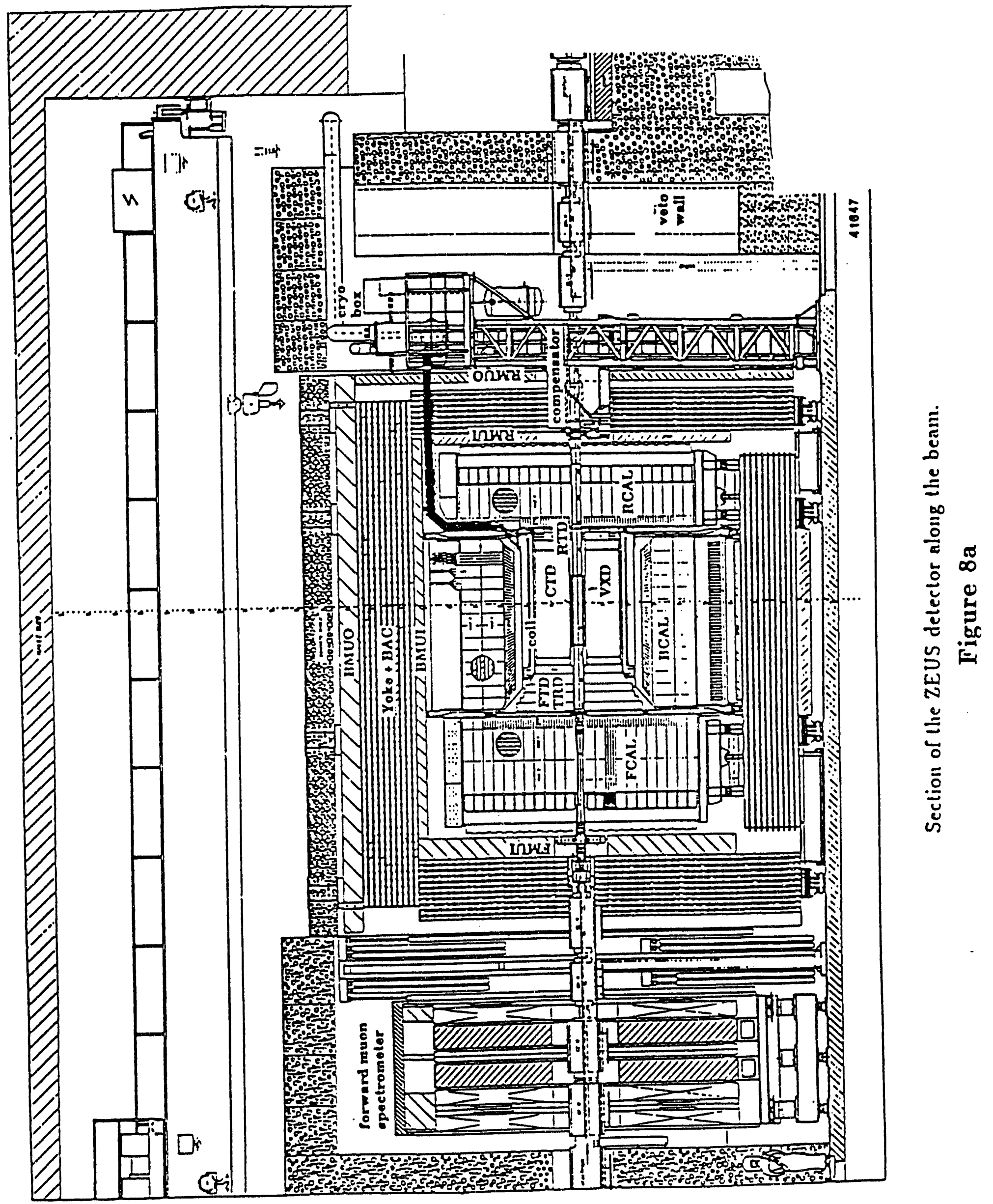




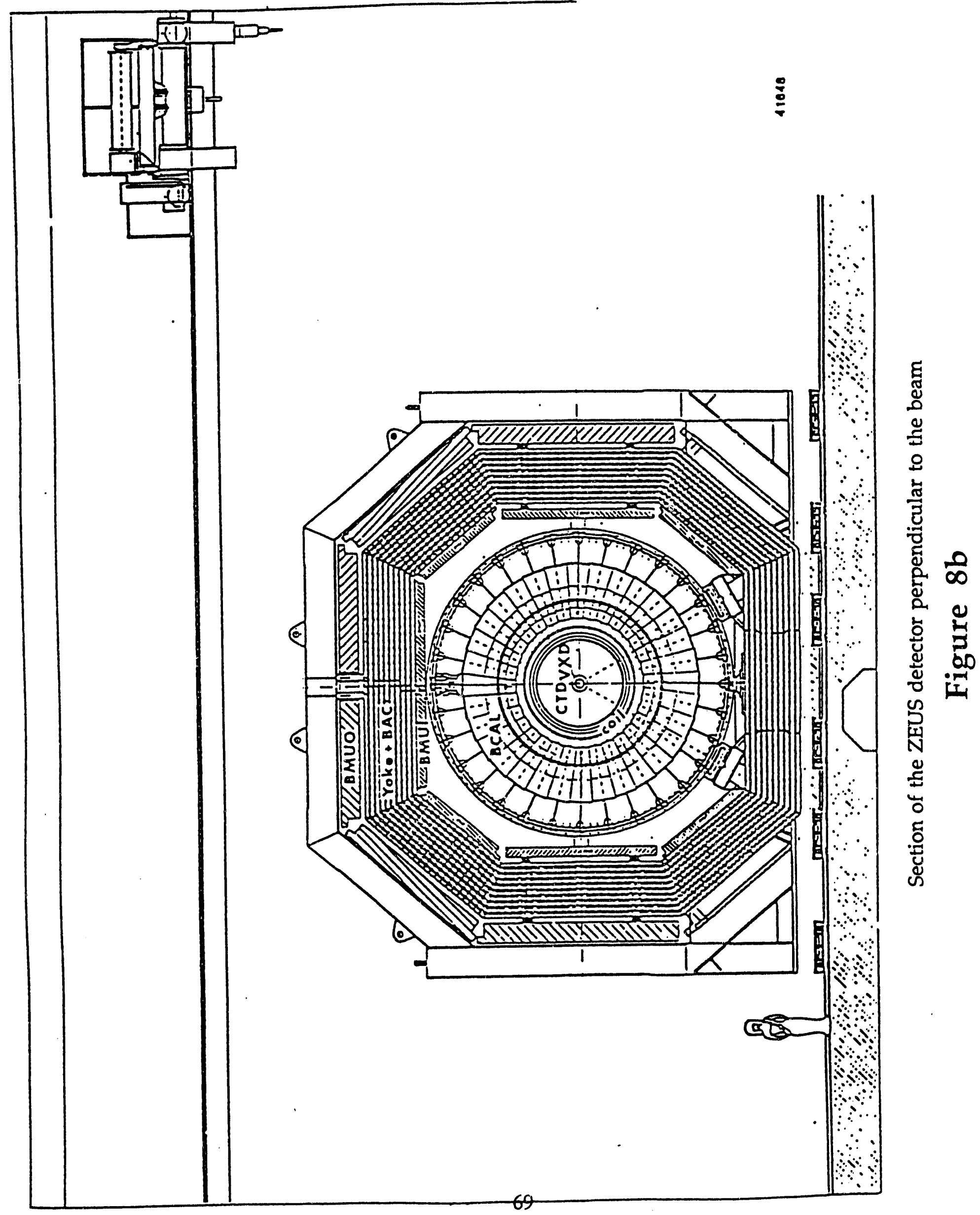


modules, yields an additional hadron rejection factor of about 100 for momenta below $30 \mathrm{GeV} / \mathrm{c}$. In order to identify electrons within dense jets, space is provided for insertion of a detector such as a silicon pad detector in the calorimeter at a depth of 3-6 radiation lengths. A hadron-electron rejection of 30-200 can be obtained with one layer. The combined hadron rejection including the information from the silicon pad detector is well above $10^{4}$.

The purpose of the backing calorimeter is to measure the energy of late showering particles. The backing calorimeter uses as absorber the iron plates which form the magnet yoke. Aluminum tubes operated in proportional mode are used for read out. The expected energy resolution for hadrons is $\sigma(E) / E=1.0 / \sqrt{E}$. ( $E$ in $\mathrm{GeV}$ ).

Muons are detected in the forward direction in a spectrometer using drift- and limited streamer tube chambers plus scintillation counters interspersed between the magnetized iron yoke and magnetized iron toroids. The momentum resolution for $100 \mathrm{GeV} / \mathrm{c}$ muons is $\sigma(\mathrm{p}) / \mathrm{p}=23 \%$. In the barrel and rear detectors muons are detected by limited streamer tube chambers before, in between and behind the backing calorimeter. The momentum resolution is $30 \%$ at $20 \mathrm{GeV} / \mathrm{c}$. The pion (kaon) rejection factors are $1000(100)$ at $40 \mathrm{GeV} / \mathrm{c}$ in the forward direction, and $700(350)$ at $10 \mathrm{GeV} / \mathrm{c}$ in the barrel region.

A leading proton spectrometer detects very forward produced protons with $\chi_{\mathrm{L}}>0.3$. The spectrometer uses proton beam line magnets and six miniature high resolution chambers installed in Roman pots very close to the beam at distances between 20 and $90 \mathrm{~m}$ from the interaction point. A momentum resolution of $\sigma(p) / p<1 \%$ is expected.

Electron and photon detectors are installed some 30 to $100 \mathrm{~m}$ downstream in the electron beam direction to measure the luminosity and tag small $\mathrm{Q}^{2}$ processes.

The magnetic solenoid is superconducting and provides a field of $1.8 \mathrm{~T}$. It has an inner radius of $86 \mathrm{~cm}$, a length of $280 \mathrm{~cm}$ and is 0.9 radiation lengths thick. A compensating solenoid is installed in the rear of the detector.

The detector is structurally subdivided into the inner components supported by the bottom yoke, and the clam shells carrying most of the backing calorimeter and the muon detectors. The clam shells can be retracted sideways to provide access to the detector. 


\section{b. The ZEUS Calorimeter}

The calorimeter consists of depleted uranium plates interleaved with plastic scintillator in order to achieve compensation and the best possible energy resolution for hadrons. ${ }^{14}$ The scintillation plates form towers which are read out via wavelength shifter bars, light guides and photomultipliers. The calorimeter is segmented longitudinally into an electromagnetic (EMC) and one or two hadronic (HAC) sections. Typical tower sizes are $5 \mathrm{~cm} \times 20 \mathrm{~cm}$ in the electromagnetic section and $20 \mathrm{~cm} \times 20 \mathrm{~cm}$ in the hadronic section. The calorimeter is divided into a forward, a barrel and a rear part with 7,5, and 4 absorption lengths, respectively. The solid angle coverage corresponds to $99.8 \%$ in the forward hemisphere and $99.5 \%$ in the backward hemisphere. The expected energy resolutions are for electrons $\sigma(E) / E=0.18 / \sqrt{E} \oplus 1 \%(\oplus$ stands for addition in quadrature) and for hadrons $\sigma(E) / E=0.35 / \sqrt{E} \oplus 2 \%(E$ in $\mathrm{GeV})$. Results from the Fermilab test beam are discussed in section 5 .

The barrel calorimeter (BCAL) consists of 32 identical modules (see Fig. 9), each covering an $11.25^{\circ}$ wedge in azimuthal angle. The inside radius for each module is $122 \mathrm{~cm}$. The polar angle coverage is from $\theta=36.7^{\circ}$ to $\theta=129.1^{\circ}$. A module is divided into three segments in depth, called the electromagnetic section (BEMC), consisting of 21 layers of depleted uranium 23 $\chi_{0}$ or $1 \lambda$ deep, and the two hadronic sections (BHAC1 and BHAC2), each 49 layers of depleted uranium, $53 \chi_{0}$ or $2 \lambda$ deep.

Within each module, the electromagnetic section is divided into projective towers with nominal dimensions of $5 \mathrm{~cm} \times 24 \mathrm{~cm}$ at the front face. In the two hadronic sections, the towers are nonprojective in $\theta$. Except at the ends four BEMC subtowers are followed by a single hadronic tower. Readout of the scintillation light from the individual towers in all three sections is accomplished using the two wave length shifter bars (WLS), placed one on each of the included faces of the modules and connected through light guides to photomultiplier tubes. Each module is closed by aluminum side panels that are used to retain the WLS plates. Each aluminum side panel has a $1 \mathrm{~mm}$ lead sheet attached to it to reduce the effects of photons showering in the WLS. The BEMC is projective both in $\theta$ and $\phi$, while the $B H A C$ is only projective in $\phi$, resulting in rectangular WLS-tiles.

\section{Construction of the BEMC Waveshifters}

As discussed in the introduction to the ZEUS section LSU produced the wavelength shifter bars for the electromagnetic part of the barrel calorimeter (BEMC). 


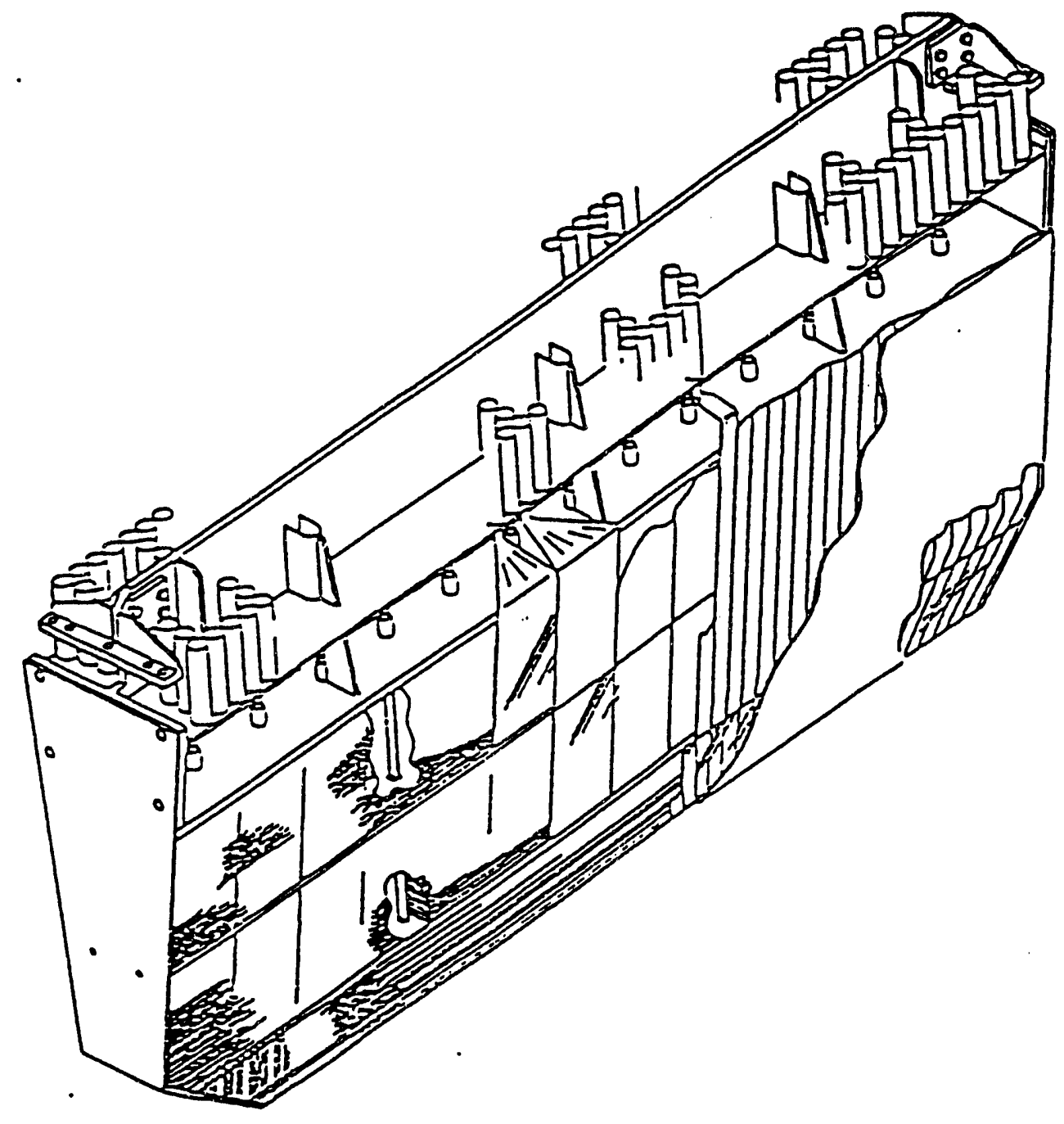

Isometric view of $\mathrm{BCAL}$ module

Figure 9 
We hired three technicians in 1989 to set up the production facility at LSU for BEMC waveshifter bars. A duplicate scanning table was prepared at OSU and sent to LSU. A commercial oven for annealing pipes at LSU was bought. Our technicians made a number of trips to OSU that summer to both learn the procedures and to help in the preparation of waveshifters for the first module at OSU. Our technicians also went to ANL to install the EMC waveshifters on several modules.

The procedure for preparation of the WLS is now briefly described:

The Y-7 WLS sheets were laser cut by Laser Services Inc. into EMC optical components. They were bent at LSU to conform to the geometry of the barrel calorimeter. Ninety degree bends in the pipes were formed using infrared lamps and special jigs which make no contact with the hot plastic. Fingers leading to the transition piece were hand formed after heating. After bending, the finger ends were milled to join a transition piece leading to the photomultiplier tube. The light pipes was then glued to the transition piece. To avoid crazing, the WLS were annealed in an oven for 8-10 hours before gluing. Finally each WLS was washed in an ultrasound bath of mild soap and distilled water. The bottom of each WLS piece was painted with Bicron reflecting paint to decrease longitudinal non-uniformities, and the long straight section of the pipe which transports the light to the photomultipliers was wrapped with $100 \mu \mathrm{m}$ aluminized mylar.

The scintillator tiles and waveshifters have light-yield non-uniformities caused by thickness variations $( \pm 5 \%)$ in the plates. The light-yield also depends on the distance the scintillator tiles are from the phototubes. We correct for light-yield non-uniformity by application of a reflective mask onto the waveshifter. LSU prepared these reflection masks. The light collection uniformity of each piece of scintillator and coupled waveshifter was measured using $x-y$ scanning tables. The LSU scanning table is computer controlled via a CAMAC based data acquisition system modeled after the Ohio State University system, but utilizing our Vaxstation 3200 computer. The scan results prove to be reproducible point by point to slightly better than $1 \%$.

The results of the scans for the WLS and scintillator tiles were used to prepare the reflection masks. First, a pattern (negative image) of black rectangles whose density reflects the light-yield results from the scan was printed on a transparency. The transparency was then used to expose photo-sensitive aluminum plates which are attached to the face of the WLS opposite the scintillator tiles. The reflection masks have been shown to remove the non-uniformities leaving a residual non-uniformity of less than $1 \%$. As a result of the ZEUS production effort, LSU now has a 
photographic exposure facility to make reflection masks.

LSU was able to begin production and scanning of EMC waveguides in Baton Rouge in September 1989. By the end of the year we had achieved our goal of producing 2 modules of WLS pipes per month. Construction was finished in March of 1991.

\section{Fermilab Testing of ZEUS Modules}

During assembly at Argonne each module was tested in a cosmic ray stand as well as with radioactive sources. These tests helped insure that there were no errors in the production of any module. They were not sufficient, however, to study the performance of the detector in detail.

Therefore, the U.S. ZEUS collaborators established a test beam in the neutrino area at Fermilab to study in detail the response of a few modules. LSU played a key role in this test beam effort. Data taking ended by August 1991. An LSU postdoc, Hong Joo Kim, spent the entire period from February to September 1990 and 3 months the next summer at Fermilab. An LSU graduate student, Lufan Chen, spent two months there both summers. Our newest postdoc, Subramanian Kartik, was present for the last month of the testbeam run. All three LSU faculty members also made several trips to Fermilab to help on the test beam work.

The test beam delivers a mixture of electrons, muons and hadrons (principally pions and protons) with an intensity of up to $2 \times 10^{11}$ particles/spill and with momenta tunable from 30 to $150 \mathrm{GeV} / \mathrm{c}$. Besides his duties as supervisor of the test beam running, Hong Joo Kim was the member of E790 who learned how to tune the beam and adjust its momentum using Fermilab's EPICURE system. He was always on duty during scans involving changes in beam momentum.

The test beam facility consisted of a set of scintillators for triggering; three dipole magnets for momentum determination; a set of $8 \mathrm{drift}$ chambers (each with $\mathrm{x}$ and $\mathrm{y}$ planes) for momentum and position measurement; stands for holding modules to be tested and a variety of particle identification devices. An array of TRD's upstream of the dipoles was used to identify electrons; a gas Cherenkov counter just downstream from the dipoles was used to tag protons and an ironliquid scintillator sandwich (actually a section from the E770 neutrino detector) was used as a muon filter and a backing calorimeter to measure the energy leakage from modules in hadronic events. A stand in front of this backing calorimeter held up to 4 modules. It rotated horizontally and vertically so that beam particles hit the central modules on the stand in the same way as particles of a fixed $\theta$ and $\phi$ coming from the interaction region at HERA. This was especially 
important in studying the response of the projective EMC towers at the front of the module. Behind the backing calorimeter was another stand which held a single module. It was adjustable in the horizontal and vertical directions perpendicular to the axis of the module to study the response of the non-projective HAC towers to muons. Two of the scintillators and two of the drift chambers were also behind the muon filter to establish a muon trigger and position.

As a natural extension of his responsibility for the beam, Hong Joo Kim (along with a postdoc from Columbia) was responsible for installing, commissioning and operating all of the drift chambers and related electronics. He also wrote the software for tracking and momentum determination.

As an indication of BCAL module performance, Figure 10 shows the energy deposited in the electromagnetic calorimeter (EMC) divided by the total energy deposited in the EMC plus the hadronic calorimeter ( $\mathrm{HACl}$ and $\mathrm{HAC} 2)$ for various types of beam particles. Because electromagnetic showers are largely contained in the EMC this ratio should be approximately 1 for electrons. Ii will be near zero for particles which shower in the HAC and some intermediate value for particles which don't shower or for hadronic showers that begin in the EMC.

Analysis of the ZEUS beam test data is nearly complete. Results were presented at the recent XXVI International Conference on High Energy Physics in Dallas by Hong Joo Kim of LSU. The energy resolution was measured to be $35 \% / \sqrt{E}$ for hadrons and $19 \% / \sqrt{E}$ for electrons, close to design goals. The tower to tower and module to module uniformities were better than $1 \%$, the energy nonlinearity less than $1 \%$ and the response for electrons and hadrons nearly equal. Kim's talk is given in Appendix A.

The LSU group studied the response of the hadronic modules to muons. This work was largely competed by early 1992. Results are presented in ZEUS Note 92-023, which is included as Appendix B in this proposal. Kim, Kartik and Chen all were heavily involved in the analysis assisted by LSU faculty members (especially W. Metcalf). McNeil provided the muon simulation for comparison with the data.

\section{ZEUS installation and early operations}

The 32 BCAL modules were completed on schedule in March 1991. They were installed in the ZEUS detector by June, except for the 3 modules being tested at Fermilab. These final 3 modules were installed in the fall of 1991. The LSU technician, S Khosravi, spent several months 

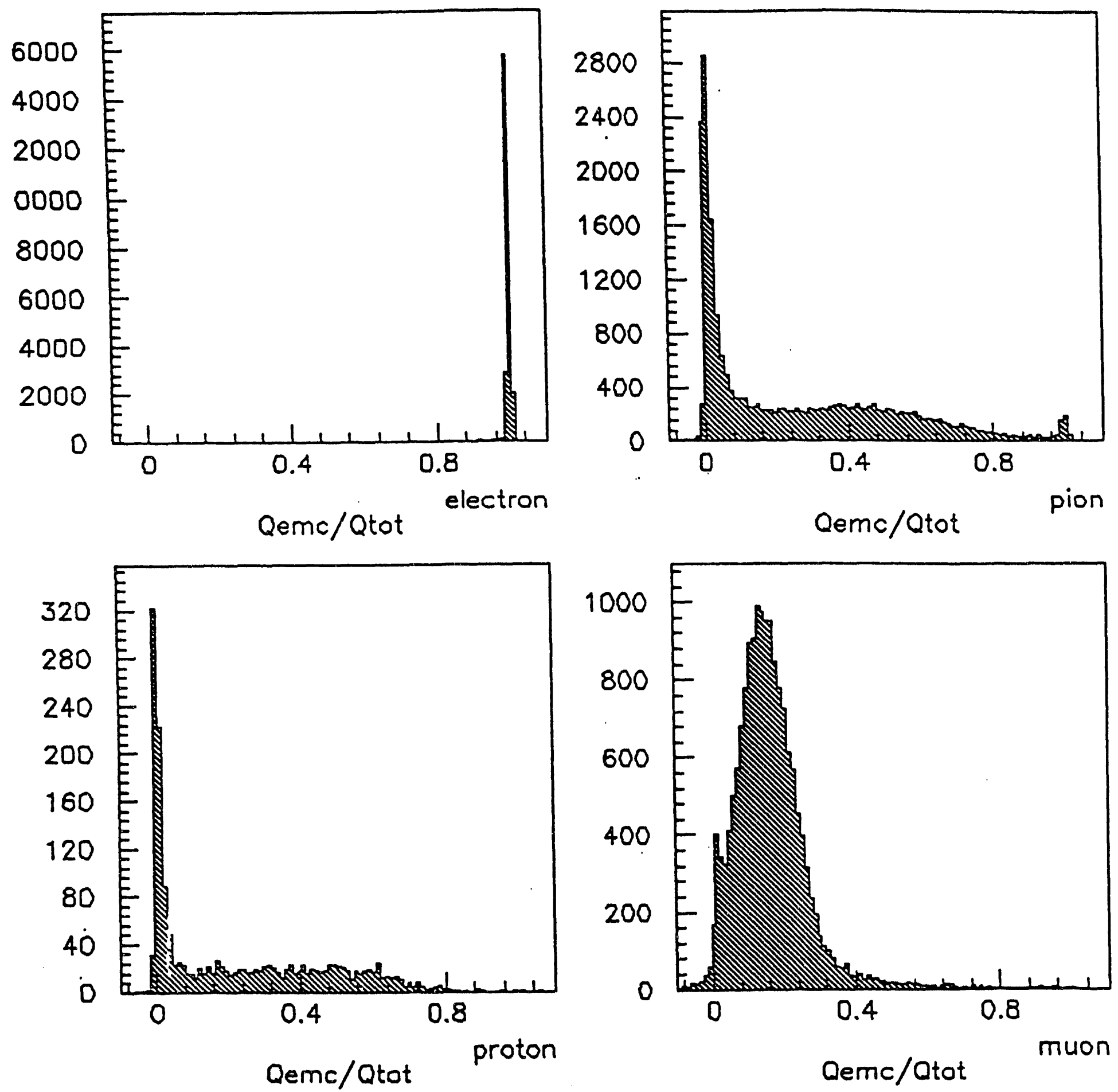

Response of BCAL

Figure 10 
at DESY in 1991 helping test and install the BCAL modules.

The HERA accelerator was completed and began commissioning in the fall of 1991. Electron and proton bunches collided for the first time on October, 19,1991. In late March of 1992 ZEUS rolled into position. By earlier June some luminosity was observed. Substantial beam gas data was also taken with the proton beam for testing and debugging ZEUS. By August ZEUS had $2.5 \mathrm{nb}^{-1}$ of integrated luminosity.

Kim moved to Hamburg in September 1991. He has assisted in the commissioning of the ZEUS Barrel Calorimeter and preparation for analysis of data. Kartik went to Hamburg in April 1992 after completion of analysis of the test beam data. Unfortunately the graduate student, Lufan Chen, who was scheduled to go to Germany in early May decided to leave the high energy group at that time. Both Professors McNeil and Imlay spent substational time at DESY this summer.

At DESY Kartik has prepared a database program for the initial ZEUS data. He is also taking the responsibility for keeping in operation some of the U.S. computer facilities at ZEUS. McNeil worked at improving the calorimeter timing using muons. Timing has proved crucial at ZEUS for rejection of background events. Imlay helped look at the effect of the B field on the calorimeter PMT's.

It is our plan to maintain from the onset of HERA data taking two physicists at DESY. Initially this consists of the two postdoctoral researchers. Eventually we would like to have graduate students and postdoctorals cycle between LSU and DESY and maintain strong analysis efforts at both places. The LSU faculty intends to travel frequently to DESY consistent with our teachings.

B. Lohr of DESY presented first results from ZEUS at the Dallas International High Energy Conference in August. Fig. 11 compares the preliminary photoproduction cross section at $200 \mathrm{GeV}$ of $153 \pm 16 \pm 35 \mu \mathrm{b}$ with previous data and models. The deep inelastic neutral current sample of 360 events is shown in Fig. 12. Leptoquark limits were also presented. Two papers on this early data are in preparation. At low luminosity we will concentrate on the search for events from $\mathrm{e}^{*} \rightarrow \mathrm{e}+\gamma$. The signature is very clean. Fig. 13 compares the estimated sensitivity for $1 \mathrm{pb}^{-}$ 1 with LEP limits.

The LSU group has been participating in the exotic physics group which is searching for leptoquarks, excited leptons and other exotic particles. The LSU group is presently concentrating 
on the excited lepton search. Hong Joo Kim has played the major role in organizing the ZEUS excited lepton effort. 
Photoproduction
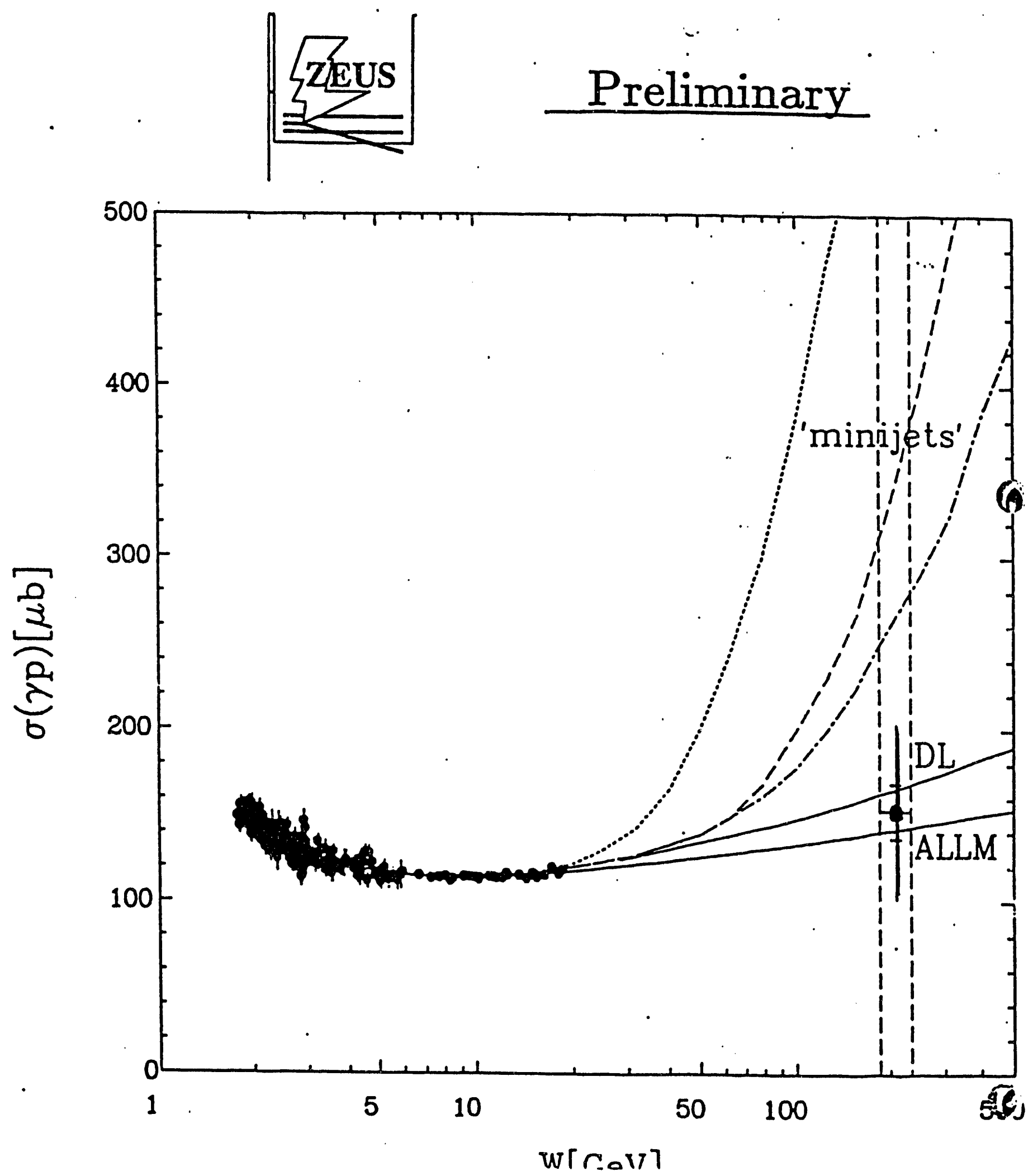

Figure 11 
preliminary

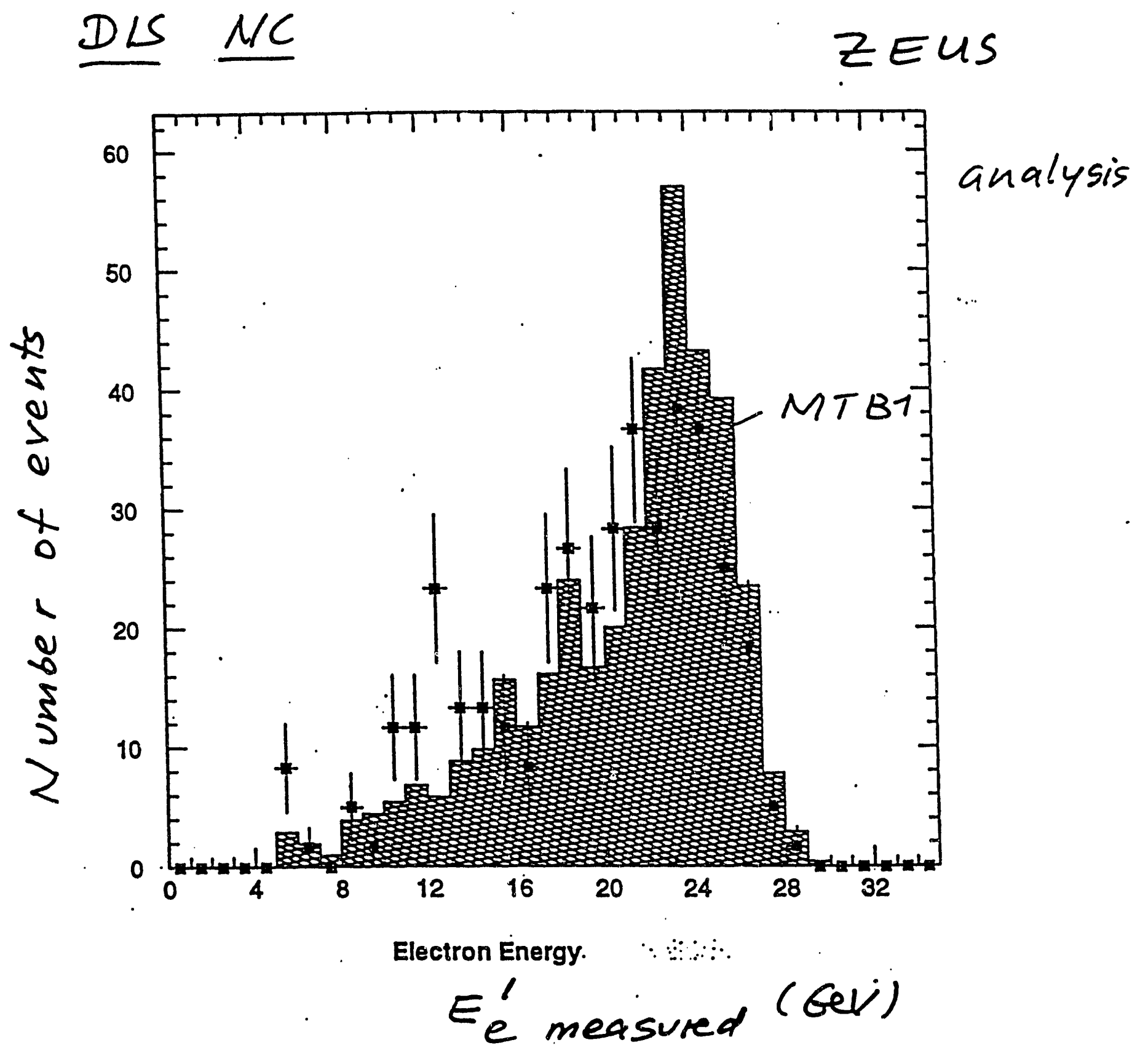

$\underset{80}{\text { Figure }}{ }^{12 a}$ 
NC
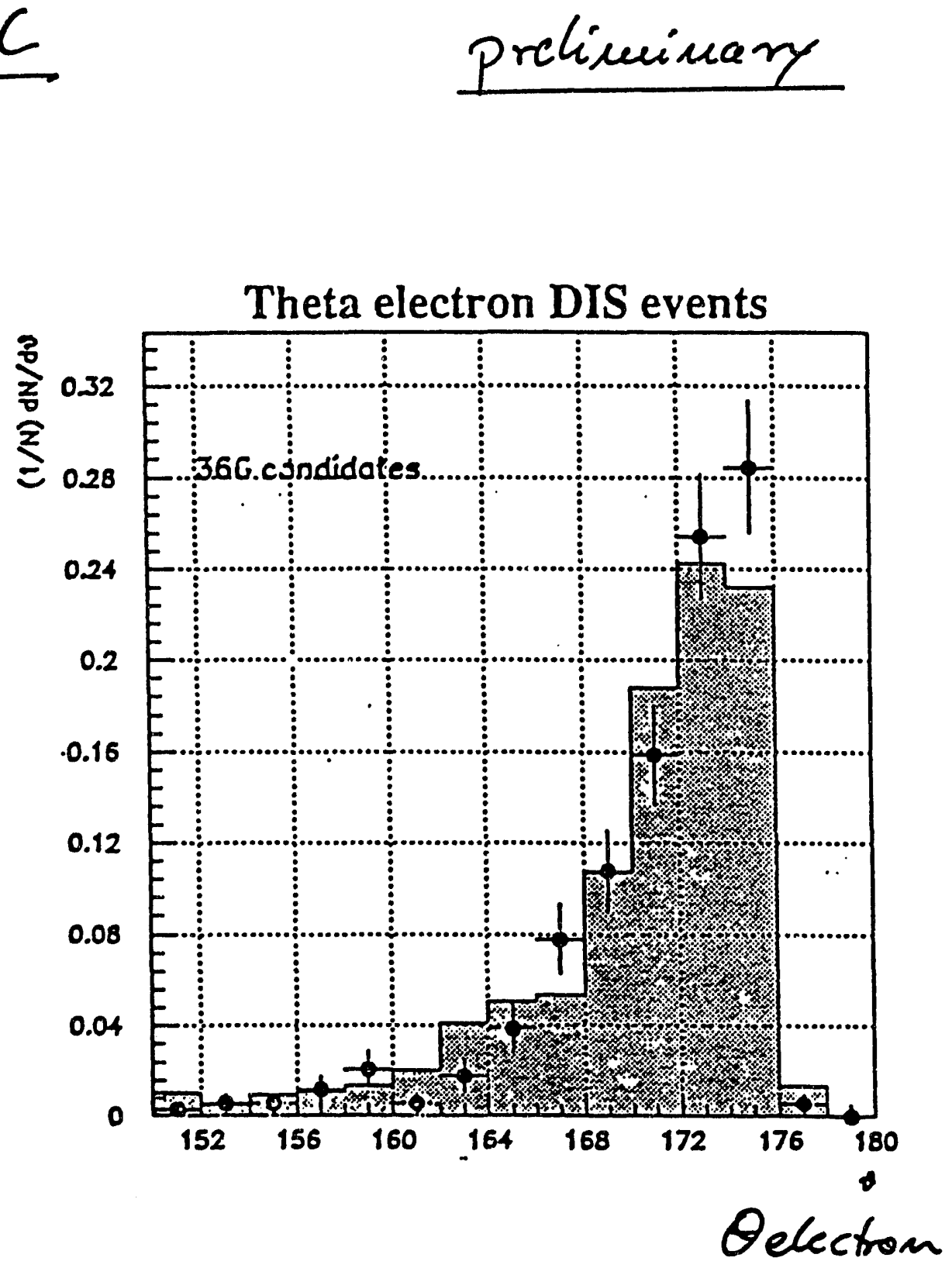

Figure 12b 


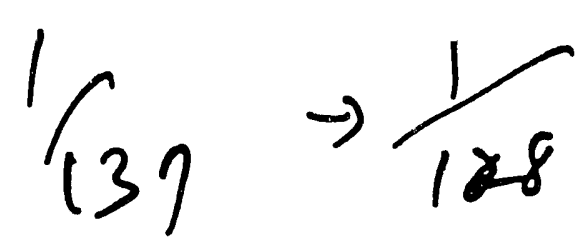

$\mathrm{E}^{*}$ limit in ZEUS with 1 pb-1

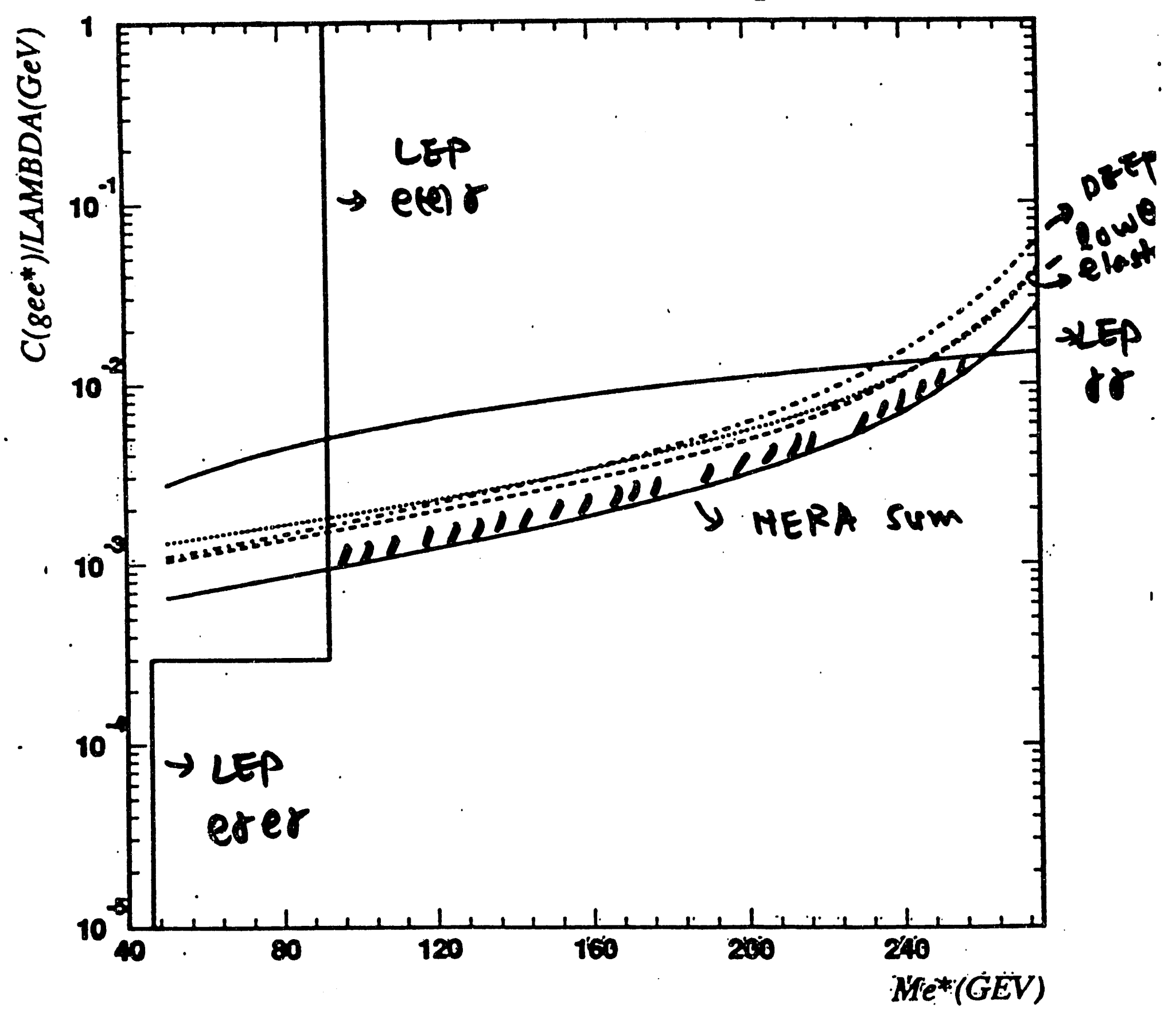

Figure 13 


\section{ZEUS Offline Analysis/Computing at LSU}

\section{a. Introduction}

During the summer of $1991 \mathrm{McNeil}$ visited DESY and obtained copies of the current ZEUS offline analysis software. This software was transported to LSU where it is now being installed on both Vaxstations (VMS version) and Decstations (UNIX version). The offline software for ZEUS consists of the following program packages:

- ZDIS - ZEEUS Interface to Deep Inelastic Scattering. The Monte Carlo Generator for ZEUS.

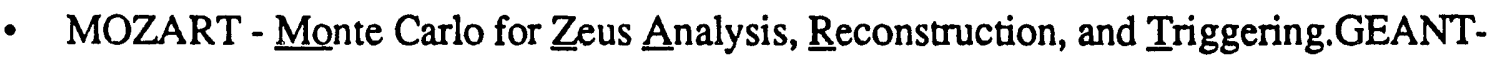
based Monte Carlo simulation for ZEUS.

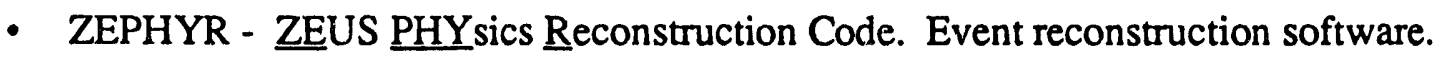

- EAZE - Effortless Analysis of ZEUS Events. Analysis software.

- LAZE - Logical Access to ZEUS Events. Display software.

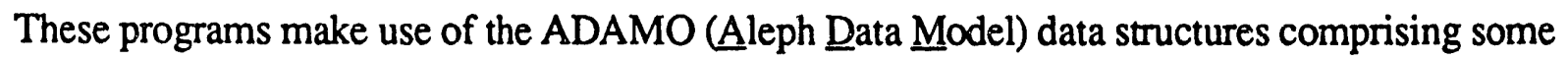
360 tables. So far we have successfully run the ZEUS software on VAXes (VMS) at LSU. One of our goals is to provide ZEUS with a facility for Monte Carlo event production.. An experiment such as ZEUS requires extensive simulation studies to determine detector performance, acceptances and to understand the systematic errors in the measurement performed at HERA. When ZEUS began taking data this year it became immediately clear that the DESY computer resources became saturated handling the data filtering, reconstruction, and analysis. We can continuously produce Monte Carlo events as a "background" process at LSU provided we have the $\mathrm{CPU} / \mathrm{memory}$, and the manpower and expertise to monitor and compile the events (from different processors). LSU has considerable computing resources which could be utilized to provide ZEUS collaborators with Monte Carlo events via 8mm tape. 


\section{b: Computing resources for ZEUS analysis at LSU}

At LSU we have four VAXstations purchased with DOE funds. These workstations are on a VAX cluster of which the Experimental High Energy, Intermediate Energy/Nuclear, Cosmic Ray, and Neutrino Astrophysics groups are members. A total of 9 VAXstations, VAX 3500, and VAX 4000 are on the cluster and the computing resources are shared. With additional memory and system resources these computers can be used for ZEUS analysis and simulation. Funds are being requested to allow the large ZEUS programs to run on these workstations.

During 1992 we purchased three Decstation 5000's on DOE funds to add to our one existing Decstation 5000 purchased for the HEP Group by the LSU Physics Department. Two of these workstations were sent to DESY to be used by our on-site postdocs and graduate students. We also purchased additional memory for all the workstations to allow them to handle the large ZEUS programs and 3GB of external SCSI Disks (one of which was sent to DESY) to store the ZEUS software and allow temporary data storage. The two Decstations remaining will be used primarily to run ZEUS analysis programs and Monte Carlo event simulations for the ZEUS experiment.

Additional free CPU is present at LSU and we are nuaking arrangements to take advantage of it. SNCC (System Network Computing Center) owns a VAX 8800 and manages a cluster of about 20 Vaxstation 3100's (owned by the Engineering and Computer Science Departments). An ethernet connection exists between our computers and those at SNCC and elsewhere at LSU. The Vax 8800 has sufficient memory to run these programs and the high energy group maintains an RA81 disk on this machine. However, the $8800 \mathrm{CPU}$ is utilized throughout the university for research, education, and software development. A special queue has been arranged whereby one of our batch jobs can run on these Vaxstations as long as no other interactive or batch users are present. We have found at present these machines are available in this fashion at least $50 \%$ of the time. To make the most use of these substantial computing resources we request additional memory for installation on 10 of the vaxstations and one additional vaxstations to be used to coordinate the simulating and become a central node for collecting the simulation output.

\section{c. Manpower for ZEUS analysis at LSU}

We have hired two student workers to perform in the programming tasks and ZEUS software installation and maintenance. One student is an undergraduate physics major who obtained a fellowship from the University to work with our group to maintain the DECstations at 
LSU. We have taken on two new graduate students on the ZEUS experiment. Both students have additional coursework before going to DESY to participate in ZEUS. We have split a Research Assistantship stipend between them. They will gain experience of the ZEUS software and analysis is under the guidance of the LSU faculty. We expect one of the students would be ready to go to DESY by Summer 1993. One of our postdoctoral researchers is expected to return to LSU at the end of 1992 and will direct the analysis effort at LSU. We feel that these people together with the three LSU faculty is sufficient manpower to provide ZEUS with a dependable source of Monte Carlo events for analysis of data. 


\section{Non-Accelerator Physics}

Currently, there are two non-accelerator physics experiments in which LSU is a participant, the IMB Proton Decay Experiment and the Deep Underwater Muon And Neutrino Detector (DUMAND). Personnel involved are R.Svoboda (IMB and DUMAND), M.Cherry (IMB), R.Clark (DUMAND), and N.Beach (IMB). The latter two people are a graduate and undergraduate student, respectively. The status of both experiments is discussed below, with emphasis on tasks being done at LSU.

\section{IMB}

The IMB-3 detector shut down in March, 1991 after a serious leak. Since then we have been working on completing the data analysis and publishing papers, along with disassembling the detector and laboratory in Cleveland. In addition, preparations are underway for beam test calibration run at the KEK accelerator facility in Japan.

\section{a. IMB Data Analysis}

Data analysis continues with IMB data. In most areas, complete data sets now exist, though in some cases events are still being extracted from raw data tapes. Table 1 gives a brief summary of the status of the various IMB data sets. As can be seen, the major portions have been completed as far as producing files of agreed-upon events.

At LSU, we are now currently working on two data reduction projects. The first of these is the assembly of the final low-energy contained event sample $(<70 \mathrm{hit}$ PMT's). This involves scanning candidate events from Irvine and Boston University to decide their final disposition and "official" event interpretation. As of September, data up to October 1990 have been completed. It is estimated that this work will be done before the end of October, 1992. These data can be used to look for low light level proton decay (such as $p \rightarrow \nu K^{0}[15]$ ), and also to investigate the possible origin of gamma ray bursts (see Appendix $C$ ). Most of these events are presumably background, are are composed of a mixture of low energy neutrino interactions and nuclear spallation events caused by the passage of high enc.gy cosmic ray muons through the detector. Many of the spallation events can be rejected by their time correlation with such muons. Figure 1 shows the correlation of the time from the last muon with energy for the 1986-89 data set. Spallation events appear as the cluster in the lower left.

The second project is the extraction from tape of upward-going muons from very high energy ( $>100 \mathrm{TeV}$ ) neutrino interactions. These events were not analyzed for in previous runs due to the difficulty in separating high energy (and therefore highly ionizing) upward-going muon tracks from certain chance orientations of downward- 
Table 1: IMB Data Analysis Summary

\begin{tabular}{||l|l|l||}
\hline \hline Data Set & Scientific Goals & Status \\
\hline $\begin{array}{l}\text { contained events with } \\
>70 \text { PMT hits }\end{array}$ & $\begin{array}{l}\text { high light level proton decay } \\
\text { atmospheric neutrino flavor content }\end{array}$ & DST now completed \\
\hline $\begin{array}{l}\text { contained events with } \\
<70 \text { PMT hits }\end{array}$ & $\begin{array}{l}\text { low light level proton decay } \\
\text { supernovae and related topics }\end{array}$ & $\begin{array}{l}1986-89 \text { DST completed } \\
\text { almost done with 1990,91 }\end{array}$ \\
\hline $\begin{array}{l}\text { upward-going muons } \\
\text { with < 1800 PMT hits }\end{array}$ & $\begin{array}{l}\text { neutrino oscillations } \\
\text { neutrino astronomy }\end{array}$ & DST completed \\
\hline $\begin{array}{l}\text { upward-going muons } \\
\text { with > 1800 PMT hits }\end{array}$ & neutrinos from AGN's & $\begin{array}{l}\text { extracting events } \\
\text { from tape }\end{array}$ \\
\hline
\end{tabular}

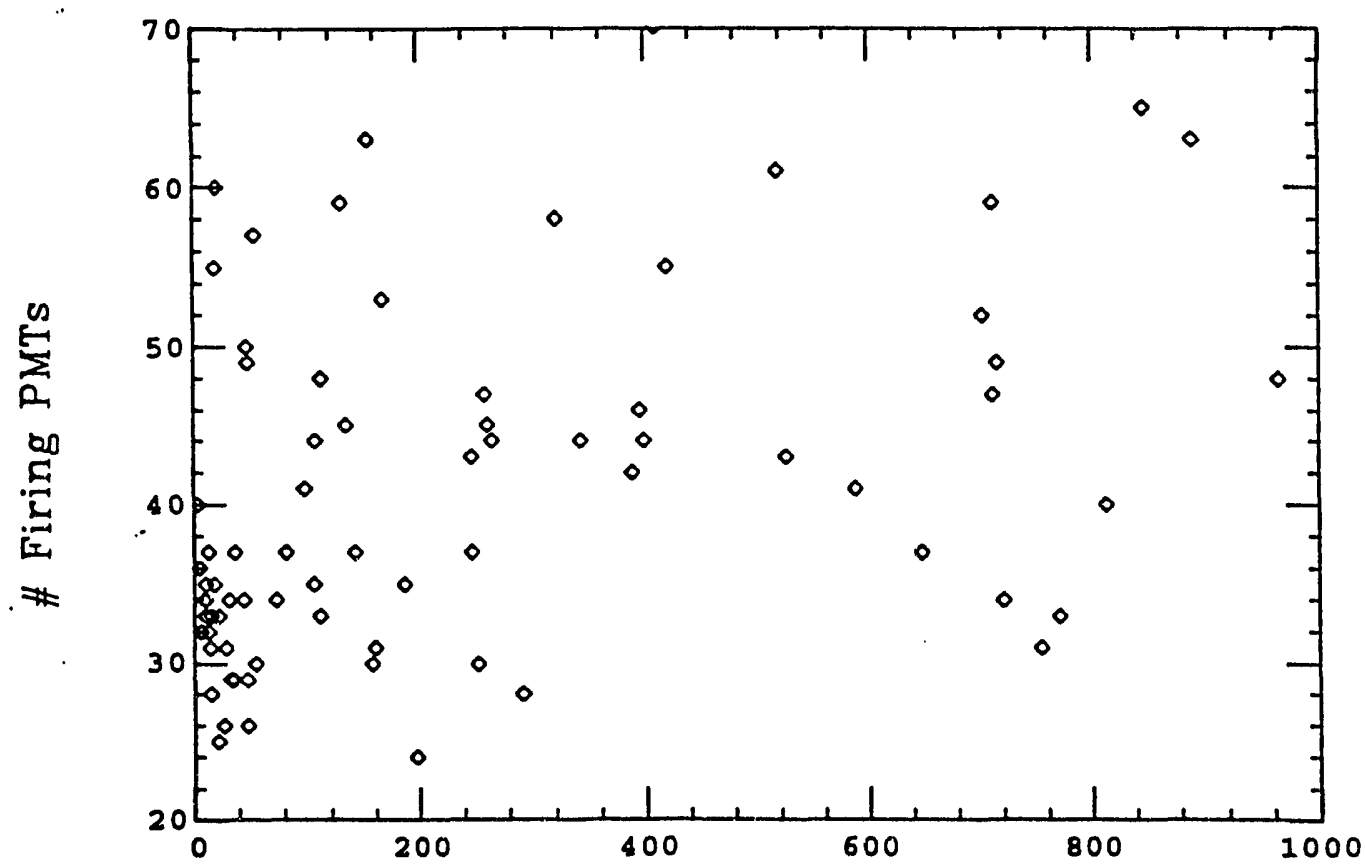

Time Since Last Muon Event (ms)

Figure 1: Time from last muon in ms versus number of PMT hits for the low energy contained data set, 1986-89. The events from nuclear spallation can clearly be seen in the lower left-hand corner. 
going multiple muon events. A cut was made at 1800 PMT's to reject all events with high light deposition. Subsequently, such interactions (the ones that were cut) were predicted to be detectable in IMB from a model of Active Galactic Nuclei (AGN) by Stecker, Done, Solomon, and Sommers [16]; and by Szabo and Protheroe [17]. Thus, the data reduction software was altered to be able to extract these events from tape, and we are now rerunning all the IMB-3 data tapes. So far, 526 live days of data have been run with the new software. This is $61 \%$ of the available IMB-3 data. About $75 \%$ of these data have been scanned. Thus, the tape extraction process should be completed by next March.

These data reduction projects require the services of Nicole Beach, a physics undergraduate who is a very experienced scanner of IMB events. This is her fourth year she has been working for the group. Funds to keep her on as a scanner until August, 1993 are requested in the budget. It is assumed that all scanning will be completed by this time.

\section{b. IMB New Results}

Recently, two papers were published on the subject of neutrino oscillations using measurements made of the background neutrino flux $[18,19]$. The first of these papers (accepted by Physical Review D, but not yet in print) is a follow-up paper to our 1991 paper [20] dealing with the relative $\nu_{\mu}$ to $\nu_{e}$ flavor content of cosmic ray neutrinos produced in the atmosphere. Though a copy of this paper is included with this proposal (Appendix C), it can be summarized by figure 2 and figure 3. These figures show the momentum distribution for single-prong contained interactions for both showering and non-showering events, respectively. The upper set of data points are for all single-prong events of the appropriate type (showering or non-showering), and the lower set of points shows the same data but with a cut to reject those events which do not show a muon decay on the long time scale TDC (out to about 7.5 $\mu \mathrm{sec}$ ). The solid and dashed lines show the predicted distribution using the flux of Lee and Koh [21]. These figures confirm our previous results and those of Kamiokande [22] that there is a serious discrepancy between the predicted and measured ratio of muon-like events to total events. For IMB, this measured ratio is $0.36 \pm 0.02$ (stat.) \pm 0.02 (syst.) in the momentum range $100<p_{e}<1500 \mathrm{MeV}$ and $300<p_{\mu}<1500 \mathrm{MeV}$. This is to be compared with the predicted ratio of $0.51 \pm 0.01$ (stat.) \pm 0.05 (syst.). Thus the discrepancy amounts to a $3 \sigma$ effect. This becomes more interesting with combined with similar results from Kamiokande. If the Soudan II detector also confirms these measurements, then the effect can probably be considered well-established. The question is, does this effect come from neutrino oscillations?

In an attempt to answer the latter question, the 3.6 year sample of upward-going 


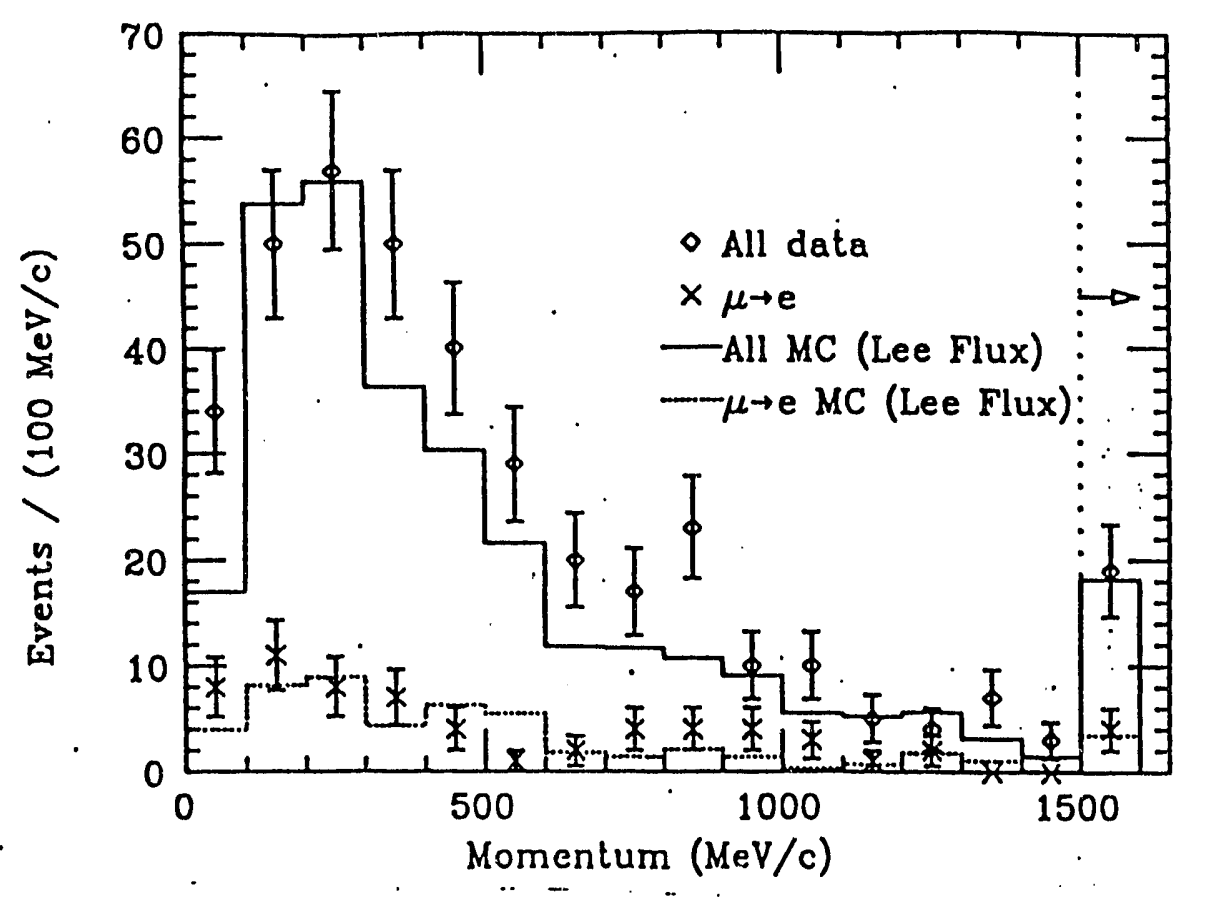

Figure 2: The momentum distribution of single-prong showering (electron-like) events. The solid line is the prediction while the upper set of points represent the data. The subset of these events with muon decay signatures is also shown (dashed line and lower points).

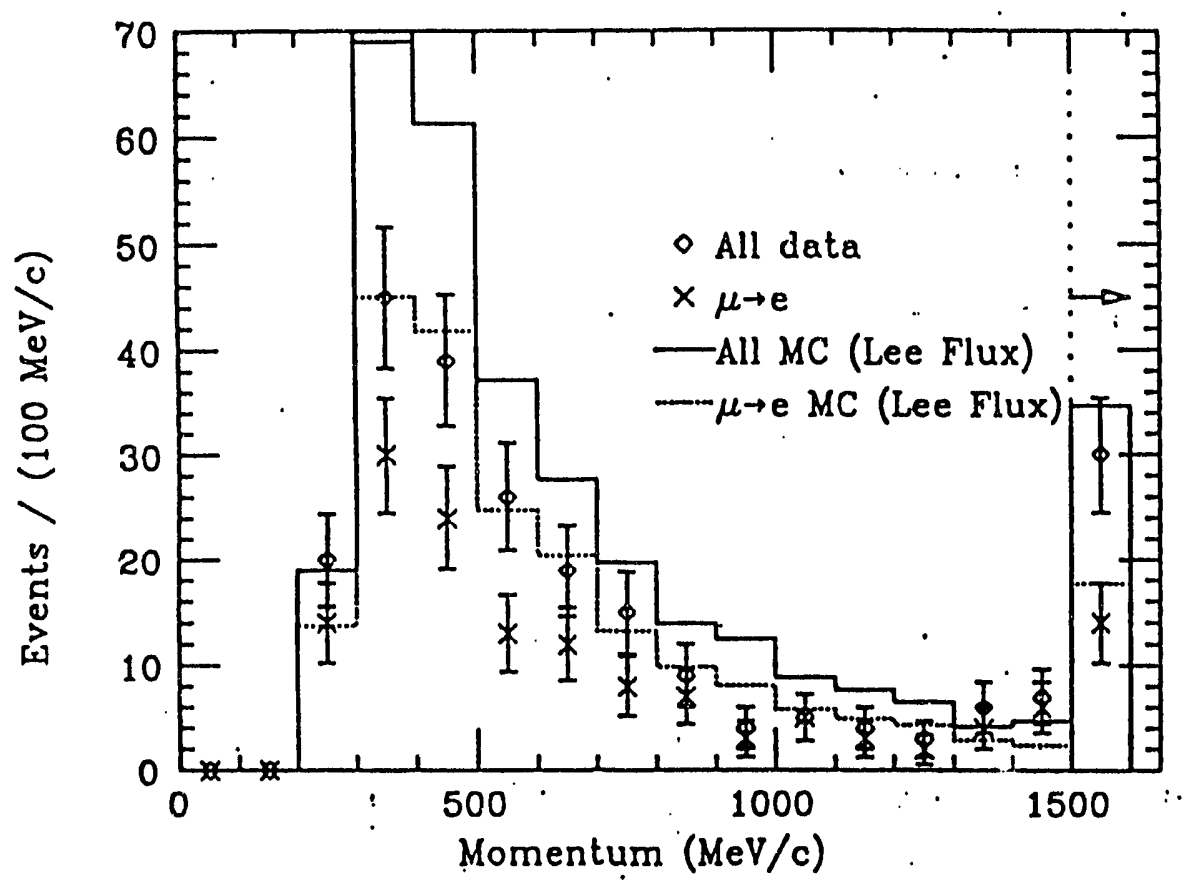

Figure 3: The momentum distribution of single-prong non-showering (muon-like) events. The solid line is the prediction while the upper set of points represent the data. The subset of these events with muon decay signatures is also shown (dashed line and lower points). 
muons was used to look for $\nu_{\mu} \rightarrow \nu_{\tau}$ oscillations in our 1992 Phys. Rev. Lett. article. The bulk of the work for this paper was done at LSU, where much of the data was sifted from tape and scanned. Talks were given by Svoboda at the FermiLab Long-Baseline Neutrino Oscillation Workshop and at the Cosmic Ray Conference in Dublin, and a poster session was presented at Neutrino '92. A copy of this paper is included with this report (Appendix C), but the results can be summarized in figure 4. The region marked "Kamioka Allowed" is the region of $\delta m^{2} \sin ^{2} 2 \theta$ space for $\nu_{\mu} \rightarrow \nu_{\tau}$ oscillations that would be allowed by the Kamiokande contained event result (the region would be quite similar for IMB). The dashed curves show exclusion regions from other experiments. The "disappearance" of $\nu_{\mu}$ due to oscillation into $\nu_{\tau}$ would manifest itself in two ways in the upward-going muon sample: (1) there would be an overall deficit of upward-going events, and (2) the spectrum would become harder, due to preferential oscillation of the low-energy $\nu_{\mu}$. Curves (A) and (B) represent exclusion regions based on these two effects. In Curve (A) the total number of events recorded $(617 \pm 25)$ is compared to the total number predicted from various combinations of cross section, parton distribution, and energy loss models. The predicted number is $600 \pm 120$ (syst.) events. Thus there is no confirmation of the $\nu_{\mu} \rightarrow \nu_{\tau}$ hypothesis from the earlier contained event analysis. Curve (A) represents the exclusion region based on non-observation of oscillations.

A a measure of spectral hardness, the ratio of stopping to through-going muons is used. Stopping upward-going muons are more likely to come from low-energy (10 $\mathrm{GeV}$ or so) neutrino interactions than the through-going ones (which have a median energy around $100 \mathrm{GeV}$ ). Because we are comparing a measured to a predicted ratio, much of the absolute error in cross-section and flux cancel out. Certainly the error is much less than the $13 \%$ statistical error in the measured ratio. The predicted ratio of stopping to through-going muons is 0.163 compared to the measured ratio of $0.160 \pm 0.019$ (stat.). Again, the $\nu_{\mu} \rightarrow \nu_{\tau}$ hypothesis is not confirmed. Curve (B) represents the exclusion region based on these considerations.

If the anomalous result seen in the contained neutrino events is not due to high mixing angle $\nu_{\mu}$ oscillation to $\nu_{r}$, then it is interesting to speculate on other possible causes:

1. The effect is a fluke (so Soudan II should not see it).

2. The effect is due to $\nu_{\mu} \rightarrow \nu_{\tau}$ oscillations in the region not excluded by the upward-going muon results. 


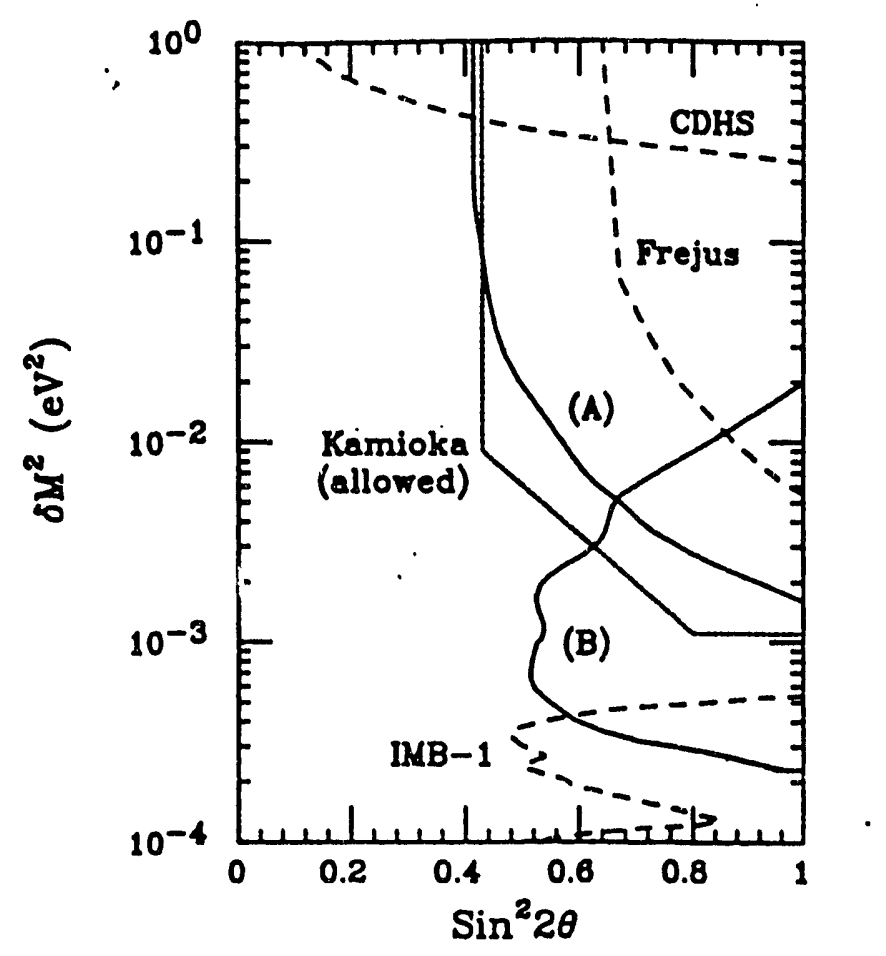

Figure 4: $90 \% \mathrm{cl}$ limits on $\nu_{\mu}$ to $\nu_{\tau}$ oscillations based on upward-going muons from IMB.

3. The effect is due to some previously unknown nuclear effects in neutrino interactions.

4. The effect is due to $\nu_{\mu}$ to $\nu_{e}$ oscillations.

5. The effect is due to astrophysical $\nu_{e}$ being injected into the atmospheric neutrino spectrum.

6. The effect is due to proton decay.

With the exception of the first possibility, the other possible reasons above are all quite intriguing. Thus there are now several ideas being discussed in the community for long-baseline neutrino oscillation experiments, the construction of very large water Cerenkov detectors, more precise measurements of the atmospheric pion and kaon flux, etc. For IMB, we will try and look at the possibility of $\nu_{\mu}$ to $\nu_{e}$ oscillations, proton decay, and an anomalous astrophysical $\nu_{e} f l u x$. Work on this is now in progress.

\section{c. IMB Works in Progress}

Now that data reduction from tape is almost complete, there are several other papers being now worked on. They include: 
1. An analysis of the full 7.7 kton-year IMB-3 contained data set for evidence of proton decay. This will be the "definitive" IMB nucleon decay paper.

2. A search for ultra-high energy neutrino interactions (discussed above).

3. A paper on IMB neutrino astronomy results, combining IMB-1,2, and -3 data. This will include a precise measurement of the flux of upward-going muons as a function of zenith angle.

4. A search for the possible stellar collapse origin of gamma ray bursts.

5. Testing of the contained data set for possible proton decay into single-prong electron final states.

It is expected (by us at, $\mathrm{JU}$, anyway) that these papers will all be completed by February, 1994. Unless we think of something else important later on, this should essentially complete the IMB data analysis (with the exception of the post-calibration data, which is discussed below).

\section{d. Joint IMB/Kamiokande Beam Test at KEK}

The neutrino flavor measurements of IMB and Kamiokande depend heavily on the ability of these detectors to separate muon-like events from electron-like events. Though this capability has been tested via the use of Monte Carlo simulations and of downward-going entering muons, there is always the worry that the Monte Carlo may not be accurate (to be polite), or systematic erros due to the fact that the geometry of downward going muons is certainly very different than the contained neutrino interactions. In addition to this, there may be systematic errors in $\mu / e$ separation which are not possible to measure in a realistic sense other than in a charged particle beam, where the energy, geometry, and particle type of the beam is under control.

Such a controlled experiment has now been approved as a joint collaboration of the IMB and Kamiokande groups. These tests will use a 1 kton model detector to be built at the KEK accelerator facility in Japan. The tank will be instrumented with both IMB and Kamiokande PMT's and electronics, approximating the original IMB light collection efficiency and geometry as closely as possible. A pion/muon/electron test beam will then be injected into the tank in several different places. The beam momentum will be adjusted in steps of $100 \mathrm{MeV} / \mathrm{c}$ from 100 to $2000 \mathrm{MeV} / \mathrm{c}$ for the separate particle types (as appropriate). These measurements will allow definitive 
subcontract (though funds past 31 January, 1993 are not officially approved yet from UCI).

\section{e. Superkamiokande}

There is still a considerable amount of interest in the scientific community over the scientific questions addressed by the Kamioka and IMB detectors, such as nucleon decay, solar neutrinos, and neutrino oscillations. This interest has led to the design of a new 32,000 ton (10 times IMB) water Cerenkov detector, SuperKamiokande, to be constructed in Japan. Both R.Svoboda and M.Cherry have joined the nowforming American contingent of this collaboration. Both are working on the joint IMB/Kamiokande beam tests described above.

Superkamiokande would expand the search for nucleon decay a factor of 10-30 beyond current limits. It would also allow higher statistics measurements of the atmospheric neutrino flux in order to arrive at a $\nu_{\mu}$ and $\nu_{e}$ spectrum which would better support statistical tests for neutrino oscillations. The American Superkamiokande proposal will be finished by November, 1992. No funds are requested for Superkamiokande for this year, but it is envisioned that as the IMB experiment winds down funds now being used at LSU for IMB can be dedicated to Superkamiokande.

\section{DUMAND}

LSU joined the DUMAND experiment in March, 1992 (R.Svoboda, and R.Clark, a graduate student). Since then a significant DUMAND construction effort has started here. The LSU effort is mainly in three areas, fabrication of titanium frames for optical modules, fabrication of the aluminum pressure housings for the underwater electronics, and organization of ihe off-line software group.

\section{a. DUMAND Current Status and Schedule}

The current schedule has the first DUMAND string in the water by early next summer, to be followed three months later by two more strings. This schedule appears to be holding firm, despite problems with the macro array chip being designed at Boston and the lag of the European Optical Modules. The chip design has been sent to the manufacturing company, and debugging of the design with the company is now underway. Funds to make European Optical modules now seem to be available, and the NSF has funded the DUMAND calibration system effort at Vanderbilt.

At the end of September, 1992 there will be a deployment practice cruise. This will be a run-through of the actual DUMAND deployment, including a $\frac{1}{4}$ size PMT string and a mock-up of the undersea junction box. The cruise will be aboard the USS Laney Chuest, which would be the vessel of choice for string deployment (with 
an AT\&T ship laying the cable from detector to shore). The practice deployment will include several surface to ocean floor dives by the deep submersible Sea Cliff to practice the string hook-up operation. The Laney Chuest is the mother ship for the Sea Cliff. Both are operated by the U.S. Navy.

Integration of the first string will take place at the University of Hawaii in January, 1993. A full string of 24 Optical Modules (OM's), Calibration Modules (CM's), and String Center Controller (SCC) will be assembled in a refrigerated van and plugged into a junction box. This operation will take about 6 weeks. From March until deployment time (June) the string will be operated as if it were deployed in the deep ocean. This will allow any final problems to present themselves and be dealt with. At the end of this testing period the decision will be made to go for deployment (barring serious problems).

While end-to-end testing is proceeding with the first string, assembly will begin on the next two strings. Their deployment will follow as soon after the initial string as possible, depending on assembly time and ship schedules. This will complete the first phase of DUMAND deployment and give us a $5,000 \mathrm{~m}^{2}$ detector, about 5 times the size of the full MACRO detector.

Over the next year, there will be four DUMAND meetings. Funds are requested for R.Svoboda to attend three of these meetings and for R.Clark to attend one. In addition, travel funds are also requested for three man-months of time at the DUMAND experimental site during the critical testing and deployment phase next spring and summer.

\section{b. DUMAND Titanium Frames}

The glass spheres that house the OM's and CM's hardly ever implode. If such an implosion were to occur in DUMAND, however, the potential damage could be quite severe if the implosion cut the string support cables, or fiber optic/power cables that run up the outside of the square optical module frame. To prevent this, tests were made in June 92 by intentionally imploding a sphere at depth with some prototype frames build of titanium at ISU. Though some welds on the frame parted, the string support members (the sections that hold the string together) remained intact. In addition, the titanium shields put between the sphere and the fiber/power lines were sucessful in keeping them from being damaged. Encouraged by this, two improved (based on test experience) versions of the titanium frames have been recently fabricated here and will be tested again on the September practice deployment. These tests will allow the design to be finalized.

Due to the success of the June tests, the decision has been made to go witi titanium frames to protect the detector against implosion disasters. The frames will be 
mass-produced at LSU, where there is a staff machinist familiar with working with titanium. For three strings (and a spare), 120 frames will be needed. The first 30 frames will be built with FY 91-92 funds (originally intended for IMB operations). In this proposal, we ask for funds for shop time to build the next 90 frames. Experience has shown that each frame takes about 6 hours to fabricate, and the shop charges $6 \$ /$ hour.

It should be noted that all the material costs associated with frame construction (titanium, special welding wire, titanium fasteners, etc) are being paid for by DU. MAND construction funds, not from the LSU operating budget.

\section{c. DUMAND Electronics Housings}

The String Center Controller (SCC) housings are also being manufactured at LSU. These consist of 7 ' long, 1' thick sections of extruded aluminum tube. These tubes have been delivered to LSU for machining of o-ring grooves, anodization, and manufacture of end plugs (which must pass fiber optic and electrical cables). Again, the first controller will be built out of FY 91-92 funds with the next three to be built out of 92-93 funds. Material costs will be borne by DUMAND construction funds. Each housing is estimated to take almost 70 hours of shop time to manufacture. The tubes are now at LSU, where they have been cut to the desired length. We are currently research the necessary anodizing specifications.

\section{d. DUMAND Off-Line Software}

R.Svoboda is head of the off-line DUMAND software group. He has been working on data format standards, and in writing the necessary calibration software. It is very important that sufficient off-line software be immediately available upon DU. MAND deployment in order to test detector operation while support ships are still engaged. Right now the main effort is in developing a real-time speed muon track fitting program for inclusion the the shore station software. No special funds are requested for this development above normal computer costs and regular travel funds to meetings. 


\section{E. SSC Activity}

\section{GENERAL}

Part of the LSU high energy group (Imlay, Metcalf, and Lyndon) and A. Fazely of Southern are participating on the SDC experiment. We have worked in the SDC calorimetry group on scintillation tile calorimetry, studying optical uniformity and radiation damage. This work started with an SSC detector subsystem proposal funded in 1990. Recently this work has been funded by DOE-SSC and by TNRL through the PDRC (Particle Detector Research Center at Prairie View). Presently LSU receives $\$ 38 \mathrm{~K}$ from the PDRC primarily to fund the postdoctoral C. Lyndon. This covers 9 months of his salary plus a little for travel and 3 months technician. The SDC decided in October ' 91 to proceed with the scintillator tile option and is preparing to build a prototype unit to be tested at Fermilab. We plan to participate in the Fermilab beam tests while continuing at a reduced level of optical studies at LSU.

C. Lyndon has worked with Professor McNeil on the GEM experiment as well as working on the SDC experiment. He has taken primary responsibility this year for overseeing and analyzing the tile test on uniformity and radiation damage. He also attends many of the SDC meetings and frequently presents LSU/Southern results. In addition he has worked a substantial fraction of his time with Professor McNeil on simulation studies for GEM and related beam studies at BNL and CERN. Lyndon has done good work on both projects.

We feel that it would be very desirable to have separate postdoctorals on GEM and SDC work. The travel required including work at accelerators makes it hard to meet the needs of both experiments. Both experiments would benefit substantially from having continuity provided by a full time physicist. Thus Roger McNeil is requesting funds in this proposal for a fulltime postdoctoral for GEM.

\section{SDC R \& D}

LSU was responsible for the production and testing of optical components for the electromagnetic portion of the ZEUS barrel calorimeter which uses scintillator-plate sampling. The optical response of components was measured with an $x-y$ scanning table in our laboratory. Our facilities for ZEUS production and testing, together with our experience made us ideally suited to perform optical studies to optimize this technology for the SSC. In addition a cobalt 60 source at the LSU Nuclear Science Center is very convenient for radiation damage studies and permits us to measure the optical response starting immediately after irradiation. 
In the summer of 1990 LSU developed its experimental test facility for measuring the optical performance of scintillation tiles. A collimated ruthenium beta source was mounted on our computer controlled X-Y scanning table. This setup allows us to measure the optical response of tiles. This test setup has been used for two years to measure the response of tiles with fiber readout. We have studied many tiles mostly supplied by Bicron for the effect of radiation on optical uniformity and absolute light yield (number of photoelectrons). We have also studied optical uniformity over the surface of the tile for various fiber readout patterns.

In appendix D is SDC note $92-172$ which describes our tile results as of late 1991 . These studies have continued. Fig. 18 shows recent results of radiation damage to a tile from SCSNS1. Studies of optical uniformity of large tiles $(-30 \mathrm{~cm} \times 30 \mathrm{~cm})$ are now in progress.

\section{GEM R \& D}

In 1991 McNeil joined the newly formed GEM Collaboration. He is member of the Muon Steering Committee and is coordinating the group's simulation efforts towards a successful muon system design. His work has been funded through the TNLRC.

The muon simulation effort at LSU can be divided into 5 main aspects

\section{The Total depth of calorimetry required}

The thickness of material before the calorimeter serves to filter hadrons and thus suppress the rate of hadron punchthrough. GEM's very small inner tracking volume already minimizes the backgrounds from decay-in-flight of pions and Kaons. McNeil has investigated the minimum calorimeter thickness required for the GEM detector. The results of the study is reported in the attached GEM Technical Note 92-69. McNeil also wrote parts of the responses to the PAC questions regarding the GEM LOI.

\section{A Fast Subroutine for Hadron Punchthrough}

Since 1989 McNeil has been at work to develop a fast simulation of hadron punchthrough showers. Despite advances in CPU power in recent years it is still impractical to utilize a Monte Carlo simulation (such as GEANT) which tracks all the secondaries in the hadron shower through 


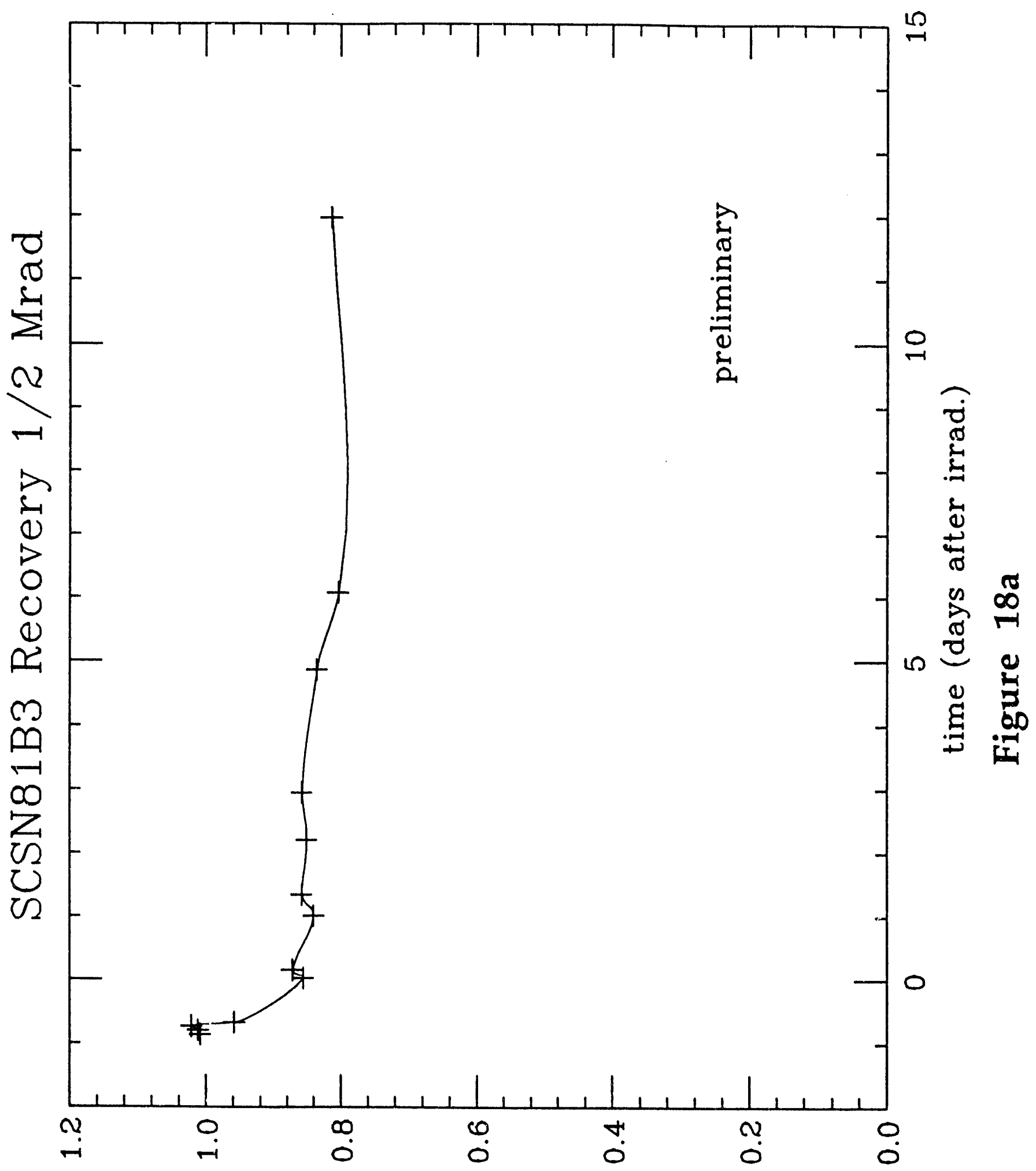

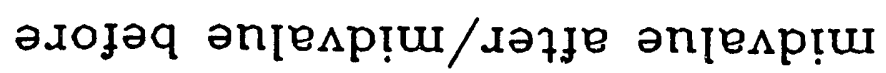




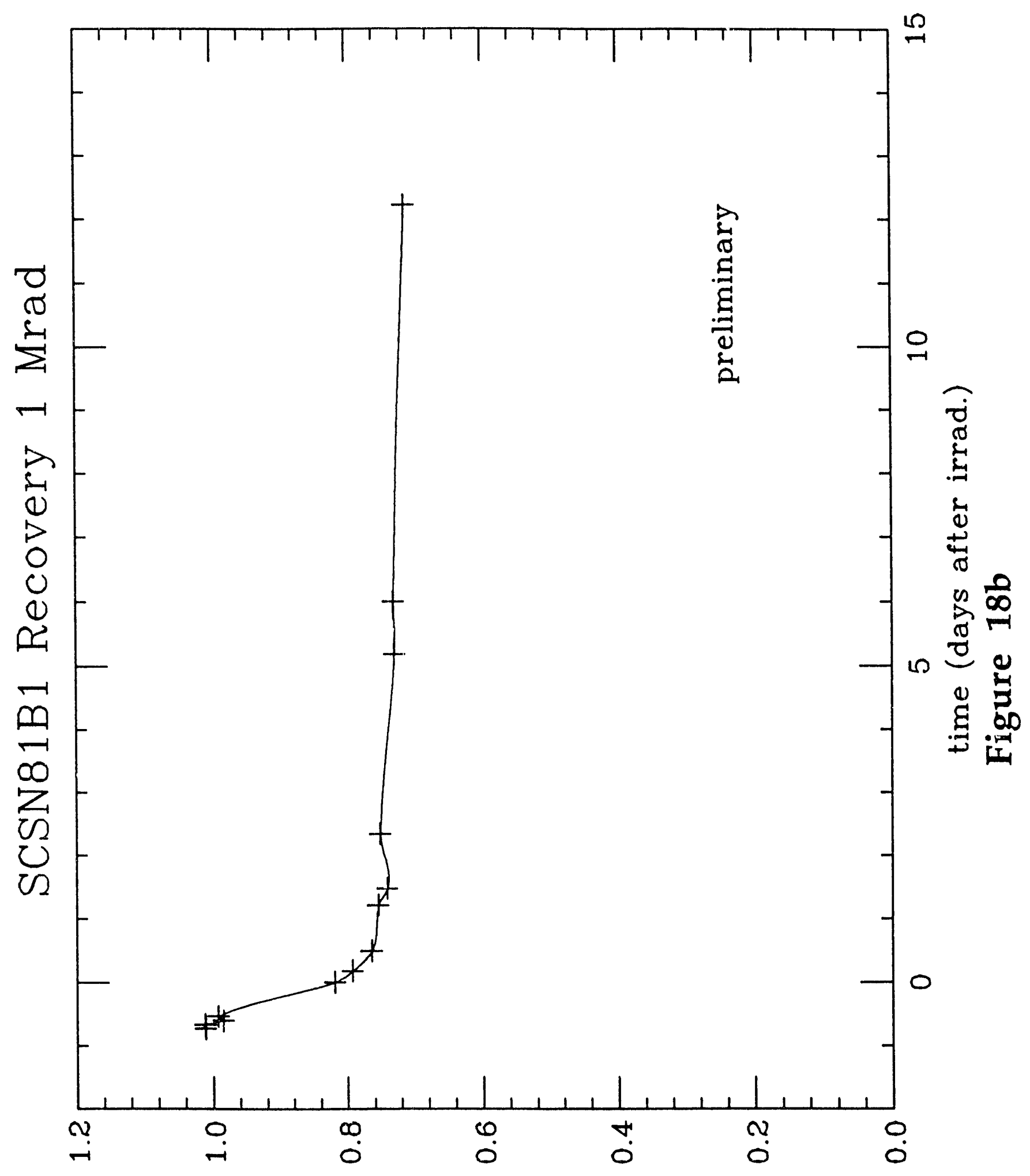

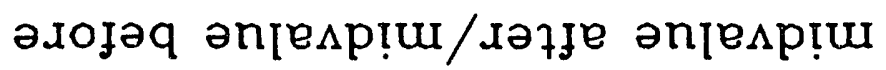




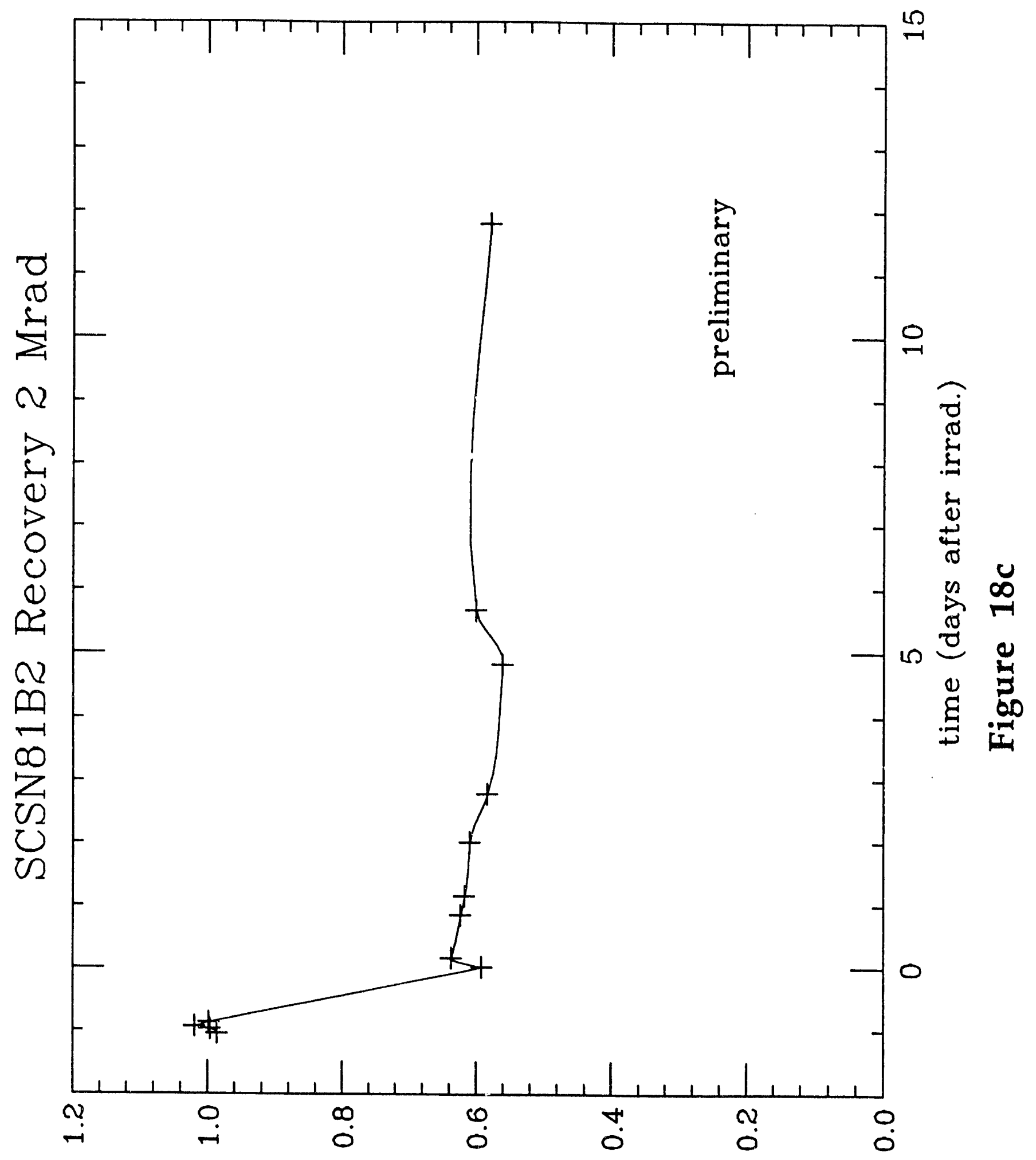

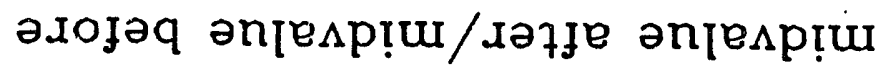


the calorimeter. McNeil has used GEANT simulation of single pions through material to prepare a set of probability tables. These tables contain enough information to produce outgoing 4-vectors of the charged punchthrough particles. The fast punchthrough simulation program known as PCHTHR has now been incorporated into both the GEM and SDC Muon Simulation efforts. This program will be useful in producing punchthrough showers in the muon system for triggering and pattern recognition studies. A copy of the transparencies of an invited talk delivered by McNeil at this past summer's Rocky Mountain Consortium Workshops is included as an appendix. A new version (V3.0) of PCHTHR which outputs the charged punchthrough particles as well as the gammas and neutrons is in preparation and should be released this fall.

\section{Muon Energy loss and Energy loss measurement}

The GEM Detector has special capabilities in the measurement of Gammas Electrons and Muons. The Muon measurement is made in air outside of the calorimetry in a field provided by a large 9 in. radius superconducting solenoid. The muon measurement is thus determined by the muon system momentum resolution and the affects of the muon energy loss (and energy loss measurement) through the calorimeter. If not measured in the calorimeter the fluctuations in the muon energy loss would enter into the muon measurement uncertainty as

$$
\left.\frac{\Delta \mathrm{P}}{\mathrm{P}}\right|_{\text {TOT }}=\left.\frac{\Delta \mathrm{P}}{\mathrm{P}}\right|_{\text {muon meas }}+\left.\frac{\Delta \mathrm{E}_{\text {loss }}}{\mathrm{P}}\right|_{\text {CAL }}
$$

McNeil has been performing simulation studies of muon energy loss to understand the affect and optimize the calorimeter material to minimize this contribution to the muon measurement uncertainty. A GEM Note $92-69$ of one study has been attached in the appendix.

McNeil is also participating in the beam tests of the copper plus scintillating fiber calorimetry, one of the technology options for the hadronic calorimeter being considered by GEM during this past summer. Chris Lyndon, a LSU post doc and Sam Khosravi, an LSU technician, went to ENL to assist in setting up the beam tests. Currently LSU is analyzing the muon beam data to compare the energy measurement with results from GEANT simulation.

\section{Muon induced Showers}

As energetic muons pass through materials they induce showers of electromagnetic particles. The accompanying particles can potentially obscure the muon position measurement and 
lead to degradation of the muon momentum resolution. The muon induced showers will become increasingly more common as the muon becomes more energetic and will be most severe in rare processes such as a high mass $Z^{\prime}$ decaying to 2 muons. McNeil has been simulating the showers induced by muons exiting the dense material of the calorimetry as well as the production of showers and delta rays as the muon passes through the material of the muon system itself, McNeil has been a member of the Limited Streamer Drift tube group within GEM. This group has tested a system of LSDT's in a beam of 0.5 Tev muons at FNAL during summer of 1991. McNeil performed preliminary GEANT simulations of the test beam setup and the agreement between data and GEANT was encouraging. Further comparisons are in progress and a NIM article is planned,

\section{Punchthrough measurements at CERN}

Recently, GEM collaboration members from LSU, SUNY at Stony Brook, Boston University and BNL have joined the RD5 Experiment at CERN. This effort has been funded by the TNLRC. The purpose of RD5 experiment is two-fold:

- To study the rate and characteristics of hadron punchthrough and muon induced showers at high energy. Hadron and muon beams of $300 \mathrm{Gev}$ are incident on a spectronmeter complete with magnetic field.

- To test different muon detector technologies in and determine their performance including rate capabilities.

LSU will be involved primarily in the punchthrough analysis. The goal will be to validate the GEANT simulation program in all aspects of punchthrough shower including the punchthrough probability, multiplicity of particles, momentum of particles, and exit angle of particles.

There will be three runs in the second half of 1992, in August, September and October. LSU post doc Chris Lyndon will travel to CERN for two months from early September and participate in the last two runs. He will assist GEM collaboration members in monitoring the performance of Cathode Strip Chambers being considered as a possible technology for the GEM Muon detector. He will also participate in the data taking and begin analysis of the punchthrough data. Simulations will begin at LSU where considerable resources are available. 
The work LSU performs for the GEM experiment is funded by the TNLRC which covers foreign and domestic travel, 3 months of post-doctoral salary, 3 months of Graduate Student salary (presently used to support of computer science graduate student to perform programming tasks), 1 month of technician salary, and about $\$ 10,000$ of undergraduate student salaries. To contribute more effectively to GEM we would need more physicist effort. It would be important to have a full time post doctoral researcher to take a significant role in the RD5 beam tests at CERN and BNL Beam Tests. It is therefore requested to have a DOE supported post doctoral researcher along with domestic travel funds to cover trips to collaboration meetings in the U.S. 


\section{F. References}

1. "New Limits on $\bar{v}_{\mu} \rightarrow \bar{v}_{e}$ Oscillation", L.S. Durkin, et al., Phys. Rev. Lett. 61, 1811 (1988).

2. "Limits on Neutrino Oscillations from $\bar{v}_{e}$ Appearance" S. Freedman, et al., to be published, Phys. Rev. D.

3. Muon neutrino carbon charged-current interaction near the Muon Threshold; D.D. Koetke, et al., LASL preprint LA-UR-92-1562 (submitted to Phys. Rev. C).

4. V. Zacek et al., Phys. Lett. 164B:193 (1985).

5. L.A. Ahrens et al., Phys. Rev. D31:2732 (1985).

6. T. Dombeck et al., Phys. Lett. 194B:591 (1987).

7. L. Borokovsky et al., Phys. Rev. Lett. 68, 274 (1992).

8. B. Bodmann et al., Phys. Lett. 267B, 321 (1991).

9. Cyrus M. Hoffman, Phys. Lett. 208B:149 (1988): Peter Herczeg, in "Proceedings of the Paris Workshop on Production and Decay of Light Mesons," ed. Patrick Fleury, World Scientific (1988), p.16; P. Herczeg and C.M. Hoffman, Phys. Lett. 100B:347 (1981); 102B:445(E) (1981).

10. L.A. Ahrens et al., Phys, Rev. D35:785 (1987).

David B. Kaplan and Aneesh Manohar, Nucl. Phys. B310:527 (1988).

11. N. Ushida et al., Phys. Rev. Lett. 57, 2897 (1986).

12. "Proceedings of the HERA Workshop", October 12-14, 1987, Hamburg, F.R. Germany, published by DESY and "Proceeding of the Workshop Physics at HERA", October 29-30, 1991, published by DESY.

13. "Proceedings of the Summer Study on High Energy Physics in the 1990's", June 27-July 15, 1988 Snowmass, Colorado, published by World Scientific Pub.

14. H. Tieke et al., Nucl. Inst. and Math., A277, 42 (1989). 
15. R. Becker-Szendy et al., Proc. of the 21st Int. Cosmic Ray Conf. v10, 6-19 January, Adelaide (1990).

16. F.W. Stecker et al., Phys. Rev. Lett. 66, 2697 (1991)

17. A.P. Szabo and R.J. Protheroe, Proc. of the High Energy Neutrino Astronomy Workshop, March 23-26, Honolulu (1992), to be published.

18. R. Becker-Szendy, et al., accepted by Phys. Rev D.

19. R. Becker-Szendy, et al., Phys. Rev. Lett. 69, 1010 (1992).

20. D. Casper, et al., Phys. Rev. Lett. 66, 2561 (1991).

21. H. Lee and Y.S. Koh, Nuovo Cimento B105, 883 (1990).

22. K.S. Hirata, et al., Phys. Lett. B205, 216 (1988). 


\section{REPORTS}

ORO-5490-1

ORO-5490-2

ORO-5490-3

ORO-5490-4

ORO-5490-5

ORO-5490-6

ORO-5490-7

ORO-5490-8

ORO-5490-9

ORO-5490-10

ORC-5490-11

ORO-5490-12

ORO-5490-13

ORO-5490-14

ORO-5490-15

ORO-5490-16
Comments on Electromagnetic Mass Differences in the Charmed Quark Model, Lai-Him Chan, LSU Preprint, 1977).

The Spin Structure of the Effective Quark Hamiltonian and the Hyperfine Splittings of Charmonium, Lai-Him Chan, Phys. Lett., $71 B, 422$ (1977).

Quark-Loop Dominance in the SU(3) Sigma Model, ,Lai-Him Chan, Phys. Rev. Lett., 39, 1124 (1977).

Charmonium - The 1P, State, Lai-Him Chan and R. F. O'Connell, Phys. Lett., 76B, 121 (1978).

Progress Report, March 1978.

The Gross-Neveu Model as a Z3 $=0$ Limit of the Two-Dimensional SU(N) Sigma Model, R. Haymaker and F. Cooper, Phys. Rev. DI9, 562 (1979).

Renormalization of the Broken Symmetry 2D SU(N) Sigma Model and the $\bar{\psi} \psi$ Model, R. Haymaker with F. Cooper,G. Guralnik, and K. Tamvakis, Phys. Rev. D20, 3336 (1974).

Progress Report, March 1979.

The Effect of Heavy Particle in Low Energy Light-Particle Processes, Lai-Him Chan, T. Hagiwara and B. Ovrut, Phys. Rev., D20, 982 (1979).

How to Measure the Number of Quark and Lepton Generations without Building Ultrahigh Energy Accelerators, Lai-Him Chan and T. Hagiwara, Phys. Rev. D20, 1968 (1979).

Progress Report, January 1980.

A Goldstone Pion with Bag Confinement, T. Goldman and R. Haymaker, LSU Preprint, (1980).

A Goldstone Pion with Bag Confinement, T. Goldman and R. Haymaker, Invited Talk Presented at the XX International Conference on High Energy Physics, Madison, Wisconsin, July 1980. (Published in the Proceedings, American Institute of Physics, 1981).

Dynamically Broken Chiral Symmetry with Bag Confinement, T. Goldman and R. Haymaker, Phys. Rev. D24, 724 (1981).

Bag Confinement Chiral Breaking Effect on a Goldstone Pion, T. Goldman and R. Haymaker, Phys. Lett. IOOB, 276 (1981).

Bag Boundary Condition for Confinement in the qq - Relative Coordinate, R. Haymaker and T. Goldman, Phys. Rev., D24, 743 
(1981).

ORO-5490-17

ORO-5490-18

ORO-5490-19

ORO-5490-20

ORO-5490-21

ORO-5490-22

ORO-5490-23

ORO-5490-24

DOE/ER/05490-25

DOE/ER/05490-26

DOE/ER/05490-27

DOE/ER/05490-28

DOE/ER/05490-29

DOE/ER/05490-30

DOE/ER/05490-31
Observation of the Upsilon" at the Cornell Electron Storage Ring, G. Finocchiaro et al. (including G. Levman, LSU) Phys. Rev. Lett., 45, 222 (1980).

Observation of the 'Transition $\mathrm{Y}^{\prime} \rightarrow \mathrm{Y}^{+} \pi^{-} \rightarrow \mathrm{e}^{+} \mathrm{e}^{-} \pi^{+} \pi^{-}, \mathrm{G}$. Mageras et al (Including R. Imlay and G. Levman, LSU) Phys. Rev. Lett. 46, 1115 (1981).

Hadron Electromagnetic Mass Differences and a Prediction of $\mathrm{B}^{+}$- B ${ }^{\circ}$ Lai-Him Chan, LSU Preprint, (1980).

Progress Report, February 1981.

Measurement of B-meson Semileptonic Decay, (including Imlay, Metcalf, Levman, Sreedhar, LSU), Phys. Rev. Lett. 47, 771 (1981).

Closed-Form Bound-State Effective Potential and Exact Solutions for Dynamical Symmetry Breaking in a 4D Gauge Theory, R.

Haymaker and J. Perez-Mercader, Phys. Lett. IO6B, 201 (1981).

Dynamical Symmetry Breaking. R. Haymaker, Lecture Notes for the XXI Cracow School of Theoretical Physics; Acta Physica Polonica B13, 575 (1982).

Progress Report, December 1981.

Dyriamics and Stability of Dynamical Symmetry Breaking without Fundamental Scalars, R. Haymaker and Juan Perez-Mercader, Phys. Rev. D27, 1353 (1983).

Dynamics of the Light Mesons Lai-Him Chan, LSU preprint, (1982).

Search for Structure in $\sigma\left(\mathrm{e}^{+} \mathrm{e}^{-} \rightarrow\right.$ hadrons) between $\sqrt{5}=10.34$ and $11.6 \mathrm{GeV}, \mathrm{E}$. Rice, et al.(including Imlay, Metcalf, Levman, and Sreedhar, LSU), Phys. Rev., Letters 48, 906 (1982).

Thrust Distributions and Decays of the $Y$ Bound States, D.

Peterson, T. Bohringer, et al. (including Imlay, Metcalf, Levman and Sreedhar, LSU), Phys. Letters, Ill4B, 277 (1982).

Upper Limit for Axion Production in Radiative Gamma Decay M. Sivertz, et al.(including Imlay, Metcalf, Levman and Sreedhar, LSU), Phys. Rev. D26, 717 (1982).

Search for B* $\rightarrow B+\gamma$ and limits on the B-meson mass, R. D. Schamberger, Jr., et al., (including Imlay, Metcalf, Levman, and Sreedhar, LSU), Phys. Rev., D26, 720 (1982).

Inclusive $K^{\circ}$ Production in $\mathrm{e}+\mathrm{e}-$ Annilhilation at the Upsilon 
Resonances, G. Giannini, et al, (including Imlay, Metcalf, Levman and Sreedhar, LSU), Nuclear Physics B206, 1 (1982).

DOE/ER/05490-32

DOE/ER/05490-33

DOE/ER/05490-34

DOE/ER/05490-35

DOE/ER/05490-36

DOE/ER/05490-37

DOE/ER/05490-38

DOE/ER/05490-39

DOE/ER/05490-40

DOE/ER/05490-41

DOE/ER/05490-42

DOE/ER/05490-43

DOE/ER/05490-44

DOE/ER/05490-45
Convexity of the Effective Potential, R. Haymaker and J. PerezMercader, Phys. Rev. D27, 1948 (1983).

Observation of $\pi \pi$ Cascade Decays of the Upslion" G. Mageras,et al. (including Imlay, Levman, Metcalf, and Sreedhar,LSU), Phys. Lett. I18B, 453 (1982).

Hadron Electromagnetic Mass Differences and a Prediction of B+$B^{\circ}$, I ai-Him Chan, LSU preprint. (Revision) Phys. Rev. Lett, 51, 253 (1983).

Observation of $\mathrm{P}$-wave $\mathrm{b} \overline{\mathrm{b}}$ - Bound States, K. Han, et al.,(including Imlay, Levman, Metcalf, and Sreedhar, LSU), Phys. Rev. Letters, 49, 1612 (1982).

Evidence for $\chi_{b}$ Production in the Exclusive Reaction $Y^{\prime \prime} \rightarrow \gamma \chi_{b}{ }^{\prime} \rightarrow\left(\gamma \gamma Y^{\prime}\right.$ or $\left.\gamma \gamma Y\right), G$. Eigen, et al., (including Imlay, Levman, Metcalf, and Sreedhar, LSU, Phys. Rev. Lett.,49, 1616 (1982).

Progress Report, December 1982.

Stability of a Chiral Breaking Vacuum, R. Haymaker and J. PerezMercader, preprint, to be published in the Proceedings of the Workshop on Non-Perturbative QCD, Oklahoma State University, 1983; Birkhauser.

Natural, Large, Dynamically Generated Gauge Hierarchies, R. Haymaker and J. Perez Mercader, LANL Preprint LA-UR-83-2332, (1983).

Observation of the Lowest P-Wave b $\bar{b}-$ Bound States, $C$.

Klopfenstein et al., (including Imlay, Metcalf, Levman, and Sreedhar) Phys. Rev. Lett., 51, 160 (1983).

Progress Report, October 1983

Tunneling and the Low Momentum Expansion of the Effective Action, C. Bender, F. Cooper, B. Freedman, R. Haymaker, Nucl Phys., B256, 653 (1985).

Anomalous $Z^{\circ}$ Events and Scalar Bound States, R. Haymaker and T. Matsuki, Phys. Lett. 148B, 243 (1984).

Association of Supersymmetry with $\mathrm{g}=2$ for the Electron, $\mathrm{R}$. Haymaker and A. R. P. Rau, LSU Preprint, (1985).

Isospin Mass Splittings of Hadrons with Heavy Quarks, Lai-Him Chan, Phys. Rev. D31, 204 (1985). 
DOE/ER/05490-46

DOE/ER/05490-47

DOE/ER/05490-48

DOE/ER/05490-49

DOE/ER/05490-50

DOE/ER/05490-51

DOE/ER/05490-52

DOE/ER/05490-53

DOE/ER/05490-54

DOE/ER/0\$490-55

DOE/ER/05490-56

DOE/ER/05490-57

DOE/ER/05490-58

DOE/ER/05490-59

DOE/ER/05490-60
Observation of $\chi_{b}$ Production in the Exclusive Reaction $\mathrm{Y}^{\prime} \rightarrow \gamma \chi_{b} \rightarrow \gamma \gamma \mathrm{Y} \rightarrow \gamma \gamma\left(\mathrm{e}^{+} \mathrm{e}^{-}\right.$or $\left.\mu^{+} \mu^{-}\right)$F. Pauss et al., (including Imlay, Levman, Metcalf, Sreedhar) Phys. Lett., I30B, 444 (1983).

Semileptonic Decay of the B Meson, C. Klopenstein, et al,.(including Imlay, Levman, Metcalf, Sreedhar), Phys. Lett., 130B, 44 (1983).

The CLEO Detector, D. Anders, et al.,(including Imlay) Nuclear Instru. and Meth., 2II, 47 (1983).

Observation of Direct photons in $Y$ and $Y^{\prime}$ Decays and Determination of the QCD Scale Parameter, R. D. Schamberger, et al., (including Han, Imlay, Levman, Metcalf, Sreedhar) Phys. Lett., 138B, 225 (1984).

Search for Higgs Scalars in Upsilon Decays, S. Youssef, et al., (including Han, Imlay, Levman, Metcalf, Sreedhar), Phys. Lett., I39B, 332 (1984).

A Measurement of the Semileptonic Decay of B Mesons into Muons, G. Levman, et al., (including Sreedhar, Han, Imlay, Metcalf), Phys. Lett., 141B, 271 (1984).

A Study of Hadronic Decays of the Y', V. Fonseca, et al., (including Imlay, Levman, Metcalf) Nucl. Phys., B242, 31 (1984).

Improved Method for Studying Tunneling and False Vacuum Decays, R. Haymaker, T. Matsuki, S. Wang, and F. Cooper, Abstract, submitted to AIP Santa Fe meeting DPF, Fall, 1984.

A New Approach to the Low Energy Effective Action Expansion, Lai-Him Chan, Abstract submitted to APS, DPF, Santa Fe meeting, Fall, 1984

Can Baryons be Chiral Solitons in the Effective Lagrangian of the Non-Linear SU(N) Quark Model? Lai-Him Chan, Abstract submitted to the APS DPF Santa Fe meeting Fall, 1984.

General Calculation of the N=4 Supersymmetric Yang-Mills Self Energy in an Effective Light-Cone Gauge Formalism, T. Matsuki, and G. Leibbrant, Phys. Rev., D31, 934 (1985).

Chiral and Graviational Anomalies in Any Dimension, R. Delbourgo and T. Matsuki, J. Math. Phys. 26, 1334 (1985).

Another Resonance observed in a Multi TeV e+e-Collider, T. Matsuki, LSU preprint, (1984).

New Limits on the Mass of Majorana Neutrino and Right-handed Heavy Bosons, A. Fazely, F. Imlay, T. Matsuki and W. Metcalf.

Progress Report October 1984. 
DOE/ER/05490-61

DOE/ER/05490-62

DOE/ER/05490-63

DOE/ER/05490-64

DOE/ER/05490-65

DOE/ER/05490-66

DOE/ER/05490-67

DOE/ER/05490-68

DOE/ER/05490-69

DOE/ER/05490-70

DOE/ER/05490-71

DOE/ER/05490-72

DOE/ER/05490-73

DOE/ER/05490-74

DOE/ER/05490-75

DOE/ER/05490-76

DOE/ER/05490-77
Effective-Action Expansion in Perturbation Theory, Lai-Him Chan, Phys. Rev. Lett., 54, 1222 (1985).

Baryons as Solitons in the Effective Lagrangian of Spontaneously Broken Chi al Symmetry, Lai-Him Chan, Phys. Rev. Lett., 55, 21 (1985).

Lorentz Anomaly in Arbitrary Dimensions, T. Matsuki, to be published in Zeit. f. Phys. C (1985).

Gravitational Counterterms and BRS Symmetry, R. Delbourgo and T. Matsuki, Phys. Rev. D32, 2579 (1985).

Quantum Corrections to False Vacuum Decay in the ColemanWeinberg Potential, F. Cooper, R.W. Haymaker, T. Matsuki, and S. Wang, Phys. Rev. D32, 2049 (1985)

Gravitational Counterterms in an Axial Gaizge, T. Matsuki, Phys. Kev. D32, 3164 (1985).

Effective Lagrangian for the Linear $\sigma$ Model at $\mathbf{M}_{\sigma} \rightarrow \infty$, Lai-Him Chan, LSU Preprint (1985).

PC Database for High Energy Preprint Collections, R. W. Haymaker, LSU Preprint (1985).

Saddle Point Instability in Models of Chiral Symmetry Breaking, $\mathbf{R}$. W. Haymaker and T. Matsuki,Phys Rev., D33, 1137 (1986).

Supersymmetry in Quantum Mechanics, R. W. Haymaker and A. R. P. Rau, Am. J. Phys., 54, 928 (1985).

Path Integral Derviation of Gravitational Anomalies in Arbitrary Even Dimensions, T. Matsuki, Progrs. Theor. Phys., 75, 461 (1986).

Masses, Widths and Leptonic Widths of the Higher Upsilon Resonances, D. M. J. Lovelock et al, Phys. Rev. Lett., 54, 377 (1985).

Observation of B* Production in e+e- Interactions abnve the bFlavor Threshold, K. Han et al.,Phys. Lett, 55, 36 (1985).

Progress Report, September 1985.

Comparison of Alternative Effective Potentials for Dynamical Symmetry Breaking, R. W. Haymaker, T. Matsuki, and F. Cooper, Phys. Rev. D35, 2567 (1987).

Derivative Expansion for the one-loop effective actions with Internal Symmetry, L-H Chan, Phys. Rev. Lett, 57, 1199 (1986)

Is the Nonlinear $\mathbf{M}_{\sigma} \rightarrow \infty$ Limit of the Linear $\sigma$ Model? L-H Chan, Phys. Rev. D36, 3755 (1987). 
DOE/ER/05490-78

DOE/ER/05490-79

DOE/ER/05490-80

DOE/ER/05490-81

DOE/ER/05490-82

DOE/ER/05490-83

DOE/ER/05490-84

DOE/ER/05490-85

DOE/ER/05490-86

DOE/ER/05490-87

DOE/ER/054P0-88

DOE/ER/05490-89

DOE/ER/05490-90

DOE/ER/05490-91

DOE/ER/05490-92

DOE/ER/05490-93

DOE/ER/05490-94
Effective Lagrangian for the Chiral Quark Dynamics, L-H Chan, Chiral Soliton, Chapter 2, 64-69, edited by K. F. Lin (World Scientific, Singapore, 1986)

Neutrinoless Double $\beta$ decay and its Relation to Pion Double Charge Exchange, A. Fazely and L. C. Liu, LS-UR-86-447 (1986), Phys. Rev. Let: 57, 968 (1986)

Progress Report, September 1986

Space Structure of Confining Strings, J. Wosiek and R. W. Haymaker, Phys. Rev. D36, 3297 (1987)

Complex Langevin Simulations of Nonabelian Integrals, R. W. Haymaker and J. Wosiek, Phys. Rev. D37, 969 (1988).

Improvement of the Derivative Expansion, Lai-Him Chan, Phys. Rev. D38, 3739 (1988).

Progress Report, October 1987

Complex Langevin Simulation of Non-Abelian Flux Integrals, R. W. Haymaker and J. Wosiek, Nuclear Physics B4, 571-575, (1988).

Hierarchies under the Rainbow, R. W. Haymaker and J. PerezMercader, LSU preprint, (1988).

Measurements of R and a Search for Heavy-Quark Production in e+e- Annihilation at $\sqrt{\mathrm{s}}=50$ and $52 \mathrm{GeV}, \mathrm{H}$. Sagawa, et al., Phys. Rev. Lett. 60, 93-96 (1988).

Search for Isolated Leptons in Low-Thrust e+e- Annihilation events at $\sqrt{\mathrm{S}}=50$ and $52 \mathrm{GeV}$, S. Igarashi, et al., Phys. Rev. Lett. 60, 2359-2362 (1988).

Experimental Mass Limit for a Fourth-Generation Sequential Lepton from e+e- Annihilations at $\sqrt{\mathrm{s}}=56 \mathrm{GeV}, \mathrm{G}$. N. Kim, et al., Phys. Rev. Lett. 61, 911-914 (1988).

Recent Results from AMY at TRISTAN, R. R. McNeil, KEK prepri:: $87-90$ (1987).

Nonanalog 0+(g.s.) $\rightarrow$ 0+(g.s.) Transitions in Pion Double Charge Exchange Reactions on 128Te and 130 Te, A. Fazely, et al., Phys. Letters B208, 361-364 (1988).

Effective Action Expansion for the Non-Linear Sigma Model: The Normal Coordinate Method, L.-H. Chan, W. F. Kao, and T. Matsuki, LSU preprint (1988).

Progress Report, October 1988

Convergence of Langevin Simulation for Complex Gaussian Integrals, R. W. Haymaker and Y Peng, Phys. Rev. D41, 1269 (1990). 
DOE/ER/05490-95

DOE/ER/05490-96

DOE/ER/05490-97

DOE/ER/05490-98

DOE/ER/05490-99

DOE/ER/05490-100

DOE/ER/05490-101

DOE/ER/05490-102

DOE/ER/05490-103

DOE/ER/05490-104

DOE/ER/05490-105

DOE/ER/05490-106
Color Flux Distribution on the Lattice, R. W. Haymaker, presented at the XXIX Cracow School of Theoretical Physics, Zakopane, Poland, June 1989.

Limits on $\overline{v_{\mu}} \rightarrow \overline{v_{e}}$ Oscillations, L. S. Durkin, et al., Phys. Rev. Lett. 61, 1811 (1988).

Physics from TRISTAN, invited address at the Conference on "Beyond the Standard Model," R. R. McNeil, Iowa State University, Ames Iowa, November 1988, Proceedings to be pubished by Academic Press.

Measurements of Cross Sections and Charge Asymmetries for e+e$\rightarrow \tau^{+} \tau^{-}$and $\mathrm{e}^{+} \mathrm{e}^{-} \rightarrow \mu^{+} \mu-$ for $\sqrt{\mathrm{s}}$ from 52 to $57 \mathrm{GeV}$., A. Bacala, et al., Phys. Lett. B218, 112 (1989).

Measurements of the e+e- Total Hadronic Cross Section and a Determination of $\mathrm{Mz}$ and $\Lambda \overline{\mathrm{MS}}$, T. Mori, et al., Phys. Lett. B218, 499 (1989).

Experimental Evidence for the non-Aiclian Nature of QCD from a Study of Multijet Events Produced in e+e- Annihilations, I. H.

Park, et al., Phys. Rev. Lett., 62, 1713 (1989).

A Fine-Grain Modular Detector for Low-Energy Particles, L. S. Durkin, et al., Nucl. Instrum. Method A277, 386 (1989).

Search for the Substructure of Leptons in High Energy QED Processes at TRISTAN, S. K. Kim, et al., Phys. Lett. B223, 476 (1989).

"Recent Results from TRISTAN, W. J. Metcalf, invited talk at the 2nd International Conference on the 4th Family of Quarks and Leptons, UCLA, Febrruary 23-25, 1989.

Search for Unstable Heavy Neutral Leptons in $\mathrm{e}^{+} \mathrm{e}-$ Annhilations at $\sqrt{\mathrm{s}}$ from 50 to $60.8 \mathrm{GeV}$, N. M. Shaw, et al., Phys. Rev. Lett., 63, 1342 (1989).

Consequence on Scale Invariance, H. Cheng and W. F. Kao, LSU HE preprint, (1989).

${ }^{12} \mathrm{C}\left(\pi^{ \pm}, \pi^{ \pm^{\prime}}, \mathrm{p}\right){ }^{11} \mathrm{~B}$ Reaction near the Giant Dipole Region, S. H. 
Yoo, et al., Phys. Rev. Lett., 63, 738 (1989)

DOE/ER/05490-107

DOE/ER/05490-108

DOE/ER/05490-109

DOE/ER/05490-110

DOE/ER/05490-111

DOE/ER/05490-112

DOE/ER/05490-113

DOE/ER/05490-114

DOE/ER/05490-115

DOE/ER/05490-116

DOE/ER/05490-117

DOE/ER/05490-118

DOE/ER/05490-119
Progress Report, October 1989.

Variational Methods for Composite Operators: R. Haymaker, Reviste del Nuovo Cimento 14 no. 7 (1991).

Color Flux Distribution on the Lattice: R. W. Haymaker and J. Wosiek, Acta Physica Polonica, B21, 403 (1990).

High Precision Study of the Space Distribution of the Colour Flux in SU(2) Lattice Gauge Theory: R. W. Haymaker, Y. Peng, V. Singh, and J. Wosiek, Proceedings of the International Workshop Lattice '89, Capri, Italy, Nucl. Phys. B (Proc. Suppl.) 17, 558 (1990).

Convergence of Langevin Simulation for Complex Gaussian Integrals: R. Haymaker and Y. Peng, Proceedings of the DPF90 meeting at Rice University, January 1990, World Scientific (1990) P 791.

Color Flux Distribution in Pure SU (2) Lattice Gauge Theory: R. W. Haymker, .Y. Peng, V. Singh, and J. Wosiek, Proceedings of the DPF90 meeting at Rice University, January 1990, World Scientific (1990) P 789.

Search for non-minimal Higgs Production in $\mathrm{e}^{+} \mathrm{e}^{-}$Annihilations a $v \bar{s}=56$ GeV., E. H. Low, et al., Phys. Lett, B 228, 548 (1989).

Comparison of quark and gluon jets produced in high energy $\mathrm{e}^{+} \mathrm{e}^{-}$ Annihilations, Y. K. Kim, et al., Phys. Rev. Lett, 631772 (1989).

Search for a Fourth-Generation Charge-1/3 Quark, S. Eno et al., Phys. Rev. Lett., 63, 1910 (1989).

Measurement of $\mathrm{e}^{+} \mathrm{e}^{-} \rightarrow \mathrm{b} \overline{\mathrm{b}}$ forward-backward change asymmetry between $\sqrt{\mathrm{s}}=52$ and $57 \mathrm{GeV}, \mathrm{H}$. Sagawa, et al., Phys. Rev. Lett., 63, 2341 (1989).

Forward-Backward Charge Asymmetry in $\mathrm{e}^{+} \mathrm{e}^{-} \rightarrow$ Hadron Jets, D. Stuart et al., Phys. Rev. Lett., 64, 983 (1990).

A Search for Leptoquark and Colored Lepton Pair Production in e+e- Annihilations at Tristan, G. N. Kim, et al., Phys. Lett. B, 240, 243 (1990).

Multihadron-event properties in $\mathrm{e}^{+} \mathrm{e}^{-}$Annihilation at $\sqrt{\mathrm{s}}=52-57$ 
GeV, Y. K. Li, et al., Phys. Rev. D., 412675 (1990).

DOE/ER/05490-120

DOE/ER/05490-12i

DOE/ER/05490-122

DOE/ER/05490-123

DOE/ER/05490-124

DOE/ER/40617-125

DOE/ER/40617-126

DOE/ER/40617-127

DOE/ER/40617-128

DOE/ER/40617-129

DOE/ER/40617-130
Observation of Anomalous Production of Muon Pairs in $\mathrm{e}^{+} \mathrm{e}^{-}$ Annihilation into Four-Lepton Final States, Y. H. Ho, et al., Phys. Lett. B, 244, 573 (1990).

Charged Hadron Multiplicities in $\mathrm{e}^{+} \mathrm{e}^{-}$Annihilations at $\sqrt{\mathrm{s}}=50$ to 61.4 GeV, H. W. Zheng, et al., Phys. Rev. D, 42, 737 (1990).

Mass Limits of Charged Higgs at Large tan $(\beta)$ from $\mathrm{e}^{+} \mathrm{e}^{-}$ Annihilations at $\sqrt{\varsigma}=50-60.8 \mathrm{GeV}, \mathrm{J}$. R. Smith, et al., Phys. Rev. D, 42,949 (1990).

Progress Report, September 1990.

Distribution of the Color Fields Around Static Charges: Lattice Sum Rules, with J. Wosiek, Phys. Rev. D, 43, 2676 (1991).

Energy and Action of the Colour Field Around Static Quarks (with V. Singh and J. Wosiek), Proceedings of the International Conference on Lattice Field Theory, Tallahassee, Nucl. Phys. B. (Proc. Suppl.) 20, 207 (1991).

Measurement of Atmospheric Neutrino Composition with the IMB-3 Detector, D. Casper et al., Physical Review Letters, 66, 2561 (1991).

Progress Report, September 1991.

London Relation and Fluxoid Quantization for Monopole Currents in U(1) Lattice Guage Theory, V. Singh, R. Haymaker and D. Browne, LSU Preprint LSUHEP-1-92, May, 1992.

Vacuum Structure of Pure Gauge Theories on the Lattice R. Haymaker, V. Singh, D. Browne and J. Wosiek, LSU Preprint LSUHEP\#130-1992.

Introduction to Lattice Guagefixing and Effective Quark and Gluon Masses, C. Bernard, A. Soni and K. Yee, to be published in the Proceedings of the Workshop on QCD Vacuum Structure, Paris, France, 1992. 
DOE/ER/40617-131 Search for Cygnus X-3 in Underground Muons During the 1989 Radio Outbursts Using the IMB Detector, R. Becker-Szendy et al., Phys. Rev. D, 43, 1413 (1991).

DOE/ER/40617-132 Search for Muon Neutrino Oscillations with the Irvine-MichiganBrookhaven Detector, R. Becker-Szendy et al., Physical Review Letters, 69, 1010 (1992).

DOE/ER/40617-133 The IMB-3 Detector, R. Becker-Szendy, et al., accepted by NIM June 19, 1992.

DOE/ER/40617-134 Progress Repor1, September 1992. 

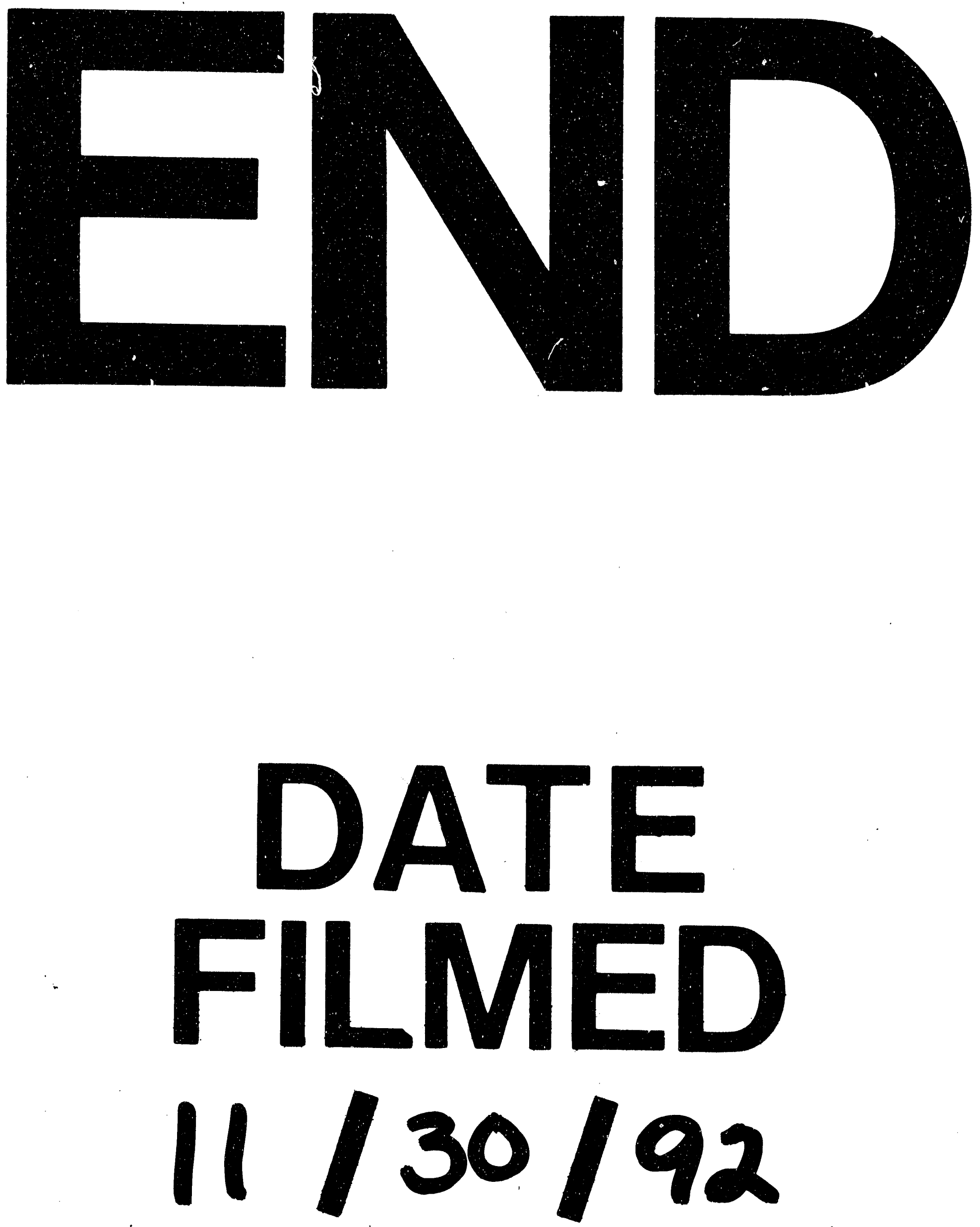
